\title{
Analysis and quantification of the diversities of aerosol life cycles within AeroCom
}

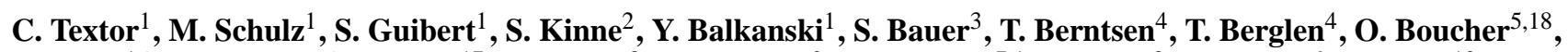
M. Chin ${ }^{16}$, F. Dentener ${ }^{6}$, T. Diehl ${ }^{17}$, R. Easter ${ }^{8}$, H. Feichter ${ }^{2}$, D. Fillmore ${ }^{7,1}$, S. Ghan ${ }^{8}$, P. Ginoux ${ }^{9}$, S. Gong ${ }^{10}$,

A. Grini ${ }^{4}$, J. Hendricks ${ }^{11}$, L. Horowitz ${ }^{9}$, P. Huang ${ }^{10}$, I. Isaksen ${ }^{4}$, T. Iversen ${ }^{4}$, S. Kloster ${ }^{2}$, D. Koch ${ }^{3}$, A. Kirkevåg ${ }^{4}$, J. E. Kristjansson ${ }^{4}$, M. Krol ${ }^{12}$, A. Lauer ${ }^{11}$, J. F. Lamarque ${ }^{7}$, X. Liu ${ }^{13}$, V. Montanaro ${ }^{14}$, G. Myhre ${ }^{4}$, J. Penner ${ }^{13}$,

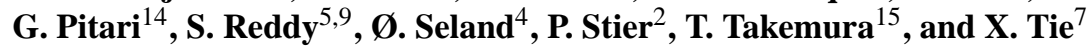

${ }^{1}$ Laboratoire des Sciences du Climat et de l'Environnement, Gif-sur-Yvette, France

${ }^{2}$ Max-Planck-Institut für Meteorologie, Hamburg, Germany

${ }^{3}$ Columbia University, GISS, New York, USA

${ }^{4}$ University of Oslo, Department of Geophysics, Oslo, Norway

${ }^{5}$ Laboratoire d'Optique Atmosphérique, Université des Sciences et Technologies de Lille, CNRS, Villeneuve d'Ascq, France

${ }^{6} \mathrm{EC}$, Joint Research Centre, Institute for Environment and Sustainability, Climate Change Unit, Italy

${ }^{7} \mathrm{NCAR}$, Boulder, Colorado, USA

${ }^{8}$ Battelle, Pacific Northwest National Laboratory, Richland, USA

${ }^{9}$ NOAA, Geophysical Fluid Dynamics Laboratory, Princeton, New Jersey, USA

${ }^{10}$ ARQM Meteorological Service Canda, Toronto, Canada

${ }^{11}$ Institut für Physik der Atmosphäre, DLR Oberpfaffenhofen, Germany

${ }^{12}$ Institute for Marine and Atmospheric Research Utrecht (IMAU) Utrecht University, Utrecht, Netherlands

${ }^{13}$ University of Michigan, Ann Arbor, MI, USA

${ }^{14}$ Universita degli Studi L'Aquila, Italy

${ }^{15}$ Kyushu University, Fukuoka, Japan

${ }^{16}$ NASA Goddard Space Flight Center, Greenbelt, MD, USA

${ }^{17}$ Goddard Earth Sciences and Technology Center, University of Maryland Baltimore County, Baltimore, Maryland, USA

${ }^{18}$ Hadley Centre, Met Office, Exeter, UK

Received: 31 May 2005 - Published in Atmos. Chem. Phys. Discuss.: 9 September 2005

Revised: 29 November 2005 - Accepted: 13 December 2005 - Published: 29 May 2006

\begin{abstract}
Simulation results of global aerosol models have been assembled in the framework of the AeroCom intercomparison exercise. In this paper, we analyze the life cycles of dust, sea salt, sulfate, black carbon and particulate organic matter as simulated by sixteen global aerosol models. The differences among the results (model diversities) for sources and sinks, burdens, particle sizes, water uptakes, and spatial dispersals have been established. These diversities have large consequences for the calculated radiative forcing and the aerosol concentrations at the surface. Processes and parameters are identified which deserve further research.
\end{abstract}

The AeroCom all-models-average emissions are dominated by the mass of sea salt (SS), followed by dust (DU), sulfate $\left(\mathrm{SO}_{4}\right)$, particulate organic matter (POM), and finally black carbon (BC). Interactive parameterizations of the emissions and contrasting particles sizes of SS and DU lead gen-

Correspondence to: $\mathrm{C}$. Textor

(christiane.textor@cea.fr) erally to higher diversities of these species, and for total aerosol. The lower diversity of the emissions of the fine aerosols, $\mathrm{BC}, \mathrm{POM}$, and $\mathrm{SO}_{4}$, is due to the use of similar emission inventories, and does therefore not necessarily indicate a better understanding of their sources. The diversity of $\mathrm{SO}_{4}$-sources is mainly caused by the disagreement on depositional loss of precursor gases and on chemical production. The diversities of the emissions are passed on to the burdens, but the latter are also strongly affected by the model-specific treatments of transport and aerosol processes. The burdens of dry masses decrease from largest to smallest: DU, $\mathrm{SS}, \mathrm{SO}_{4}$, $\mathrm{POM}$, and $\mathrm{BC}$.

The all-models-average residence time is shortest for SS with about half a day, followed by $\mathrm{SO}_{4}$ and DU with four days, and POM and $\mathrm{BC}$ with six and seven days, respectively. The wet deposition rate is controlled by the solubility and increases from DU, BC, $\mathrm{POM}$ to $\mathrm{SO}_{4}$ and $\mathrm{SS}$. It is the dominant sink for $\mathrm{SO}_{4}, \mathrm{BC}$, and $\mathrm{POM}$, and contributes about one third to the total removal of SS and DU species. For SS

Published by Copernicus GmbH on behalf of the European Geosciences Union. 
and DU we find high diversities for the removal rate coefficients and deposition pathways. Models do neither agree on the split between wet and dry deposition, nor on that between sedimentation and other dry deposition processes. We diagnose an extremely high diversity for the uptake of ambient water vapor that influences the particle size and thus the sink rate coefficients. Furthermore, we find little agreement among the model results for the partitioning of wet removal into scavenging by convective and stratiform rain.

Large differences exist for aerosol dispersal both in the vertical and in the horizontal direction. In some models, a minimum of total aerosol concentration is simulated at the surface. Aerosol dispersal is most pronounced for $\mathrm{SO}_{4}$ and $\mathrm{BC}$ and lowest for SS. Diversities are higher for meridional than for vertical dispersal, they are similar for the individual species and highest for SS and DU. For these two components we do not find a correlation between vertical and meridional aerosol dispersal. In addition the degree of dispersals of SS and DU is not related to their residence times. $\mathrm{SO}_{4}, \mathrm{BC}$, and $\mathrm{POM}$, however, show increased meridional dispersal in models with larger vertical dispersal, and dispersal is larger for longer simulated residence times.

\section{Introduction}

Atmospheric aerosols play a key role in many important environmental issues including climate change, stratospheric ozone depletion and tropospheric air pollution. Aerosols are significant components within the global climate system as they absorb and scatter solar and terrestrial radiation. However, the aerosol radiative forcing is not well quantified. Global-scale models that simulate the processes of emission, dispersion, chemical and physical transformations, removal, and radiative properties in the troposphere play a central role in assessing the climate impact of aerosols and their gaseous precursors. The AeroCom initiative was created in 2003 to provide a platform for detailed evaluations of aerosol simulation in global models. Various complex aerosol models have been developed in recent years, but they have not been compared to each other in a consistent way. Even a detailed literature survey can hardly reveal and quantify all differences among existent models. The analysis of current global aerosol simulations based on harmonized diagnostics is the aim of the aerosol model intercomparison initiative AeroCom (http://nansen.ipsl.jussieu.fr/AEROCOM). The approach of AeroCom is twofold: The performance of global aerosol models is evaluated by intercomparisons with each other and by comparisons to observations of aerosol properties and processes. Such an analysis leads to understanding of differences so that uncertain components can be identified and the simulated aerosols properties can be improved. This work is a major extension of what was already achieved in Penner et al. (2001, 2002); Kinne et al. (2003). The models cited in these studies have evolved considerably and deserve reevaluation. Within AeroCom the diagnostics have been greatly extended and allow now for the analysis of aerosol life cycles in the different models, because information about sources and sinks, particle sizes, aerosol water, and others are included.

All global aerosol models taking part in this study have been carefully validated when the model authors compared them to various high-quality observational data sets. These included in-situ measurements of aerosol concentration, size distribution, and chemical composition, lidar measurements of the vertical distribution of aerosol extinction coefficient, sun photometer measurements of aerosol optical depth and column size distribution, and satellite measurements of the spatial distribution of aerosol optical depth. Please refer to the literature about the models cited in Table 2. However, observations are not free of uncertainties, and the results from the models differ considerably despite careful validation. We cannot identify a "best model" with respect to all data. Model performances change from parameter to parameter, and from region to region. In addition, the quantities, which are compared to observations, result from many interdependent properties and processes that are internally simulated by the models.

In this paper, the aerosol life cycles as simulated by sixteen global aerosol models are analyzed in order to explain the differences in the simulated aerosol fields. The quantification of model diversities facilitates identifying weak components where research is needed in order to improve our understanding of global atmospheric aerosol. It is not the objective of this work to judge the different ways of modeling the aerosol life cycle. This would necessitate investigating individual processes (e.g., water uptake) or concepts (e.g., representation of the size distributions). As these are strongly interrelated, several full sets of sensitivity simulations with strong constraints on all components except for the one under investigation should be performed. However, such studies are not feasible in the context of a volunteer based model intercomparison such as AeroCom, and because of the large differences of the participating models.

The coarse resolution of global models in space and time requires the parameterizations of many aerosol processes. These cannot always be well constrained, because observations on the micro-scale are lacking. Therefore some parameterizations might only be valid in the context of a specific model environment and under certain conditions, and not necessarily reflect the process for which they are intended. Models might produce erroneous results especially under changed conditions such as climate change scenarios. Disagreement among models about individual aerosol processes as quantified in this study helps to identify parameterizations of individual aerosol processes that require improvement.

AeroCom focuses on the five most important aerosol components (dust, sea salt, sulfate, black carbon and particulate organic matter), even if some models include 
other species. In the remainder of this paper we use the following component abbreviations: $\mathrm{DU}=$ mineral dust, $\mathrm{SS}=$ sea salt, $\mathrm{SO}_{4}=$ sulfate, $\mathrm{BC}=$ black carbon, and $\mathrm{POM}=$ particle organic matter, AER=total dry aerosol, $\mathrm{H} 2 \mathrm{O}=$ water, AmbAER=ambient aerosol. The simulations have been performed with the models in their usual configuration (AeroCom experiment A). The results of comparisons of models with observational data and those from the second AeroCom experiment $\mathrm{B}$ with identical aerosol emission fluxes and particle size distributions (Dentener et al., 2006) are discussed in follow-up papers (Guibert et al., 2006 ${ }^{1}$; Kinne et al., 2006; Schulz et al., $2006^{2}$; Textor et al., $2006^{3}$ ).

In the next section we give an overview of the models participating in AeroCom and the aerosol modules embedded therein. Then we describe our concept of quantifying the diversity (disagreement) of the model components. We compare the simulated aerosol sources and burdens, and the tropospheric residence times in Sects. 4, 5 and 6, respectively. In the following Sect. 7, the comparison focuses on the analysis of the individual removal processes based on the global rate coefficients for sedimentation, other dry and wet deposition. The microphysical properties involved, particle size, composition and uptake of ambient water, are discussed in Sect. 8. Section 9 deals with the spatial distributions and discusses the relationship with the removal rate coefficients. The paper closes with a summary and conclusions.

\footnotetext{
${ }^{1}$ Guibert, S., Schulz, M., Kinne, S., Textor, C., Balkanski, Y., Bauer, S., Berntsen, T., Berglen, T., Boucher, O., Chin, M., Dentener, F., Diehl, T., Feichter, H., Fillmore, D., Ghan, S., Ginoux, P., Gong, S., Grini, A., Hendricks, J., Horowitz, L., Isaksen, I., Iversen, T., Kloster, S., Koch, D., Kirkevåg, A., Kristjansson, J. E., Krol, M., Lauer, A., Lamarque, J. F., Liu, X., Montanaro, V., Myhre, G., Penner, J., Pitari, G., Reddy, S., Seland, Ø., Stier, P., Takemura, T., and Tie, X.: Global aerosol models tested against surface observations within AeroCom, in preparation, 2006.

${ }^{2}$ Schulz, M., Kinne, S., Guibert, S., Textor, C., Balkanski, Y., Bauer, S., Berntsen, T., Berglen, T., Boucher, O., Chin, M., Dentener, F., Diehl, T., Feichter, H., Fillmore, D., Ghan, S., Ginoux, P., Gong, S., Grini, A., Hendricks, J., Horowitz, L., Isaksen, I., Iversen, T., Kloster, S., Koch, D., Kirkevåg, A., Kristjansson, J. E., Krol, M., Lauer, A. Lamarque, J. F., Liu, X., Montanaro, V., Myhre, G., Penner, J., Pitari, G., Reddy, S., Seland, Ø., Stier, P., Takemura, T., and Tie, X.: Radiative forcing by aerosols as derived from the AeroCom present-day and pre-industrial simulations, in preparation, 2006.

${ }^{3}$ Textor, C., Schulz, M., Guibert, S., Kinne, S., Balkanski, Y., Bauer, S. E., Berntsen, T., Berglen, T., Boucher, O., Fillmore, D., Ginoux, P., Gong, S., Grini, A., Hendricks, J., Horowitz, L., Huang, P., Isaksen, I., Iversen, T., Koch, D., Kirkevåg, A., Kristjansson, J. E., Lauer, A., Lamarque, J. F., Liu, X., Montanaro, V., Myhre, G., Penner, J., Pitari, G., Reddy, S., Seland, Ø., Takemura, T., and Tie, $\mathrm{X}$.: The effect of harmonized emissions in AeroCom experiment B on aerosol properties in global models, in preparation, 2006.
}

Table 1. Abbreviations used in the tables.

\begin{tabular}{ll}
\hline Abbreviation & Signification \\
\hline accm & accumulation mode \\
act & activated \\
aitm & aitken mode \\
anthrop & anthropogenic \\
bioburn & biomass burning \\
cf & cloud free \\
coag & coagulation \\
coam & coarse mode \\
coeff & coefficient \\
cond & condensation \\
conv & convective \\
dyn & dynamics \\
emi & emission \\
ext & external \\
fix & prescribed \\
fraction & value in cloud free fraction of grid box \\
hetero & heterogeneous \\
homo & homogeneous \\
insol & insoluble \\
int & internal \\
interstit & interstitial \\
mean & mean grid box value \\
mix & mixture \\
modal M & modal scheme, mass mixing \\
modal MN & ratio as prognostic variable \\
modal scheme, mass mixing ratio and \\
nucl & number conc. as prognostic variables \\
nuclm & nucleation \\
NVOC & nucleation \\
prod & non-volatile organic carbons \\
prog & production \\
scav & prognostic \\
sigma-p & scavenging \\
sol & hybrid-sigma p \\
strat & soluble \\
supcoam & stratiform \\
super coarse mode \\
\hline & thermodynamics \\
\hline
\end{tabular}

\section{Description of the models}

Sixteen global aerosol models are currently taking part in the AeroCom model intercomparison, see Table 2. The aerosol modules are implemented in global "driver-models", which provide information on the meteorology, the surface conditions, etc. The simulated aerosol fields are the result of the combined and interdependent effects of the internal aerosol processes and of the aerosol transport provided by the driver models, which depend on the simulated meteorology. For example, the emissions of DU and SS depend on the wind fields, and the surface properties determine dry deposition rate coefficients. The parameterization 
Table 2. Description of the driver-models and general classification of aerosol modules.

\begin{tabular}{|c|c|c|c|c|c|c|c|c|c|}
\hline Model & $\begin{array}{r}\text { Global } \\
\text { model }\end{array}$ & $\begin{array}{l}\text { Horizont. } \\
\text { Resolution } \\
\text { (\#lon \#lat) } \\
\text { (lon lat) }\end{array}$ & $\begin{array}{l}\text { Vertical } \\
\text { Resolution } \\
\text { (\# levels) } \\
\text { (type) }\end{array}$ & $\begin{array}{l}\text { References for } \\
\text { global model }\end{array}$ & $\begin{array}{l}\text { Type of } \\
\text { scheme }\end{array}$ & $\begin{array}{l}\text { Number of } \\
\text { bins or modes }\end{array}$ & $\begin{array}{l}\text { Aerosol } \\
\text { mixing }\end{array}$ & $\begin{array}{l}\text { Aerosol } \\
\text { dynamics* }\end{array}$ & $\begin{array}{l}\text { References for } \\
\text { aerosol module }\end{array}$ \\
\hline ARQM & $\begin{array}{l}\text { GCM } \\
\text { Canadian GCMIII }\end{array}$ & $\begin{array}{l}128 \times 64 \\
2.81^{\circ} \times 2.81^{\circ}\end{array}$ & $\begin{array}{l}32 \\
\text { sigma-p }\end{array}$ & $\begin{array}{l}\text { Zhang and } \\
\text { McFarlane (1995b) }\end{array}$ & bin & $\begin{array}{l}12 \\
\text { all internally mixed }\end{array}$ & int & $\begin{array}{l}\text { nucl, coag, cond, } \\
\text { thermodyn, } \\
\text { cloud processing }\end{array}$ & $\begin{array}{l}\text { Zhang et al. (2001); } \\
\text { Gong et al. ( 2003) }\end{array}$ \\
\hline DLR & $\begin{array}{l}\text { GCM } \\
\text { ECHAM4 }\end{array}$ & $\begin{array}{l}96 \times 48 \\
3.75^{\circ} \times 3.75^{\circ}\end{array}$ & $\begin{array}{l}19 \\
\text { sigma }\end{array}$ & $\begin{array}{l}\text { Roeckner et al. } \\
\text { (1996) }\end{array}$ & $\begin{array}{l}\text { modal } \\
\text { MN }\end{array}$ & $\begin{array}{l}2 \\
\text { nuclm+accm }\end{array}$ & int & $\begin{array}{l}\text { nucl, cond, coag, } \\
\text { thermodyn, } \\
\text { aging BC POM }\end{array}$ & Ackermann et al. (1998) \\
\hline GISS & $\begin{array}{l}\text { GCM } \\
\text { modelE }\end{array}$ & $\begin{array}{l}46 \times 72 \\
5^{\circ} \times 4^{\circ}\end{array}$ & $\begin{array}{l}20 \\
\text { sigma }\end{array}$ & $\begin{array}{l}\text { Schmidt et al. } \\
\text { (2006) }\end{array}$ & bin & $\begin{array}{l}13 \\
2 \mathrm{SS}, 4 \mathrm{DU}, 1 \mathrm{BC}, \\
1 \mathrm{POM}, \\
1 \mathrm{SO}_{4}, \\
4 \mathrm{DU} \mathrm{SO}_{4}\end{array}$ & ext & $\begin{array}{l}\text { aging BC POM, } \\
\text { hetero DU-SO }\end{array}$ & $\begin{array}{l}\text { Koch et al. (1999, 2006); } \\
\text { Koch (2001); Bauer and } \\
\text { Koch (2006); ; Cakmur et } \\
\text { al. (2006); Koch and } \\
\text { Hansen (2005); Miller et } \\
\text { al. (2006) }\end{array}$ \\
\hline GOCART & $\begin{array}{l}\text { CTM } \\
\text { GOCART 3.15b }\end{array}$ & $\begin{array}{l}144 \times 91 \\
2.5^{\circ} \times 2.0^{\circ}\end{array}$ & $\begin{array}{l}30 \\
\text { sigma }\end{array}$ & $\begin{array}{l}\text { Atlas and Lucchesi } \\
(2000)\end{array}$ & modal M & $\begin{array}{l}17 \\
8 \mathrm{DU}, 4 \mathrm{SS}, 2 \mathrm{BC}, \\
2 \mathrm{POM}, 1 \mathrm{SO}_{4}\end{array}$ & ext & aging BC POM & $\begin{array}{l}\text { Chin et al. }(2000,2002) \text {; } \\
\text { Ginoux et al. (2001) }\end{array}$ \\
\hline $\begin{array}{l}\text { KYU } \\
\text { (SPRINT) }\end{array}$ & $\begin{array}{l}\text { GCM } \\
\text { CCSR/NIES/FRCGC } \\
\text { GCM / SPRINTARS } \\
5.7 \mathrm{~b}\end{array}$ & $\begin{array}{l}320 \times 160 \\
1.1^{\circ} \times 1.1^{\circ}\end{array}$ & $\begin{array}{l}20 \\
\text { sigma }\end{array}$ & $\begin{array}{l}\text { Numaguti et al. } \\
\text { (1995); Hasumi and } \\
\text { Emori (2004) }\end{array}$ & $\begin{array}{l}\text { bin, } \\
\text { modal M } \\
\text { for } \\
\text { aerosol } \\
\text { dyn }^{*+}\end{array}$ & $\begin{array}{l}17 \text { bins } \\
10 \mathrm{DU}, 4 \mathrm{SS}, 1 \mathrm{BC}, \\
1 \mathrm{BCPOM}, 1 \mathrm{SO}_{4} \\
\text { ( } 5 \text { modes for aerosol } \\
\text { dyn) }\end{array}$ & $\begin{array}{l}\text { ext } \\
\text { partly int for } \\
\text { BC/ POM }\end{array}$ & none & $\begin{array}{l}\text { Takemura et al. }(2000, \\
\text { 2002, 2005) }\end{array}$ \\
\hline LSCE & $\begin{array}{l}\text { GCM } \\
\text { LMDzT } 3.3\end{array}$ & $\begin{array}{l}96 \times 72 \\
3.75^{\circ} \times 2.5^{\circ}\end{array}$ & $\begin{array}{l}19 \\
\text { sigma }\end{array}$ & $\begin{array}{l}\text { Sadourny and Laval } \\
\text { (1984); Hourdin and } \\
\text { Armengaud (1999) }\end{array}$ & $\begin{array}{l}\text { modal } \\
\text { MN }\end{array}$ & $\begin{array}{l}5 \\
\text { accm: sol+insol, } \\
\text { coam: sol+insol, } \\
\text { supcoam: sol }\end{array}$ & $\begin{array}{l}\text { ext mix } \\
\text { of int } \\
\text { modes }^{3}\end{array}$ & aging BC POM & $\begin{array}{l}\text { Claquin et al. (1998, } \\
\text { 1999); Guelle et al. (1998a, } \\
\text { b, 2000); Smith and } \\
\text { Harrison (1998); } \\
\text { Balkanski et al. (2003); } \\
\text { Bauer et al. (2004); Schulz } \\
\text { et al. (2006) }\end{array}$ \\
\hline LOA & $\begin{array}{l}\text { GCM } \\
\text { LMDzT } 3.3\end{array}$ & $\begin{array}{l}96 \times 72 \\
3.75^{\circ} \times 2.5^{\circ}\end{array}$ & $\begin{array}{l}19 \\
\text { sigma }\end{array}$ & $\begin{array}{l}\text { Sadourny and Laval } \\
\text { (1984); Hourdin and } \\
\text { Armengaud (1999) }\end{array}$ & bin & $\begin{array}{l}16 \\
2 \mathrm{DU}, 11 \mathrm{SS}, \\
1 \mathrm{BC}(\text { sol+insol), } \\
1 \mathrm{POM} \text { (sol+insol), } \\
1 \mathrm{SO}_{4}\end{array}$ & ext & aging BC POM & $\begin{array}{l}\text { Boucher and Anderson } \\
\text { (1995); Boucher et al. } \\
\text { (2002); Reddy and } \\
\text { Boucher (2004); Guibert et } \\
\text { al. (2005) }\end{array}$ \\
\hline MATCH & $\begin{array}{l}\text { CTM } \\
\text { MATCH v } 4.2\end{array}$ & $\begin{array}{l}192 \times 94 \\
1.9^{\circ} \times 1.9^{\circ}\end{array}$ & $\begin{array}{l}28 \\
\text { sigma-p }\end{array}$ & $\begin{array}{l}\text { Zhang and } \\
\text { McFarlane (1995a); } \\
\text { Rasch et al. (1997); } \\
\text { Rasch and } \\
\text { Kristjansson (1998) }\end{array}$ & bin & $\begin{array}{l}8 \\
4 \mathrm{DU}, 1 \mathrm{SS}, 1 \mathrm{BC}, \\
1 \mathrm{POM}, 1 \mathrm{SO}_{4}\end{array}$ & ext & aging BC POM & $\begin{array}{l}\text { Barth et al. (2000); Rasch } \\
\text { et al. }(2000,2001)\end{array}$ \\
\hline MPI_HAM & $\begin{array}{l}\text { GCM } \\
\text { ECHAM5 }\end{array}$ & $\begin{array}{l}192 \times 96 \\
1.8^{\circ} \times 1.8^{\circ}\end{array}$ & $\begin{array}{l}31 \\
\text { sigma-p }\end{array}$ & $\begin{array}{l}\text { Roeckner et al. } \\
\text { (2003, 2004); Stier } \\
\text { et al. (2005) }\end{array}$ & $\begin{array}{l}\text { modal } \\
\text { MN }\end{array}$ & 7 & $\begin{array}{l}\text { ext mix of } \\
\text { int modes }\end{array}$ & $\begin{array}{l}\text { nucl, cond, coag, } \\
\text { thermodyn }\end{array}$ & Stier et al. (2005) \\
\hline MOZGN & $\begin{array}{l}\text { CTM } \\
\text { MOZART v2.5 }\end{array}$ & $\begin{array}{l}192 \times 96 \\
1.9^{\circ} \times 1.9^{\circ}\end{array}$ & $\begin{array}{l}28 \\
\text { sigma-p }\end{array}$ & $\begin{array}{l}\text { Brasseur et al. } \\
\text { (1998); Tie et al. } \\
\text { (2001, 2005); } \\
\text { Horowitz et al. } \\
\text { (2003) }\end{array}$ & bin & $\begin{array}{l}12 \\
1 \mathrm{SO}_{4}, 1 \mathrm{POM}, \\
1 \mathrm{BC}, 5 \mathrm{DU}, 4 \mathrm{SS}\end{array}$ & ext & aging BC POM & Tie et al. $(2001,2005)$ \\
\hline PNNL & $\begin{array}{l}\text { GCM } \\
\text { MIRAGE 2/ derived } \\
\text { from NCAR } \\
\text { CAM2.0 }\end{array}$ & $\begin{array}{l}144 \times 91 \\
2.5^{\circ} \times 2.0^{\circ}\end{array}$ & $\begin{array}{l}24 \\
\text { sigma-p }\end{array}$ & $\begin{array}{l}\text { Kiehl and Gent } \\
\text { (2004) }\end{array}$ & $\begin{array}{l}\text { modal } \\
\text { MN }\end{array}$ & $\begin{array}{l}8 \\
\text { aitm accm } \\
\text { coam DU+SO } \\
\mathrm{SS}_{4}+\mathrm{SO}_{4} \text {, coam } \\
\text { interstit+act each }\end{array}$ & $\begin{array}{l}\text { ext mix of } \\
\text { int modes }\end{array}$ & $\begin{array}{l}\text { nucl, cond, coag, } \\
\text { thermodyn, } \\
\text { cloud processing }\end{array}$ & Easter et al. (2004) \\
\hline TM5 & $\begin{array}{l}\text { CTM } \\
\text { TM5 }\end{array}$ & $\begin{array}{l}\text { global } 60 \times 45 \\
6^{\circ} \times 4^{\circ} \\
\text { Europe+North } \\
\text { America: } \\
1^{\circ} \times 1^{\circ}\end{array}$ & $\begin{array}{l}25 \\
\text { sigma-p }\end{array}$ & Krol et al. (2005) & $\begin{array}{l}\text { modal } \\
\text { MN }\end{array}$ & $\begin{array}{l}8 \\
3 \mathrm{SS}, 2 \mathrm{DU}, \\
1 \mathrm{SOA}-\mathrm{POM}, 1 \mathrm{BC}, \\
1 \mathrm{SO}_{4}-\mathrm{NO}_{3}\end{array}$ & ext & aging $B C$ & Metzger et al. (2002a, b) \\
\hline UIO_CTM & $\begin{array}{l}\text { CTM } \\
\text { OsloCTM2 }\end{array}$ & $\begin{array}{l}128 \times 64 \\
2.81^{\circ} \times 2.81^{\circ}\end{array}$ & $\begin{array}{l}40 \\
\text { sigma }\end{array}$ & Berglen et al. (2004) & bin & $\begin{array}{l}25 \\
8 \mathrm{DU}, 8 \mathrm{SS}, \\
4 \mathrm{BC}, 4 \mathrm{POM}, 1 \mathrm{SO}_{4}\end{array}$ & $\begin{array}{l}\text { ext } \\
\text { except } \\
\text { bioburn }\end{array}$ & aging BC POM & $\begin{array}{l}\text { Grini et al. (2002b, 2005); } \\
\text { Myhre et al. (2003); } \\
\text { Berglen et al. (2004); } \\
\text { Berntsen et al. (2006) }\end{array}$ \\
\hline UIO_GCM & $\begin{array}{l}\text { GCM } \\
\text { CCM3.2 }\end{array}$ & $\begin{array}{l}128 \times 64 \\
2.81^{\circ} \times 2.81^{\circ}\end{array}$ & $\begin{array}{l}18 \\
\text { sigma-p }\end{array}$ & $\begin{array}{l}\text { Hack (1994); Kiehl } \\
\text { et al. (1998) }\end{array}$ & $\begin{array}{l}\text { modal, } \\
\text { M/MN } \\
\text { bin for } \\
\text { aerosol } \\
\text { dyn }^{*+}\end{array}$ & $\begin{array}{l}12 \text { modes } \\
\text { aerosol dyn: } \\
43 \text { bins from } \\
8 \text { int modes } \\
\text { DU }+ \text { SS fix }\end{array}$ & $\begin{array}{l}4 \text { ext } \\
8 \text { int: mixed } \\
\text { from } 4 \text { prog } \\
+8 \text { fix }^{* * * * *}\end{array}$ & $\begin{array}{l}\text { nucl, cond, coag, } \\
\text { thermodyn, } \\
\text { cloud processing }\end{array}$ & $\begin{array}{l}\text { Iversen and Seland (2002); } \\
\text { Kirkevåg and Iversen } \\
\text { (2002); Kirkevåg et al. } \\
\text { (2005) }\end{array}$ \\
\hline ULAQ & $\begin{array}{l}\text { CTM } \\
\text { ULAQ }\end{array}$ & $\begin{array}{l}16 \times 19 \\
22.5^{\circ} \times 10^{\circ}\end{array}$ & $\begin{array}{l}26 \\
\log -p\end{array}$ & Pitari et al. (2002) & bin & $\begin{array}{l}41 \\
7 \mathrm{DU}, 9 \mathrm{SS}, 5 \mathrm{BC} \text {, } \\
5 \mathrm{POM}, 15 \mathrm{SO}_{4}\end{array}$ & ext & $\begin{array}{l}\text { aging BC POM } \\
\text { (Koch, 2001), } \\
\text { SO }_{4} \text { microphysics }\end{array}$ & Pitari et al. $(1993,2002)$ \\
\hline UMI & $\begin{array}{l}\text { CTM } \\
\text { IMPACT }\end{array}$ & $\begin{array}{l}144 \times 91 \\
2.5^{\circ} \times 2^{\circ}\end{array}$ & $\begin{array}{l}30 \\
\text { sigma-p }\end{array}$ & $\begin{array}{l}\text { Schubert et al. } \\
\text { (1993); Rotman et } \\
\text { al. (2004) }\end{array}$ & bin & $\begin{array}{l}13 \\
3 \mathrm{SO}_{4}, 1 \mathrm{POM}, \\
1 \mathrm{BC}, 4 \mathrm{DU}, 4 \mathrm{SS}\end{array}$ & ext & none & Liu and Penner (2002) \\
\hline
\end{tabular}

*) Aerosol dynamics refers to microphysical processes apart from those concerning the sulfur cycle, which is referenced in Table 5 .

**) KYU describes the size distributions differently for transport and aerosol dynamics. 16 bins (10 DU, $4 \mathrm{SS}, 1 \mathrm{BCPOM}, 1 \mathrm{SO} 4$ ) are considered for transport. For the aerosol dynamics, a modal approach is employed (one mode per species, sigma fix).

***) ext mix of int modes: Several internally mixed modes, which do not have the same composition and are therefore externally mixed.

****) UIO_GCM describes the size distributions differently for transport and aerosol dynamics. 12 modes are considered for transport, 4 of them have prescribed size distributions, are transported and not mixed with the other modes (external). The next 4 modes are also transported and only the shape of the distribution is constant (sigma fix). For the aerosol dynamics, these latter 4 modes are internally mixed with 8 prescribed modes, and fitted to 43 bins.

4) Schulz, M., Balkanski, Y., Textor, C., Guibert, S., Generoso, S., Boucher, O., Breon, F.-M., Hauglustaine, D., and Hourdin, F.: The LMDzT-INCA global aerosol model and its evaluation with surface, lidar and satellite aerosol observations, in preparation, 2006.

5) Bauer, S. E. and Koch, D.: Impact of Heterogeneous Sulfate Formation at Mineral Dust Aerosol Surfaces on Aerosol Loads and Radiative Forcing in the GISS GCM, J. Geophys. Res. A., submitted, 2006. 
of the hydrological cycle has a large influence; see Table 8 for references on the cloud microphysical parameterizations employed by the participating models. The local relative humidity governs hygroscopic particle growth, and the abundance of hydrometeors controls aerosol wash-out processes, although some aerosol modules use prescribed liquid water (or ice) content. In addition, the model results are influenced by rather technical aspects like the models' architectures and resolutions. The AeroCom intercomparison does not intend to investigate the global driver-models. However, as they strongly affect the aerosol life cycles, we briefly introduce their basic properties.

\subsection{Global models}

The references for the global driver-models can be found in Table 2. They are either chemical transport models (CTMs), or general circulation models (GCMs). CTMs calculate the aerosol distribution off-line based on prescribed meteorological data, which stem either from climate model simulations, or from analyzed weather observation systems. In GCMs, the aerosol transport is predicted on-line depending on the models' internal meteorology. The application of nudging techniques to GCMs allows them to closely represent observed weather patterns. Nudging signifies a Newtonian relaxation technique when an additional term is added to the model equations at each time-step to force them to a specific weather period (Jeuken et al. 1996). About half of the models participating in AeroCom are GCMs (ARQM, DLR, GISS, KYU, LSCE, LOA, MPI_HAM, PNNL, UIO_GCM) and the others are CTMs (GOCART, MATCH, MOZGN, TM5, UIO_CTM, ULAQ, UMI). Twelve of the models use analyzed meteorological observations and simulate specifically the year 2000 , while four models use climatological mean data (ARQM, ULAQ, UIO_GCM, DLR). The individual techniques employed to describe advection, convective transport, and turbulent mixing can be found in the literature cited in Table 2. The spatial resolutions of the participating models are highly varying, ranging from $1.1^{\circ} \times 1.1^{\circ}(51200$ grid points) to $22.5^{\circ} \times 10^{\circ}$ (304 grid points) in the horizontal, and from 18 to 40 layers in the vertical, see Table 2 .

\subsection{Aerosol modules}

The aerosol masses, compositions and size distributions, and the internal aerosol processes are described within specific aerosol modules that are implemented in the global models, see also Table 2. Atmospheric aerosol consists of a number of size modes that result from different production and loss processes. Several approaches are employed to describe the particle size distributions in numerical models (e.g., Seinfeld and Pandis, 1997). The simplest cases are the so-called bulk schemes, where the sizes of the aerosol particles are constant, and only the aerosol mass is predicted. In modal schemes, the particle size distribution is represented by mathematical functions, e.g., log-normal functions. The comprehensiveness of this type increases with the number of moments of the mathematical functions (number, mass, and width of the distribution) that are treated as prognostic variables, and with the number of modes. Most modern modal schemes consider two moments, aerosol mass and number concentrations, as prognostic variables, but use a fixed distribution width. In the third type, called bin (or spectral) schemes, the aerosol size distribution is represented by several size intervals. The accuracy, but also the computational costs, increase with the number of bins for which the aerosol mass is predicted.

Aerosol particles contain different components depending on their specific sources and their fate within the atmosphere. Field studies indicate both internal (all particles in a size class or mode have the same mixed composition) and external (i.e., each particle class or mode is composed of a single species) mixing. In general, primary particles are externally mixed close to their sources, but become internally mixed through coagulating with other particles or by condensation of gases on their surfaces. In the AeroCom models, the composition is described as external in eight models. In most others, aerosols are considered to be internally mixed within a mode, but externally mixed with other modes. For example, the fine mode is often described as an external mixture of a soluble and an insoluble mode, but each of these modes is an internal mixture of different chemical species. Furthermore, the aerosol modules describe the sources of aerosols and their removal processes. Most models distinguish between three removal pathways: wet deposition, dry deposition, and sedimentation. Note that hereafter dry deposition refers to surface removal due to turbulent transport, Brownian diffusion and impaction, excluding the contribution by sedimentation which is generally considered separately in the model analysis. Sources are discussed in Sect. 4, including the specific methods for the treatment of chemical $\mathrm{SO}_{4}$-production. The concepts for the sources and removal mechanisms as employed by the AeroCom models are examined in Sects. 4 and 7, respectively. Aerosol microphysical processes (also called aerosol dynamics) are only considered in some models (see Table 2). ARQM, DLR, MPI_HAM, PNNL, and UIO_GCM simulate nucleation, condensation, and coagulation of aerosols. Some models consider the formation of $\mathrm{SO}_{4}$ particles, but most include no microphysics except for aging (increase of solubility through oxidation) of $\mathrm{BC}$ and POM by transferring them from a hydrophobic to hydrophilic class using a fixed rate constant. Water uptake is represented in all models applying various parameterizations of different complexity, ranging from very simple approaches to more complex ones considering hysteresis effects or the activity of multicomponent aerosols, see Table 9. For a discussion of the simulated aerosol water content see Sect. 8.2. Models with interactive sulfur cycle simulate the aqueous phase oxidation of $\mathrm{SO}_{2}$ that leads to particle growth (see Table 5). Some include additional processing of aerosol particles by clouds (ARQM, MPI_HAM, PNNL, and 
UIO_GCM). Several others consider the effect of aerosols on clouds (indirect effect) (ARQM, PNNL, and UIO_GCM).

Table 2 provides an overview of the aerosol modules. Tables 2 to 9 give the references for the most important parameterizations and data sources employed in the aerosol modules taking part in AeroCom. Within the context of this study, we can neither distinguish the effects of the different approaches to describe the size distribution and mixing of aerosols, nor those of the complexity of individual parameterizations of aerosol processes. We rather focus on the analysis of aerosol fields, removal processes and selected microphysical properties that influence the aerosol life cycles.

\section{Methodology}

This paper synthesizes the information from sixteen global aerosol models compiled within the AeroCom exercise. We focus on the processes and properties that affect the simulated aerosol life cycles, and quantify the diversities among the models. Global, annual average properties are examined in this paper. These result from the parameterizations describing the aerosol processes under many different conditions and can therefore serve as indicators for the overall effects of the models' internal structure on the simulated aerosol fields. The model outputs were provided on their original grids. Global averages have been obtained with an area weighting. For simplicity we omit in the following notations like "global", "annual", and "annually averaged". Interpolation procedures were applied to obtain averages for subgrid volumes, e.g. height intervals or horizontal regions. As mentioned above, our results refer to the year 2000, except for the four climatological models. A conversion factor of 1.3 has been used to convert POM from the mass of organic carbon to dry organic mass if not otherwise indicated by the modelers, and POM is always given as dry organic mass. $\mathrm{SO}_{4}$ is considered as mass of $\mathrm{SO}_{4}$, except otherwise indicated. AER denotes total dry aerosol mass, i.e., the sum of the five aerosol species included in this study. Total aerosol is examined here because many observations refer to bulk properties, such as mass and volume measurements, or aerosol optical depth.

In the remainder of this paper, we use for simplification the term "anthropogenic" as an abbreviation to summarize $\mathrm{SO}_{4}, \mathrm{BC}$, and POM, although these species also have nonanthropogenic sources. SS and DU are denoted with the expression "natural", according to their main sources. The terms "ambient" or "wet" aerosol describe AER+H2O.

\subsection{The AeroCom data base}

Model results were assembled following an output specification protocol, and graphic visualizations of the data are published on the AeroCom web site. Modelers were asked to submit daily and monthly fields from their original model version. Resubmissions were permitted to correct for obvious model or data-analysis errors. Post-processing including simple calculations to complete the data sets was performed at the Laboratoire des Sciences du Climat et de l'Environnement in France. Detailed information was provided to the modelers on the AeroCom data web interface to communicate errors. Further tables and two-dimensional fields of all the properties discussed in this paper are available on the AeroCom web interface (http://nansen.ipsl.jussieu.fr/ AEROCOM/data.html) as supplementary material. Readers are referred to this material to obtain further information on the spatial distributions and their role in explaining the differences in the aerosol life cycles.

\subsection{Concept of model diversity}

A major objective of this paper is to quantify the diversity of the results for the ensemble of global aerosol models taking part in AeroCom. Please note that we employ the term "diversity" to describe the scatter of model results rather than using the term "uncertainty", which indicates a degree of knowledge. The assessment of the uncertainty would, however, necessitate a comprehensive comparison with all kinds of observational data and is out of the scope of this paper. An additional problem is that the participating models are not fully independent from each other, as shown in the tables with the model descriptions, and discussed later in the analysis of the results.

The quantities investigated include aerosol fields and fluxes, atmospheric residence times, water uptake and particle sizes. These are associated with different units and with variations over several orders of magnitude. In order to enable comparability, the data are normalized with the allmodels-average. We express the diversity $\delta$ of the model results in terms of the standard deviation $\sigma$ normalized by the all-models-average in \%:

$\delta=\sigma$ (results/ all-models-average) $\times 100 \quad[\%]$.

The diversities established in this study are valid for the specific AeroCom models considered for their calculations. Data were not always available for all parameters and all models. Please note that the established model diversities can change slightly if new data are added, because the number of models is still quite small for statistical computations. We have also tested other expressions for the model diversity (e.g., median instead of average, percentiles, or the ratio of minimum and maximum results instead of the standard deviation). The absolute values change of course, but the relations among the diversities for different quantities are similar.

Diversities can be compared among different aerosol species for a given quantity, in order to identify weak components that need further attention. However, the diversities of the individual species cannot be simply added to obtain that of total aerosol. For the ideal case of a normally distributed sample of independent quantities, the diversity 

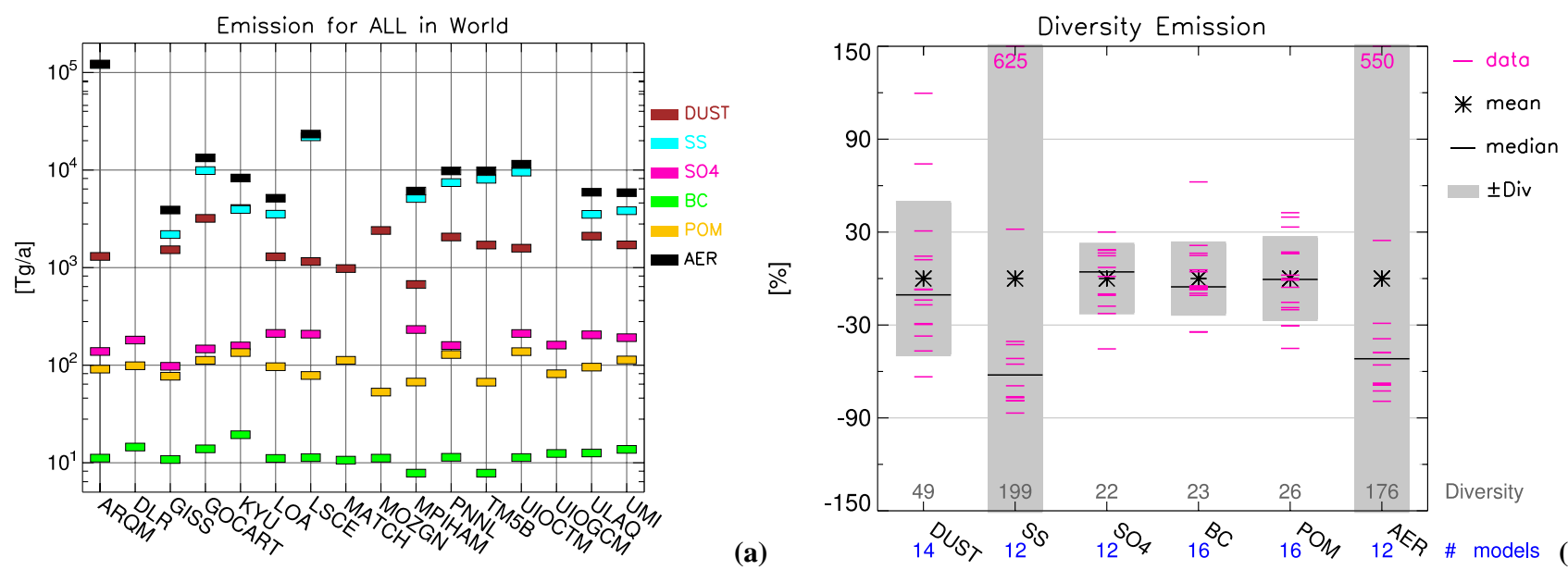

Fig. 1. (a) Global, annual average emissions [Tg/a] in all models for DU, $\mathrm{SS}, \mathrm{SO}_{4}, \mathrm{BC}, \mathrm{POM}$, and $\mathrm{AER}$. For $\mathrm{SO}_{4}$, we show the sum of direct emission and chemical production. (b) Diversity plot for emissions, for further explanations on this plot type see Sect. 3 . The diversity ranges $( \pm \delta)$ are indicated by gray boxes, the numbers for the diversities $(\delta)$ are given in gray in the lower part of the plot for each species. The individual models' deviations from the all-models-averages (see Eq. 3) are plotted as pink lines (data), or as pink numbers if they are outside the scale of the plot. The all-models-averages are indicated by a black star and the medians by a black line. The numbers of models included in the calculation of this statistics are shown in blue below the $\mathrm{x}$-axis. The numbers for the statistics are given in Table 10 .

of total aerosol would be obtained from the square root of the sum of squared standard deviations $\sigma$ of the components, normalized by the all-models-average of AER.

$\delta_{\mathrm{AER}}=\frac{\sqrt{\left(\sigma_{\mathrm{DU}}\right)^{2}+\left(\sigma_{\mathrm{SS}}\right)^{2}+\left(\sigma_{\mathrm{BC}}\right)^{2}+\left(\sigma_{\mathrm{POM}}\right)^{2}+\left(\sigma_{\mathrm{SO}_{4}}\right)^{2}}}{\text { all-models-average }(\mathrm{AER})}$

The diversity of AER is thus always smaller than the sum of the components' diversities. It would be interesting to examine, if the aerosol components are independent, or if there is some tendency among models to simulate more coherent values for AER, so that a compensating effect for the single components can be expected. The comparison of simulated and observed optical depth by Kinne et al. (2006) shows that models perform fairly well for AER in comparison to data, and that the AER results from the different models are quite coherent. However, the model agreement on the contributions from the individual aerosol components, which cannot be constrained by observations, is much less. In this case, the diversity of AER should be smaller than the theoretical value given in Eq. (2). The results from the relatively small ensemble of the AeroCom models are, however, not ideally normally distributed, because similar parameterizations are used, and the number of contributing models is quite small. Therefore, Eq. (2) cannot be strictly applied and we are reluctant to perform such a statistical analysis. Furthermore, the diversities do not provide any information on the relevance of a quantity for the overall aerosol life cycle. This is due to our definition of the diversity as the relative standard deviation using the all-models-average of each parameter for normalization. For example, a high diversity of the simulated dry deposition rates is only significant for the residence time if this process represents an important pathway for the removal. As a consequence, the investigation of diversity propagation is neither straight-forward going from the individual species to total aerosol, nor among different properties of a given species, and diversities have to be handled with care.

In the remainder of the paper, we display the diversities in a homogeneous plot type, where we show a specific diagnostic quantity for all aerosol species studied here, see, e.g., Fig. 1b. In this figure type, the individual models are distinguished, but are plotted as relative deviations of the individual model results from the all-models-average in \%:

$$
\text { data }=\frac{\text { result }- \text { all-models-average }}{\text { all-models-average }} \times 100 \quad[\%] .
$$

The all-models-averages appear at an ordinate value of zero, and the diversity is visualized as the vertical distance $( \pm \delta)$ around them. This presentation facilitates the comparison of the diversities for the different species and processes. The numbers for the statistics of the examined quantities are summarized in Table 10. In addition, we illustrate the results in the form of $x-y$ plots, see, e.g., Fig. 1a, where individual models can be identified. For some models, we are not able to show all diagnostics and missing data appear as gaps in the plots. In DLR, the coarse fraction of SS and DU is neglected. SS fluxes are missing for MATCH, UIO_GCM does not provide fluxes for SS and DU, and the removal fluxes are missing for MOZGN. KYU provided some quantities only for the sum of BC and POM. Size resolved data for the burden, and aerosol water mass is not available for several models. For models where one of the species was not available, we exclude AER from the plots and calculations of 
the diversities. The numbers of the quantities related to the budget are provided on the AeroCom web site. This enables the analysis of specific models and provides a basis for future studies.

\section{Sources}

The aerosol source strength is believed to be one of the major factors causing the differences in the simulated aerosol fields. The sources of the five aerosol species under consideration for all models are shown in Fig. 1a, and the corresponding diversities in Fig. 1b. The total all-models-average aerosol source amounts to $18800 \mathrm{Tg} / \mathrm{a}(\delta=176 \%)$ with the diversity given in parentheses. Sources are dominated by SS with $16600 \mathrm{Tg} / \mathrm{a}(\delta=199 \%)$, followed by DU $(1840 \mathrm{Tg} / \mathrm{a}, \delta=49 \%)$, $\mathrm{SO}_{4}(179 \mathrm{Tg} / \mathrm{a}, \delta=22 \%)$, POM (96.6 Tg/a, $\left.\delta=26.1 \%\right)$, and finally $\mathrm{BC}(11.9 \mathrm{Tg} / \mathrm{a}, \delta=23 \%)$.

The highest diversity is found for the DU and SS. Their source fluxes are prescribed in some of the models and interactively calculated by most others as a function of wind speed, and of soil properties in the case of DU. The parameterizations of the sources are referenced in Table 3 . We find a fairly good agreement on the spatial distribution of SS source fluxes, because emission occurs only over the sea. A model with strong SS emissions in one region tends to have high emissions in all regions. This can be explained by similar distributions of the wind speeds or their gradients in the models. The larger disagreement of the spatial distributions of the DU sources can thus be mainly attributed to the differences in the soil-properties among the models. DU source fluxes have recently been found to be highly sensitive to the high tail of the wind speed distributions (Timmreck and Schulz, 2004). This explains why the source strengths differ also in models that use the same parameterization (e.g., PNNL, ULAQ, and UMI for dust, and LSCE and MPI_HAM for the sea-air transfer for SS). The high sensitivity to the conditions in the environment also becomes evident when comparing the DU source fluxes of LSCE and LOA. These models are based on the same global model, nudged to the same ECMWF reanalysis data for the meteorology, and use the same parameterization. In both models, the DU source fluxes are based on ECMWF winds in the higher spatial resolution of these data and then interpolated to the model grid, where they are modified according to the models' soil moisture. The difference in the DU fluxes of LSCE and LOA is caused by the specific interpolations to the model grid, and by slightly different soil moistures resulting from small discrepancies in the nudging constants for the meteorological data leading to different precipitation fields. Furthermore, models disagree on representation of the particle sizes of SS and DU, especially on the choice of the largest particles simulated, see also Table 4 . This can cause large differences in the emitted mass. However, high emissions associated with large particles are predominantly of local importance, because these particles have fairly short residence times in the atmosphere. We attribute the very high diversity of SS emissions mainly to differences in the simulated particle size. This is especially the case in ARQM, where the emitted SS mass is more than one order of magnitude larger than in the other models.

The emissions of the "anthropogenic" species (BC, POM, and $\mathrm{SO}_{4}$, or their precursors) are prescribed using global inventories providing fluxes from different source types, which are referenced in Table 3. We find lower diversities than for the "natural" components (Fig. 1b). This fairly good agreement does not necessarily imply a good knowledge of this process, but could just mean that the emission inventories used are quite coherent. The production of secondary organic aerosol (SOA) from precursor gases is only simulated in MOZGN, where oxidation of alpha-pinene is computed online within the chemical module. Most models include SOA produced from various gaseous precursor substances in the primary POM emissions, but in some models it is completely neglected. A recent overview on organic aerosol is provided by Kanakidou et al. (2005)

The diversity of the simulated $\mathrm{SO}_{4}$ sources is the smallest among the aerosol species considered here (Fig. 1b), although gas and aqueous phase chemistry is involved in addition to the diversity of gaseous precursor emissions. The methods for the sources of sulfur species are referenced in Table 5. We neglect smaller contributions from $\mathrm{H}_{2} \mathrm{~S}$ and other sulfur species in the AeroCom diagnostics, even if the models take them into account. The chemical production of $\mathrm{SO}_{4}$ is treated with different complexity: in some models it is online-coupled to atmospheric chemistry, others prescribe the distribution of oxidizing species $\left(\mathrm{OH}, \mathrm{O}_{3}, \mathrm{H}_{2} \mathrm{O}_{2}\right)$, or some of it (see Table 5). Figure 2 shows the diversity of the sulfur sources. Please note that we only consider the eleven models that provided both chemical production and precursor gas emissions. Data were missing for ARQM, GISS, UMI, MOZGN, KYU. Furthermore, the diversities of the individual processes have to be weighted by their relevance for the total $\mathrm{SO}_{4}$ source in order to be compared, see also Sect. 3.2. Chemical production is the dominant sulfate source; it contributes on average $97 \%$. Direct $\mathrm{SO}_{4}$ emissions are afflicted with a high diversity, and in four models they are completely neglected (DLR, KYU, UMI, and ULAQ). The emitted sulfur precursor gases consist on average of $79 \%$ $\mathrm{SO}_{2}$ and $21 \%$ DMS. $\mathrm{SO}_{2}$ emissions are prescribed based on anthropogenic emission inventories (see Table 3) whereas DMS emissions are calculated online from global oceanic DMS concentrations fields and sea-air transfer coefficients as a function of wind speed using different parameterizations describing the sea-air transfer. This explains the higher diversity of the DMS emissions. The diversity of the sum of the precursor gases is smaller than that of the individual emissions, indicating that stronger emissions of one of the gases are compensated by weaker emissions of the other gas. See Sect. 3.2 for a discussion on compensating effects. $38 \%$ of the precursor gases are deposited to the ground. The diversity 
Table 3. References for the sources employed for the simulations analyzed in this study.

\begin{tabular}{|c|c|c|c|c|c|c|c|c|c|}
\hline Model & BC & POM & SOA & DUST & Sea Salt & direct $\mathrm{SO}_{4}$ & $\mathrm{SO}_{2}$ & DMS & Other S species \\
\hline ARQM & $\begin{array}{l}\text { Cooke et al. } \\
\text { (1999); Lavoue } \\
\text { et al. (2000); } \\
\text { Liousse et al. } \\
\text { (1996) }\end{array}$ & $\begin{array}{l}\text { Cooke et al. } \\
\text { (1999); Lavoue } \\
\text { et al. (2000); } \\
\text { Liousse et al. } \\
\text { (1996) }\end{array}$ & $\begin{array}{l}\text { included in } \\
\text { POM, } \\
\text { terpene from } \\
\text { Liousse et al. } \\
\text { (1996) }\end{array}$ & $\begin{array}{l}\text { interactively } \\
\text { (Marticorena and } \\
\text { Bergametti, } \\
\text { 1995) }\end{array}$ & $\begin{array}{l}\text { interactively } \\
\text { (Gong, 2003) }\end{array}$ & $\begin{array}{l}\text { monthly anthrop } \\
\mathrm{SO}_{4} \text { GIEA level2 }\end{array}$ & $\begin{array}{l}\text { monthly anthrop } \\
\mathrm{SO}_{2} \text { GEIA level2 }\end{array}$ & $\begin{array}{l}\text { ocean data: } \\
\text { Kettle et al. } \\
\text { (1999), } \\
\text { sea-air transfer: } \\
\text { Wanninkhof } \\
(1992)\end{array}$ & $\begin{array}{l}\text { land } \mathrm{H}_{2} \mathrm{~S} \text { : } \\
\text { Benkovitz and } \\
\text { Schwartz (1997) }\end{array}$ \\
\hline DLR & $\begin{array}{l}\text { fossil fuel } \\
\text { combustion + } \\
\text { bioburn: Cooke } \\
\text { and Wilson } \\
\text { (1996) }\end{array}$ & $\begin{array}{l}\text { fossil fuel } \\
\text { combustion + } \\
\text { bioburn: Liousse } \\
\text { et al. (1996) }\end{array}$ & $\begin{array}{l}\text { included in } \\
\text { POM, terpene } \\
\text { from Liousse et } \\
\text { al. (1996) + } \\
\text { natural terpene } \\
\text { from plants } \\
\text { (Guenther et al., } \\
\text { 1995) }\end{array}$ & $\begin{array}{l}\text { precalculated } \\
\text { monthly means, } \\
\text { (Ginoux et al., } \\
\text { 2001) }\end{array}$ & $\begin{array}{l}\text { interactively } \\
\text { (Monahan et al., } \\
\text { 1986) }\end{array}$ & none & $\begin{array}{l}\text { annual } \\
\text { fossil fuel } \\
\text { combustion: } \\
\text { (Benkovitz et al., } \\
\text { 1994), bioburn } \\
\text { (Hao et al., } \\
\text { 1990), non- } \\
\text { eruptive } \\
\text { volcanoes: (Spiro } \\
\text { et al., 1992), up- } \\
\text { scaled to } \\
\text { 6.7TgS/a }\end{array}$ & $\begin{array}{l}\text { ocean data: } \\
\text { Kettle et al. } \\
\text { (1996), air-sea } \\
\text { transfer: Liss and } \\
\text { Merlivat (1986) }\end{array}$ & none \\
\hline GISS & $\begin{array}{l}\text { industrial: Bond } \\
\text { et al. (2004), } \\
\text { bioburn: Cooke } \\
\text { and Wilson } \\
\text { (1996) }\end{array}$ & $\begin{array}{l}\text { industrial: Bond } \\
\text { et al. (2004), } \\
\text { bioburn: Cooke } \\
\text { and Wilson } \\
\text { (1996) }\end{array}$ & $\begin{array}{l}\text { included in } \\
\text { POM, terpene } \\
\text { from Guenther et } \\
\text { al. (1995) } 10 \% \\
\text { emi rate }\end{array}$ & $\begin{array}{l}\text { interactively } \\
\text { (Cakmur et al., } \\
\text { 2006; Miller et } \\
\text { al., 2006) }\end{array}$ & $\begin{array}{l}\text { interactively } \\
\text { (Monahan et al., } \\
\text { 1986) }\end{array}$ & $2.5 \%$ of $\mathrm{SO}_{2}$ & $\begin{array}{l}\text { Dentener et al. } \\
\text { (2006) except for } \\
\text { biomass \& } \\
\text { aircraft: Koch et } \\
\text { al. (1999) }\end{array}$ & $\begin{array}{l}\text { ocean data: } \\
\text { Kettle et al. } \\
\text { (1999) } \\
\text { air-sea transfer: } \\
\text { Nightingale et al. } \\
(2000)\end{array}$ & none \\
\hline GOCART & $\begin{array}{l}\text { Cooke et al. } \\
\text { (1999); Duncan } \\
\text { et al. (2003); Van } \\
\text { der Werf et al. } \\
\text { (2003) }\end{array}$ & $\begin{array}{l}\text { Cooke et al. } \\
\text { (1999); Duncan } \\
\text { et al. (2003); Van } \\
\text { der Werf et al. } \\
\text { (2003) }\end{array}$ & $\begin{array}{l}\text { Guenther et al. } \\
\text { (1995) }\end{array}$ & $\begin{array}{l}\text { interactively } \\
\text { (Ginoux et al., } \\
\text { 2001; Chin et al., } \\
\text { 2004) }\end{array}$ & $\begin{array}{l}\text { interactively } \\
\text { (Monahan et al., } \\
\text { 1986; Gong et } \\
\text { al., 1997, 2003) }\end{array}$ & $\begin{array}{l}3 \% \text { of anthrop } \\
\mathrm{SO}_{2}\end{array}$ & $\begin{array}{l}\text { anthrop: } \\
\text { seasonal, } \\
\text { bioburn: } \\
\text { monthly, } \\
\text { (Nakicenovic et } \\
\text { al., 2000; Streets } \\
\text { et al., 2003), } \\
\text { non-eruptive } \\
\text { volcanoes } \\
\text { (Andres and } \\
\text { Kasgnoc, 1998) }\end{array}$ & $\begin{array}{l}\text { ocean data: } \\
\text { Kettle and } \\
\text { Andreae (2000), } \\
\text { sea-air transfer: } \\
\text { Liss and Merlivat } \\
\text { (1986) }\end{array}$ & MSA from DMS \\
\hline $\begin{array}{l}\text { KYU } \\
\text { (SPRINT) }\end{array}$ & $\begin{array}{l}\text { Nozawa and } \\
\text { Kurokawa (2006) } \\
\text { based on FAO, } \\
\text { GEIA, HYDE }\end{array}$ & $\begin{array}{l}\text { Nozawa and } \\
\text { Kurokawa (2006) } \\
\text { based on FAO, } \\
\text { GEIA, HYDE }\end{array}$ & $\begin{array}{l}\text { included in } \\
\text { POM, } \\
\text { terpene + NVOC } \\
\text { based on GEIA } \\
\text { adjusted to SOA } \\
\text { emi according to } \\
\text { Griffin et al. } \\
\text { (1999) }\end{array}$ & $\begin{array}{l}\text { interactively } \\
\text { (Gillette, 1978; } \\
\text { Takemura et al., } \\
\text { 2000) }\end{array}$ & $\begin{array}{l}\text { interactively } \\
\text { (Erickson et al., } \\
\text { 1986; Takemura } \\
\text { et al., 2000) }\end{array}$ & none & $\begin{array}{l}\text { anthrop: annual } \\
\text { (Nozawa and } \\
\text { Kurokawa, 2006) } \\
\text { based on A.S.L. } \\
\text { Associates, } \\
\text { HYDE, non- } \\
\text { eruptive } \\
\text { volcanoes } \\
\text { (Andres and } \\
\text { Kasgnoc, 1998), } \\
\text { monthly bioburn } \\
\text { (Spiro et al., } \\
\text { 1992) }\end{array}$ & $\begin{array}{l}\text { interactively } \\
\text { (Bates et al., } \\
\text { 1987; Takemura } \\
\text { et al., 2000) }\end{array}$ & none \\
\hline LSCE & $\begin{array}{l}\text { Generoso et al. } \\
\text { (2003) }\end{array}$ & $\begin{array}{l}\text { Generoso et al. } \\
\text { (2003) }\end{array}$ & $\begin{array}{l}\text { Liousse et al. } \\
\text { (1996) }\end{array}$ & $\begin{array}{l}\text { interactively } \\
\text { (Claquin et al., } \\
\text { 1999; Balkanski } \\
\text { et al., 2003), }\end{array}$ & $\begin{array}{l}\text { interactively } \\
\text { fitted to } \\
\text { Monahan et al. } \\
\text { (1986); Smith } \\
\text { and Harrison } \\
\text { (1998); Schulz et } \\
\text { al. (2004) }\end{array}$ & $\begin{array}{l}5 \% \text { of anthrop } \\
\mathrm{SO}_{2}\end{array}$ & $\begin{array}{l}\text { monthly } \\
\text { EDGARv3.2 } \\
\text { (Olivier, 2002) } \\
\text { except for } \\
\text { shipping: Corbett } \\
\text { et al. (1999); } \\
\text { Mueller (1992); } \\
\text { Hao and Liu } \\
\text { (1994) }\end{array}$ & $\begin{array}{l}\text { ocean data: } \\
\text { Kettle and } \\
\text { Andreae (2000), } \\
\text { sea-air transfer: } \\
\text { Nightingale et al. } \\
(2000)\end{array}$ & $\begin{array}{l}\mathrm{H}_{2} \mathrm{~S}: 3 \% \text { of } \\
\text { anthrop fossil } \\
\text { fuel } \mathrm{SO}_{2} \text {, } \\
\text { MSA from DMS }\end{array}$ \\
\hline LOA & $\begin{array}{l}\text { Reddy et al. } \\
(2005)\end{array}$ & $\begin{array}{l}\text { Reddy and } \\
\text { Boucher (2004) }\end{array}$ & $\begin{array}{l}\text { included in } \\
\text { POM, terpene } \\
\text { from Reddy and } \\
\text { Boucher (2004) }\end{array}$ & $\begin{array}{l}\text { interactively } \\
\text { (Claquin et al., } \\
\text { 1999; Balkanski } \\
\text { et al., 2003) }\end{array}$ & $\begin{array}{l}\text { interactively } \\
\text { (Monahan et al., } \\
\text { 1986; Reddy et } \\
\text { al., 2005) }\end{array}$ & $\begin{array}{l}5 \% \text { of anthrop } \\
\mathrm{SO}_{2}\end{array}$ & $\begin{array}{l}\text { monthly } \\
\text { EDGARv3.2 } \\
\text { (Olivier, 2002) } \\
\text { except for } \\
\text { shipping: Corbett } \\
\text { et al. (1999) and } \\
\text { bioburn: Pham et } \\
\text { al. (1995) }\end{array}$ & $\begin{array}{l}\text { ocean data: } \\
\text { Kettle and } \\
\text { Andreae (2000), } \\
\text { sea-air transfer } \\
\text { (Nightingale et } \\
\text { al., 2000) }\end{array}$ & $\begin{array}{l}\mathrm{H}_{2} \mathrm{~S}: 3 \% \text { of } \\
\text { anthrop fossil } \\
\text { fuel } \mathrm{SO}_{2} \text {, no } \\
\text { direct emi } \\
\text { DMSO, MSA }\end{array}$ \\
\hline MATCH & $\begin{array}{l}\text { Liousse et al. } \\
\text { (1996) }\end{array}$ & $\begin{array}{l}\text { Liousse et al. } \\
\text { (1996) }\end{array}$ & $\begin{array}{l}\text { Liousse et al. } \\
\text { (1996) }\end{array}$ & $\begin{array}{l}\text { Ginoux et al. } \\
\text { (2001); Zender et } \\
\text { al. (2003) }\end{array}$ & NA & $\begin{array}{l}\text { Benkovitz et al. } \\
\text { (1996) }\end{array}$ & $\begin{array}{l}\text { Benkovitz et al. } \\
\text { (1996) }\end{array}$ & $\begin{array}{l}\text { Benkovitz et al. } \\
\text { (1996) }\end{array}$ & $\begin{array}{l}\text { Benkovitz et al. } \\
\text { (1996) }\end{array}$ \\
\hline MPI_HAM & $\begin{array}{l}\text { Dentener et al. } \\
\text { (2006) }\end{array}$ & $\begin{array}{l}\text { Dentener et al. } \\
\text { (2006) }\end{array}$ & $\begin{array}{l}\text { included in POM } \\
\text { (Dentener et al., } \\
\text { 2006) }\end{array}$ & $\begin{array}{l}\text { interactively } \\
\text { (Tegen et al., } \\
\text { 2002) }\end{array}$ & $\begin{array}{l}\text { interactively } \\
\text { fitted to } \\
\text { Monahan et al. } \\
\text { (1986); Smith } \\
\text { and Harrison } \\
\text { (1998); Schulz et } \\
\text { al. (2004) }\end{array}$ & $\begin{array}{l}\text { Dentener et al. } \\
\text { (2006) }\end{array}$ & $\begin{array}{l}\text { anthrop } \\
\text { EDGARv3.2 } \\
\text { (Olivier, 2002) as } \\
\text { Dentener et al. } \\
\text { (2006) }\end{array}$ & $\begin{array}{l}\text { ocean data : } \\
\text { Kettle and } \\
\text { Andreae (2000), } \\
\text { sea-air transfer: } \\
\text { Nightingale et al. } \\
(2000)\end{array}$ & none \\
\hline
\end{tabular}


Table 3. Continued.

\begin{tabular}{|c|c|c|c|c|c|c|c|c|c|}
\hline Model & $\mathrm{BC}$ & POM & SOA & DUST & Sea Salt & direct $\mathrm{SO}_{4}$ & $\mathrm{SO}_{2}$ & DMS & Other S species \\
\hline MOZGN & $\begin{array}{l}\text { fossil fuel: } \\
\text { Cooke et al. } \\
\text { (1999), bioburn: } \\
\text { Mueller (1992); } \\
\text { Hao and Liu } \\
\text { (1994) emi ratios } \\
\text { from Andreae } \\
\text { and Merlet } \\
\text { (2001), biofuel: } \\
\text { EDGARv2.0, } \\
\text { Olivier et al. } \\
\text { (1996) }\end{array}$ & $\begin{array}{l}\text { fossil fuel: } \\
\text { Cooke et al. } \\
\text { (1999), bioburn: } \\
\text { Mueller (1992); } \\
\text { Hao and Liu } \\
\text { (1994) emi ratios } \\
\text { (Andreae and } \\
\text { Merlet, 2001), } \\
\text { biofuel: } \\
\text { EDGARv2.0, } \\
\text { Olivier et al. } \\
\text { (1996) }\end{array}$ & $\begin{array}{l}\text { from oxidation of } \\
\text { alpha-pinene } \\
\text { with } \mathrm{OH}, \mathrm{O}_{3} \text {, } \\
\mathrm{NO}_{3} \text {, toluene } \\
\text { with } \mathrm{OH} \text {, and } \\
\text { higher alkanes } \\
\text { with } \mathrm{OH} \text { : online } \\
\text { coupled to } \\
\text { chemistry: Tie et } \\
\text { al. (2005) }\end{array}$ & $\begin{array}{l}\text { interactively } \\
\text { (Ginoux et al., } \\
\text { 2001), threshold } \\
\text { velocity for wind } \\
\text { erosion: Ginoux } \\
\text { et al. (2004) }\end{array}$ & $\begin{array}{l}\text { interactively (Tie } \\
\text { et al., 2005) }\end{array}$ & $\begin{array}{l}2 \% \text { of anthrop } \\
\mathrm{SO}_{2}\end{array}$ & $\begin{array}{l}\text { monthly mean } \\
\text { bioburn: Mueller } \\
\text { (1992); Hao and } \\
\text { Liu (1994); Emi } \\
\text { ratios from } \\
\text { Andreae and } \\
\text { Merlet (2001), } \\
\text { biofuel: } \\
\text { EDGARv2.0, } \\
\text { Olivier et al. } \\
\text { (1996) }\end{array}$ & $\begin{array}{l}\text { monthly mean } \\
\text { GEIA (Benkovitz } \\
\text { et al., 1996) }\end{array}$ & none \\
\hline PNNL & $\begin{array}{l}\text { fossil fuel from } \\
\text { Cooke et al. } \\
\text { (1999), bioburn } \\
\text { from Cooke and } \\
\text { Wilson (1996), } \\
\text { boreal/temperate } \\
\text { wildfires from } \\
\text { Lavoue et al. } \\
\text { (2000); Olivier } \\
\text { (2002) }\end{array}$ & $\begin{array}{l}\text { fossil fuel + } \\
\text { bioburn (Chuang } \\
\text { et al., 2002), } \\
\text { boreal/temperate } \\
\text { wildfires } \\
\text { (Lavoue et al., } \\
\text { 2000) }\end{array}$ & $\begin{array}{l}\text { included in } \\
\text { POM, } \\
\text { monoterpene } \\
\text { from Guenther et } \\
\text { al. (1995) } 10 \% \\
\text { emi rate }\end{array}$ & $\begin{array}{l}\text { precalculated } \\
\text { monthly mean } \\
\text { IPCC 1999, } \\
\text { Ginoux et al. } \\
\text { (2001) }\end{array}$ & $\begin{array}{l}\text { interactively } \\
\text { (Gong et,al. } \\
\text { 2002) adjusted at } \\
\text { small sizes }\end{array}$ & $\begin{array}{l}3 \% \text { of anthrop } \\
\mathrm{SO}_{2}\end{array}$ & $\begin{array}{l}\text { anthrop: } \\
\text { monthly } \\
\text { EDGARv3.2 } \\
\text { Olivier (2002) } \\
\text { with hi/lo } \\
\text { vertical factors } \\
\text { from seasonal } \\
\text { GEIA 1985 } \\
\text { Sulfur v1B } \\
\text { inventory, } \\
\text { Benkovitz et al. } \\
\text { (1996)., volcanic: } \\
\text { Barrie et al. } \\
\text { (2001) }\end{array}$ & $\begin{array}{l}\text { ocean data: } \\
\text { Kettle et al. } \\
\text { (1999) } \\
\text { sea-air transfer: } \\
\text { Nightingale et al. } \\
\text { (2000), online } \\
\text { winds }\end{array}$ & MSA from DMS \\
\hline TM5 & $\begin{array}{l}\text { Dentener et al. } \\
\text { (2006) }\end{array}$ & $\begin{array}{l}\text { Dentener et al. } \\
\text { (2006) }\end{array}$ & $\begin{array}{l}\text { included in POM } \\
\text { (Dentener et al., } \\
\text { 2006) }\end{array}$ & $\begin{array}{l}\text { Dentener et al. } \\
\text { (2006) }\end{array}$ & $\begin{array}{l}\text { precalculated } \\
\text { (Dentener et al., } \\
\text { 2006), with } \\
\text { ECMWF sea ice } \\
\text { correction }\end{array}$ & $\begin{array}{l}2.5 \% \text { of anthrop } \\
\mathrm{SO}_{2}\end{array}$ & $\begin{array}{l}\text { EDGARv3.2 } \\
\text { (Olivier, 2002) as } \\
\text { Dentener et al. } \\
\text { (2006) }\end{array}$ & $\begin{array}{l}\text { ocean data: } \\
\text { Kettle et al. } \\
\text { (1999), sea-air } \\
\text { transfer: Liss and } \\
\text { Merlivat (1986) }\end{array}$ & $\begin{array}{l}\text { MSA from DMS } \\
\text { added to } \mathrm{SO}_{4}\end{array}$ \\
\hline UIO_CTM & $\begin{array}{l}\text { fossil fuel: } \\
\text { Cooke et al. } \\
\text { (1999), bioburn: } \\
\text { Cooke and } \\
\text { Wilson (1996) }\end{array}$ & $\begin{array}{l}\text { Liousse et al. } \\
\text { (1996) }\end{array}$ & $\begin{array}{l}\text { included in } \\
\text { POM, terpene } \\
\text { from Liousse et } \\
\text { al. (1996) }\end{array}$ & $\begin{array}{l}\text { interactively } \\
\text { (Grini et al., } \\
\text { 2005) }\end{array}$ & $\begin{array}{l}\text { interactively } \\
\text { (Grini et al., } \\
\text { 2002a) }\end{array}$ & $\begin{array}{l}3 \% \text { of anthrop } \\
\mathrm{SO}_{2}\end{array}$ & $\begin{array}{l}1996 \text { anthrop } \\
\text { (Berglen et al., } \\
\text { 2004), GEIA } \\
\text { 1985 scaled to } \\
\text { fossil fuel use, } \\
\text { Europe: EMEP, } \\
\text { ships: Endresen } \\
\text { (2003), } \\
\text { non-eruptive } \\
\text { volcanoes: Spiro } \\
\text { et al. (1992), up- } \\
\text { scaled to } 8 \text { TgS/a }\end{array}$ & $\begin{array}{l}\text { ocean data: } \\
\text { Kettle and } \\
\text { Andreae (2000), } \\
\text { sea-air transfer: } \\
\text { Nightingale et al. } \\
(2000)\end{array}$ & $\begin{array}{l}\mathrm{H}_{2} \mathrm{~S} \text { : Spiro et al. } \\
\text { (1992) }\end{array}$ \\
\hline UIO_GCM & IPCC-TAR 2000 & IPCC-TAR 2000 & none & $\begin{array}{l}\text { prescribed } \\
\text { (Kirkevåg et al., } \\
\text { 2005) }\end{array}$ & $\begin{array}{l}\text { prescribed } \\
\text { (parameterized } \\
\text { from wind speed) } \\
\text { (Kirkevåg et al., } \\
\text { 2005) }\end{array}$ & $2 \%$ of $\mathrm{SO}_{2}$ & $\begin{array}{l}\text { annual anthrop } \\
\text { emis + non- } \\
\text { eruptive } \\
\text { volcanoes } \\
\text { IPCC-TAR } 2000\end{array}$ & $\begin{array}{l}66 \% \text { of } \\
\text { IPCC-TAR } 2000 \\
\text { (DMS->MSA is } \\
\text { not considered) }\end{array}$ & none \\
\hline ULAQ & IPCC-TAR 2000 & IPCC-TAR 2000 & $\begin{array}{l}\text { included in POM } \\
\text { from terpenes } \\
\text { (Liousse et al., } \\
\text { 1996) }\end{array}$ & $\begin{array}{l}\text { precalculated } \\
\text { monthly means, } \\
\text { IPCC-TAR } 2000\end{array}$ & $\begin{array}{l}\text { precalculated } \\
\text { monthly means, } \\
\text { IPCC-TAR } 2000\end{array}$ & $2.5 \%$ of $\mathrm{SO}_{2}$ & $\begin{array}{l}\text { annual anthrop } \\
\text { non-eruptive } \\
\text { volcanoes } \\
\text { IPCC-TAR } 2000\end{array}$ & $\begin{array}{l}\text { monthly means, } \\
\text { IPCC-TAR } 2000\end{array}$ & $\begin{array}{l}\text { OCS ( } 500 \mathrm{pptv}) \\
\text { MSA from DMS }\end{array}$ \\
\hline UMI & $\begin{array}{l}\text { fossil fuel + } \\
\text { biomass BC: } \\
\text { Penner et al. } \\
\text { (1993); Liousse } \\
\text { et al. (1996) }\end{array}$ & $\begin{array}{l}\text { natural: 9\% of } \\
\text { terpene emi from } \\
\text { Guenther et al. } \\
\text { (1995), fossil } \\
\text { fuel + biomass: } \\
\text { (Penner et al., } \\
\text { 1993; Liousse et } \\
\text { al., 1996) }\end{array}$ & $\begin{array}{l}9 \% \text { of natural } \\
\text { POM }\end{array}$ & $\begin{array}{l}\text { precalculated } \\
6 \mathrm{~h} \\
\text { (Ginoux et al., } \\
2001 \text { ) }\end{array}$ & $\begin{array}{l}\text { precalculated } \\
\text { monthly } \\
\text { (Gong et al., } \\
\text { 1997) }\end{array}$ & none & $\begin{array}{l}\text { monthly fossil } \\
\text { fuel + non- } \\
\text { eruptive } \\
\text { volcanoes IPCC- } \\
\text { TAR } 2000\end{array}$ & $\begin{array}{l}\text { Pre-calculated, } \\
\text { monthly, ocean } \\
\text { data (Kettle et } \\
\text { al., 1999), sea-air } \\
\text { transfer: } \\
\text { Nightingale et al. } \\
\text { (2000) }\end{array}$ & None \\
\hline
\end{tabular}

of this loss process is higher than that of the emissions themselves and of similar size as the diversity of chemical $\mathrm{SO}_{4}$ production. Depositional loss of precursor gases is therefore a major reason for the diversity of the simulated $\mathrm{SO}_{4}$ sources. Chemical production takes place in the gas and in the aqueous phase, where the average contribution of the latter is $73 \%$. The diversity of gas phase chemical production is larger than that in the aqueous phase, but the diversity of total chemical production is smaller than those of the two individ- ual pathways. Hence, strong gas phase production could be compensated by weaker aqueous production and vice versa (see also Sect. 3.2).

\section{Aerosol burdens}

In this section we compare the simulated burdens of the aerosol species in the models. The aerosol burdens and the 
Table 4. Sizes of the emitted particles in $\mu \mathrm{m}$ (mass median diameter of log normal distributions, or diameter of size bin for dry particles).

\begin{tabular}{|c|c|c|c|c|c|}
\hline Model & $\mathrm{BC}$ & POM & DUST & SS & $\mathrm{SO}_{4}$ \\
\hline ARQM & 0.1 & 0.1 & $0.01-40.96$ & $0.01-40.96$ & 0.25 \\
\hline DLR & $\begin{array}{l}\text { fossil fuel combustion: } \\
0.08 \text { ( } 75 \% \text { wt } \%), 0.75(25 \% \mathrm{wt} \%) \text {, } \\
\text { bioburn: } \\
0.02(0.01 \mathrm{wt} \%), 0.37 \text { ( } 99.99 \mathrm{wt} \%)\end{array}$ & $\begin{array}{l}\text { fossil fuel combustion: } \\
0.02(2 \% \text { wt } \%), 0.38(98 \% \mathrm{wt} \%) \text {, } \\
\text { bioburn: } \\
0.02(0.01 \mathrm{wt} \%), 0.37(99.99 \mathrm{wt} \%)\end{array}$ & 0.53 & $\begin{array}{l}0.27(0.2 \mathrm{wt} \%) \\
1.88(99.8 \mathrm{wt} \%)\end{array}$ & none \\
\hline GISS & 0.2 & 0.6 & $0.92,2.94,5.88,11.76$ & $0.8,10.0$ & $\begin{array}{l}0.6 \text {, and internally mixed } \\
\text { with dust }\end{array}$ \\
\hline GOCART & 0.078 & 0.174 & $\begin{array}{l}0.28,0.48,0.90,0.16,2.8,4.8,9.0,16.0, \\
0.28-1.6 \text { treated as one size }(1.46) \text { for trans- } \\
\text { port }\end{array}$ & $\begin{array}{l}0.52, \quad 2.38, \quad 4.86 \\
15.0\end{array}$ & 0.312 \\
\hline $\begin{array}{l}\text { KYU } \\
\text { (SPRINT) }\end{array}$ & 0.236 & 0.20 & $\begin{array}{l}0.26,0.40,0.66,1.04,11.64,2.54,4.04 \\
6.40,10.12,16.04\end{array}$ & $\begin{array}{l}0.36,1.12,33.56 \\
11.24\end{array}$ & 0.139 \\
\hline LSCE & 0.14 & 0.34 & 2.5 & 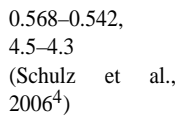 & $\begin{array}{l}0.3 \text { for direct emi and chem- } \\
\text { ical prod }\end{array}$ \\
\hline LOA & 0.1 & 0.3 & $0.02,2,100$ (interval limits) & $\begin{array}{l}0.06,0.012,0.26 \\
0.5,1,2,4,10, \\
20,30,40 \text { (interval } \\
\text { limits at } 80 \% R H \text { ) }\end{array}$ & 0.3 \\
\hline MATCH & 0.1 & 0.14 & tri-modal $0.832,4.82,19.38$ & NA & 0.28 \\
\hline MPI_HAM & $\begin{array}{l}\text { fossil fuel/ biofuel: } 0.069 \\
\text { wildfire: } 0.172\end{array}$ & $\begin{array}{l}\text { fossil fuel/ biofuel: } 0.069 \\
\text { wildfire: } 0.172 \\
\text { biogenic: } 0.069\end{array}$ & coam 3.5, accm 0.74 (Tegen et al., 2002) & $\begin{array}{l}0.568-0.542, \\
4.5-4.3 \\
(\text { Schulz } \quad \text { et } \quad \text { al., } \\
\left.2006^{4}\right)\end{array}$ & $\begin{array}{l}\text { ship, industrial, powerplant: } \\
50 \% \text { accm } 0.175 \text {, } \\
50 \% \text { coam } 1.64 \\
\text { other primary: } \\
50 \% \text { aitm } 0.069 \text {, } \\
50 \% \text { accm } 0.1725\end{array}$ \\
\hline MOZGN & 0.1 & 0.27 & $0.78,2.8,4.8,9,16$ & $0.6,2,6.5,15$ & 0.42 \\
\hline PNNL & $0.19,0.025$ & $0.19,0.025$ & $2.81,0.31$ & $8.45,0.56$ & $0.19,0.025$ \\
\hline TM5 & 0.287 & 0.287 & $1.8,5$ & $0.21,1.3,7.2$ & 0.287 \\
\hline UIO_CTM & $\begin{array}{l}\text { fossil fuel: } 0.10 \\
\text { bioburn: } 0.195,0.852 \\
\text { (except for optics) }\end{array}$ & $\begin{array}{l}\text { fossil fuel: } 0.423 \\
\text { bioburn: } 0.195,0.852 \\
\text { (except for optics) }\end{array}$ & 4.82 & $0.03<\mathrm{d}<25$ & 0.423 \\
\hline UIO_GCM & 0.1 & 0.1 & $0.3,3.78,5.07$ & $0.27,1.88,15.75$ & $\begin{array}{l}0.1 \text { direct emi, homo nucl, } \\
\text { chemical prod added to } \mathrm{DU} \text {, } \\
\mathrm{SS} \mathrm{SO}_{4} \text { in accm }\end{array}$ \\
\hline ULAQ & $\begin{array}{l}0.08,0.02-0.32 \text { ( } 5 \text { bins) normalized size } \\
\text { distribution (Pusechel et al., 1992) }\end{array}$ & $\begin{array}{l}0.28,0.04-0.64 \text { ( } 5 \text { bins) normalized } \\
\text { size distribution as for } \mathrm{SO}_{4}\end{array}$ & $\begin{array}{l}2.56,0.64-10.2 \\
(5 \text { bins })\end{array}$ & $\begin{array}{l}2.56,0.64-20.5(6 \\
\text { bins })\end{array}$ & $\begin{array}{l}0.28,0.0008-20.5 \\
\text { (15 bins) }\end{array}$ \\
\hline UMI & $\begin{array}{l}\text { fossil fuel: } 0.1452 \text { (Radke et al., 1988), } \\
\text { bioburn: } 0.137 \text { (Anderson et al., 1996) }\end{array}$ & $\begin{array}{l}\text { fossil fuel: } 0.1452 \text { (Radke et al., } \\
\text { 1988), } \\
\text { bioburn: } 0.137 \text { (Anderson et al., } \\
\text { 1996) }\end{array}$ & $0.402,1.8,3.488,8.274$ & $\begin{array}{l}0.362, \quad 1.602, \\
3.136,5.916\end{array}$ & $\begin{array}{l}0.0569,0.222,1.529 \\
\text { gas phase prod.: all bins } \\
\text { aqueous prod.: } 2 \text { nd bin }\end{array}$ \\
\hline
\end{tabular}

diversities of the model results are depicted in Figs. $3 \mathrm{a}$ and $\mathrm{b}$, the relevant numbers are given in Table 10.

The AER burden is on average $30.6 \mathrm{Tg}(\delta=29 \%)$ varying from $13.2 \mathrm{Tg}$ to $47 \mathrm{Tg}$. The burdens of DU and SS are $19.2 \mathrm{Tg}(\delta=40 \%)$ and $7.5 \mathrm{Tg}(\delta=54 \%)$, respectively. The AER-burden is dominated by DU followed by SS in all models except for ARQM and MPI_HAM. This is in contrast to the emissions, which are dominated by about one order of magnitude by SS. The diversity of the simulated SS-burdens is much smaller than that of the emissions, mainly because high SS emissions are associated with large SS particles with short residence times, as discussed above. Also for DU, the burden diversity is somewhat smaller than that of the emissions. The burdens of $\mathrm{SO}_{4}$ and $\mathrm{POM}$ are similar with $2 \mathrm{Tg}$ $(\delta=25 \%)$ and $1.7 \mathrm{Tg}(\delta=25 \%)$, respectively, and in 11 of the 16 models the first is more abundant. The burden of BC amounts to $0.24 \mathrm{Tg}(\delta=42 \%)$, and is about one order of magnitude lower than those of $\mathrm{SO}_{4}$ and POM. The ratio of POM to $\mathrm{BC}$ is similar in burdens and emissions. 
Table 5. Sulfur chemistry.

\begin{tabular}{|c|c|c|c|c|}
\hline Model & $\begin{array}{l}\text { Reference for sulfur } \\
\text { cycle }\end{array}$ & Online-coupled to chemistry & Source of prescribed oxidants & Sulfate nucleation \\
\hline ARQM & $\begin{array}{l}\text { von Salzen and } \\
\text { Schlunzen (1999); } \\
\text { Gong et al. (2003) }\end{array}$ & l & $\begin{array}{l}\mathrm{OH}, \mathrm{O}_{3} \text {, and } \mathrm{H}_{2} \mathrm{O}_{2} \text { from MOZART (Brasseur et } \\
\text { al., 1998; Hauglustaine et al., 1998) }\end{array}$ & Kulmala et al. (1998) \\
\hline DLR & Feichter et al. (1996) & l & $\begin{array}{l}\mathrm{OH}, \mathrm{O}_{3}, \mathrm{NO}_{2} \text { (Roelofs and Lelieveld, 1995) } \\
\mathrm{H}_{2} \mathrm{O}_{2} \text {, from Dentener and Crutzen (1993) }\end{array}$ & $\begin{array}{l}\text { homo: Vehkamäki et al. (2002) } \\
\text { hetero: added to accm }\end{array}$ \\
\hline GISS & $\begin{array}{l}\text { Bauer and Koch } \\
(2006) ; \text { Koch et } \\
\text { al. (2006) }\end{array}$ & l & Bell et al. (2005) & $\begin{array}{l}\text { homo: none } \\
\text { hetero: Fuchs and Sutugin (1970) }\end{array}$ \\
\hline GOCART & Chin et al. (2000) & l & $\begin{array}{l}\mathrm{OH}, \mathrm{NO}_{3} \text {, and } \mathrm{H}_{2} \mathrm{O}_{2} \text { from IMAGES (Mueller } \\
\text { and Brasseur, 1995) }\end{array}$ & $\begin{array}{l}\text { homo: none } \\
\text { hetero: added to accm }\end{array}$ \\
\hline $\begin{array}{l}\text { KYU } \\
(\text { SPRINT) }\end{array}$ & $\begin{array}{l}\text { Takemura et al. } \\
(2000)\end{array}$ & I & $\begin{array}{l}\mathrm{OH}, \mathrm{O}_{3} \text {, and } \mathrm{H}_{2} \mathrm{O}_{2} \text { from CHASER (Sudo et al., } \\
\text { 2002) }\end{array}$ & none \\
\hline LSCE & Boucher et al. (2002) & $\begin{array}{l}\text { INCA } \\
\text { (Hauglustaine et al., 2004) }\end{array}$ & I & $\begin{array}{l}\text { homo: none } \\
\text { hetero: added to accm }\end{array}$ \\
\hline LOA & Boucher et al. (2002) & $\mathrm{H} 2 \mathrm{O} 2$ prog & $\begin{array}{l}\text { Pham et al. (1995) with diurnal and monthly vari- } \\
\text { ations }\end{array}$ & $\begin{array}{l}\text { home: none: } \\
\text { hetero: added to accm } \mathrm{SO}_{4}\end{array}$ \\
\hline MATCH & Barth et al. (2000) & I & $\begin{array}{l}\mathrm{OH}, \mathrm{H}_{2} \mathrm{O}_{2} \text {, and } \mathrm{O}_{3} \\
\text { from MOZART (Horowitz et al., 2003) }\end{array}$ & $\begin{array}{l}\text { home:none } \\
\text { hetero:added to accm }\end{array}$ \\
\hline MPI_HAM & Feichter et al. (1996) & l & $\begin{array}{l}\mathrm{OH}, \mathrm{H}_{2} \mathrm{O}_{2}, \mathrm{NO}_{2} \text {, and } \mathrm{O}_{3} \\
\text { from MOZART (Horowitz et al., 2003) }\end{array}$ & $\begin{array}{l}\text { homo: Vehkamäki et al. (2002) } \\
\text { hetero: Fuchs (1959) }\end{array}$ \\
\hline MOZGN & Tie et al. (2005) & $\begin{array}{l}\text { MOZART } \\
\text { (Horowitz et al., 2003) }\end{array}$ & I & $\begin{array}{l}\text { homo: none } \\
\text { hetero: added to accm }\end{array}$ \\
\hline PNNL & $\begin{array}{l}\text { Easter et al. (2004) } \\
\text { except MSA treated } \\
\text { as } \mathrm{SO}_{4} \text {. }\end{array}$ & MIRAGE (Easter et al., 2004) & I & $\begin{array}{l}\text { hetero: Fuchs and Sutugin (1970) } \\
\text { homo: Harrington and Kreidenweis (1998) }\end{array}$ \\
\hline TM5 & $\begin{array}{l}\text { Metzger et al. } \\
(2002 b)\end{array}$ & $\begin{array}{l}\text { TM3 (Houweling et al., 1998; Jeuken } \\
\text { et al., 2001) }\end{array}$ & / & $\begin{array}{l}\text { homo: none } \\
\text { hetero: added to accm }\end{array}$ \\
\hline UIO_CTM & Berglen et al. (2004) & $\begin{array}{l}\text { Berntsen and Isaksen (1997); Berglen } \\
\text { et al. (2004) }\end{array}$ & I & $\begin{array}{l}\text { homo: none } \\
\text { hetero: added to accm bin }\end{array}$ \\
\hline UIO_GCM & $\begin{array}{l}\text { Iversen and Seland } \\
(2002)\end{array}$ & l & Berntsen and Isaksen (1997) & $\begin{array}{l}\text { homo: } 5 \% \text { of gas production } \\
\text { hetero: added internally mixed modes (Iversen } \\
\text { and Seland, 2002; Kirkevåg and Iversen, 2002) }\end{array}$ \\
\hline ULAQ & Feichter et al. (1996) & $\begin{array}{l}\mathrm{OH}, \mathrm{H} 2 \mathrm{O} 2, \mathrm{O} 3, \mathrm{NO} 3 \text { from ULAQ- } \\
\text { CTM (Pitari et al., 2002) }\end{array}$ & / & $\begin{array}{l}\text { homo and hetero } \\
\text { (Pitari et al., 2002) }\end{array}$ \\
\hline UMI & Penner et al. (2002) & $\begin{array}{l}\mathrm{H}_{2} \mathrm{O}_{2} \text { predicted from } \mathrm{HOx} \text { chemistry } \\
\text { and } \mathrm{H} 2 \mathrm{O} 2 \text { photolysis }\end{array}$ & $\begin{array}{l}\mathrm{OH}, \mathrm{HO}_{\mathrm{x}} \text { and } \mathrm{O}_{3} \text { from GRANTOUR } \\
\text { (Penner et al., 1994) }\end{array}$ & $\begin{array}{l}\text { homo: none } \\
\text { hetero: added to the } 2 \text { nd bin }\end{array}$ \\
\hline
\end{tabular}

If the burdens were completely controlled by the emissions we would expect them to have the same diversities. The diversities of the burdens are, however, smaller and can thus only be partly explained by that of the emissions. This indicates that aerosol processes in the atmosphere reduce the effect of diverging sources. This is reflected in the differences of the residence times that are discussed in the next section.

\section{Residence times}

The (tropospheric) residence times reflect the integral of all simulated aerosol properties and processes that affect the burdens, but they are independent of the emissions strengths. Therefore a comparison of the residence times helps to explain the differences in the aerosol fields, which are caused by aerosol processes rather than by contrasting emission strengths. The residence time $\tau$ for an aerosol species is defined as: 


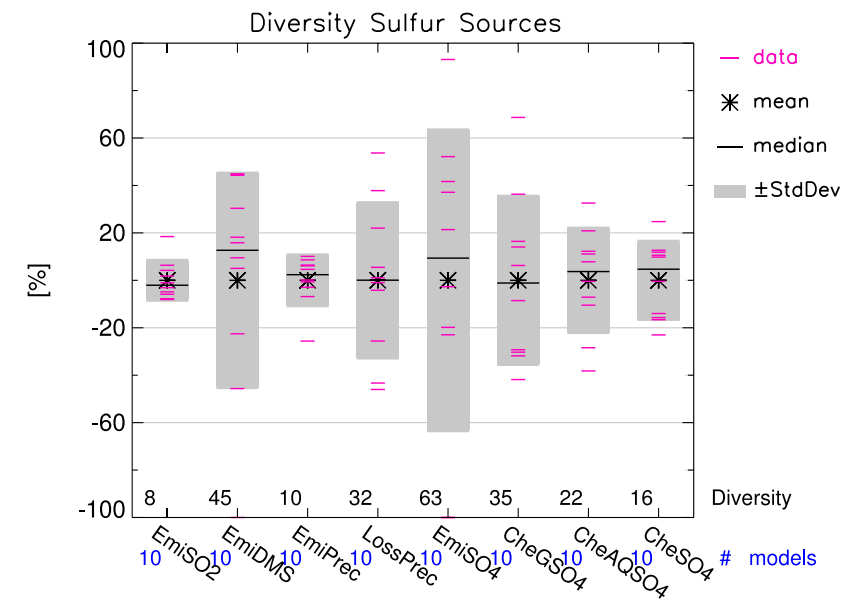

Fig. 2. Diversity plot of the sources of sulfur species. From left to right: $\mathrm{SO}_{2}$-emissions, DMS emissions, sum of precursor $\mathrm{SO}_{2}$ and DMS) emissions, sum of precursor loss by deposition, direct $\mathrm{SO}_{4}$ emissions, chemical production of $\mathrm{SO}_{4}$ in the gas and in the aqueous phase, respectively, total chemical production, and the sum of all sources. For explanations of the plot, please refer to the caption of Fig. 1b.

$\tau=\frac{\text { burden }}{\text { sinks }}$.

The simulated residence times and their diversities are shown in Figs. $4 a$ and b, respectively. The corresponding numbers are given in Table 10.

Sea salt has the shortest $\tau$ of about half a day, followed by $\mathrm{SO}_{4}$ and DU with about four days, and POM and BC with about six and seven days, respectively (see Table 10). The residence times reflect the aerosol properties as they depend on particle size and solubility, but also the model-specific parameterizations of aerosol processes. In addition they reflect the spatial distributions of aerosols in conjunction with those of relative humidity, precipitation, and surface properties. After being emitted, aerosol particles are dispersed by the atmospheric flows of the respective driver models. Transport takes place both through resolved large-scale advection and by parameterized sub-grid scale convective and boundary layer turbulent mixing. Aerosol particles are removed from the atmosphere through wet scavenging, sedimentation and other dry deposition processes. The parameterizations employed to describe the removals in the AeroCom models are briefly discussed in the following section. References can be found in the literature cited in Tables 6 to 8 .

\section{Removal process analysis}

In this section we investigate the individual removal processes and try to understand the differences in residence times both between aerosols of different types and between the various models for a given aerosol type. Specific pro-
Table 6. Sedimentation.

\begin{tabular}{lll}
\hline Model & Components & Reference \\
\hline ARQM & all & Gong et al. (2003) \\
DLR & all & Binkowski and Shankar (1995) \\
GISS & all & Koch et al. (2006) \\
GOCART & all & Fuchs (1964) \\
KYU & all & Takemura et al. (2000) \\
LSCE & all & Schulz et al. (1998) \\
LOA & DU, SS & Reddy et al. (2005) \\
MATCH & DU & Zender et al. (2003) \\
MPI_HAM & all & Stier et al. (2005) \\
MOZGN & DU, SS & Seinfeld and Pandis (1997) \\
PNNL & none & $/$ \\
TM5 & DU, SS & similar to Schulz et al. (1998) \\
UIO_CTM & DU, SS & Grini et al. (2002a, 2005) \\
UIO_GCM & none & $/$ \\
ULAQ & all & Pitari et al. (2002) \\
UMI & all & Seinfeld and Pandis (1997) \\
\hline
\end{tabular}

cesses and parameterizations are identified that cause the simulated aerosol residence times and burdens described above.

In analogy to the differential rate laws of chemical reactions we define the aerosol removal rate as:

$-\frac{d m}{d t}=\tau^{-1} m=k m$,

where $m$ is the aerosol mass, and $t$ the time. The removal rate coefficient $k$ is the inverse of the residence time $\tau$. It is the sum of the individual removal rate coefficients. In the remainder of this paper we distinguish between sedimentation, dry (excluding sedimentation) and wet deposition as shown in Eq. (6):

$k=k_{\mathrm{wet}}+k_{\mathrm{tur}}+k_{\mathrm{sed}}$.

The removal rate coefficients $k_{i}$ for the individual processes can be obtained by multiplication of $k$ with the contributions $f_{i}$ of the individual sink mass fluxes to the total sinks:

$k_{i}=f_{i} \times k$ with $f_{i}=\frac{\text { massflux_sink }_{\mathrm{i}}}{\left.\sum_{i} \text { (massflux_sink } \mathrm{i}_{\mathrm{i}}\right)}$.

The use of these removal rate coefficients isolates differences in the simulated individual removal pathways. In addition, removal rate coefficients are independent from the diversity of the emissions, in contrast to the mass fluxes, which are usually discussed in the context of aerosol life cycles. The single processes compete at each grid point and each time step for available aerosols and are independent from each other (neglecting the additional dependencies introduced from operator splitting here, see Sect. 9.1). However, the removal rate coefficients examined in this paper are obtained from globally and annually averaged mass fluxes 

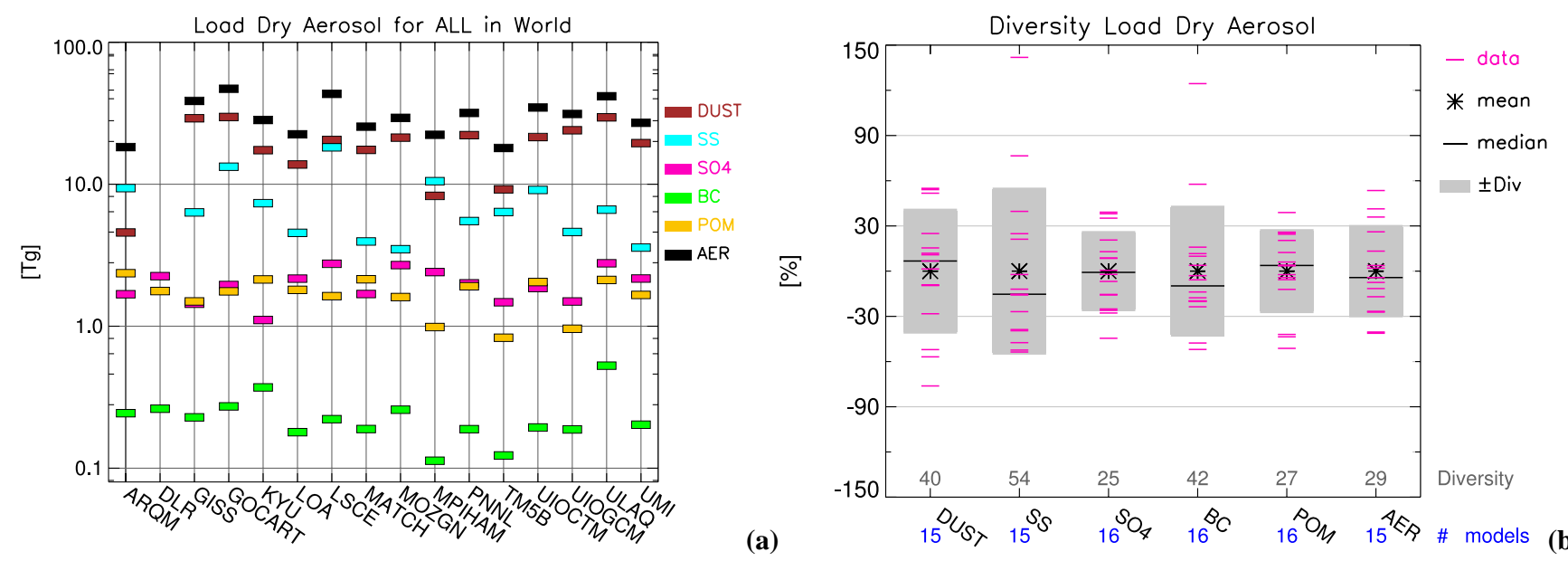

Fig. 3. (a) The global, annual average aerosol burden $[\mathrm{Tg}]$ of the five aerosol species is given for all models. (b) Diversity plot for burdens, for explanations of the plot please refer to the caption of Fig. $1 b$.
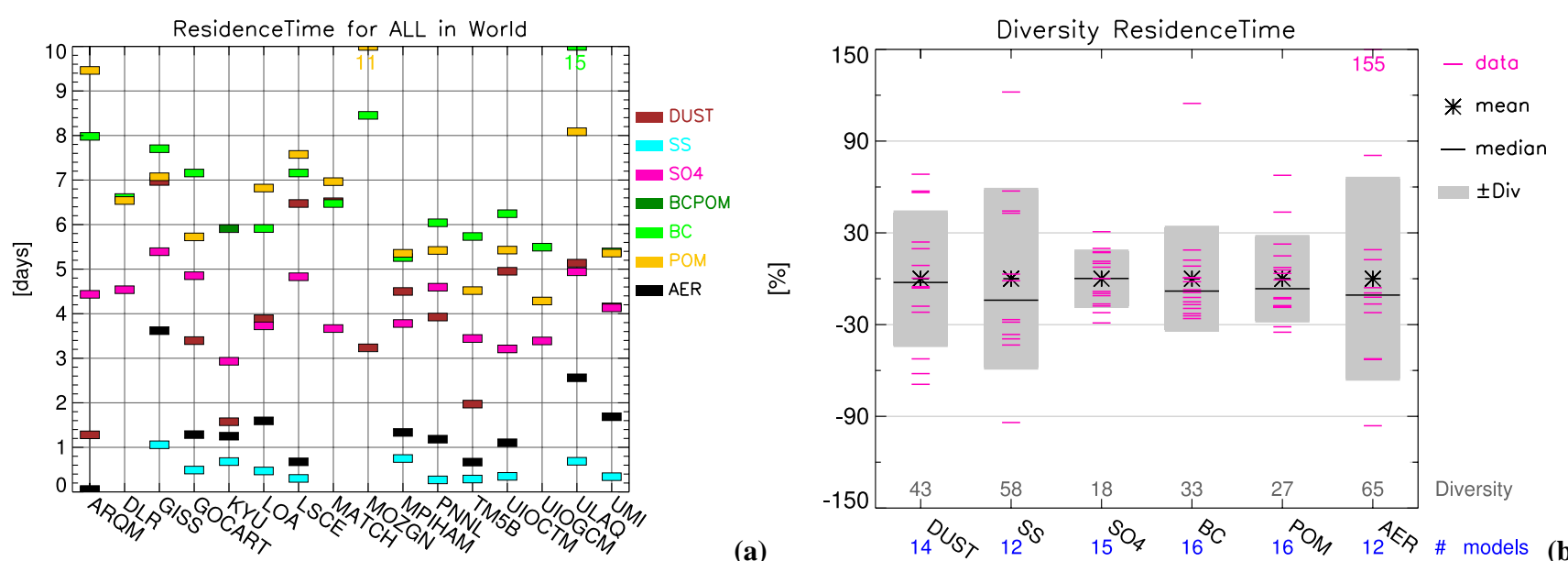

(b)

Fig. 4. (a) Tropospheric residence times in [days] in the AeroCom models for the species under consideration. (b) Diversity plot for residence times, for explanations of the plot please refer to the caption of Fig. $1 b$.

and burdens that are in turn influenced by all other sink processes. Thus, the removal rate coefficients shown in Fig. 5 are not completely independent from each other. However, they average over many applications of the individual parameterizations under all kinds of atmospheric conditions, and thus reflect the overall characteristics of the simulated removal processes.

The wet removal rate coefficients generally increase with the solubility from DU, BC, POM to $\mathrm{SO}_{4}$ and $\mathrm{SS}$. This is reflected in the models by the interstitial fractions of aerosols that are represented with a variety of methods as briefly described in Table 8. Uptake by rain droplets and transfer to cloud droplets depend also on aerosol size, though this effect is simulated in few global models. In addition, the wet removal rate coefficients depend on the degree of concur- rence of the distributions of precipitation and aerosols. Scavenging by ice is considered only in some models. The liquid water or ice concentration used for scavenging is taken from the global model in several cases, sometimes scaled to the cloud-covered fraction of the grid box, or prescribed in others, see Table 8 . The dry deposition (excluding sedimentation) rate is a nonlinear function of particle density and size with a minimum in the accumulation mode. All dry deposition pathways increase with the particle sizes for particles larger than a few tens of microns, but sedimentation becomes increasingly faster than other dry deposition processes for larger particles (diameter larger than a few microns) (Ganzeveld, personal communication, 2005). The rate of other dry deposition processes (i.e., the surface removal due to turbulent transport, Brownian diffusion and impaction, 

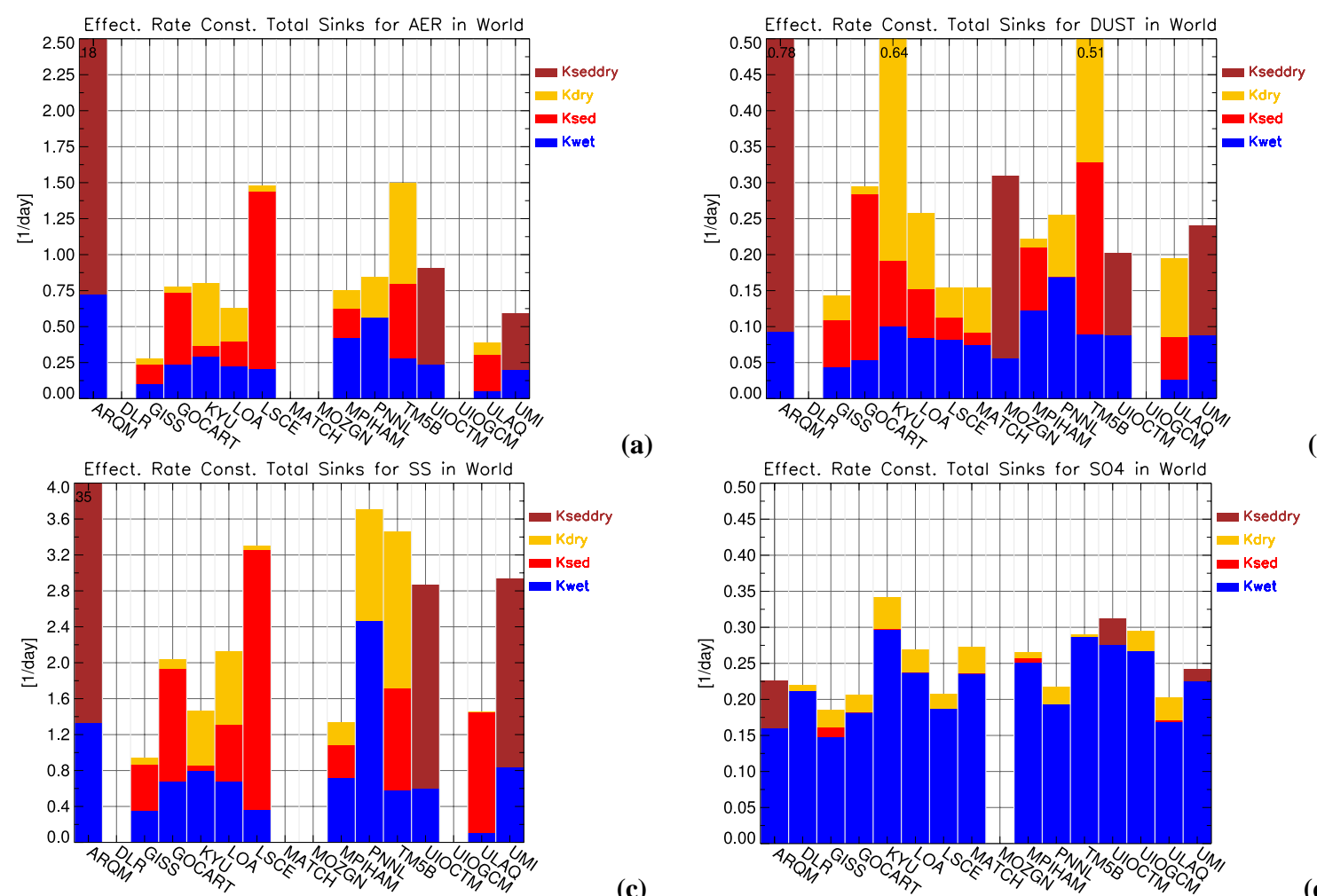

(a)

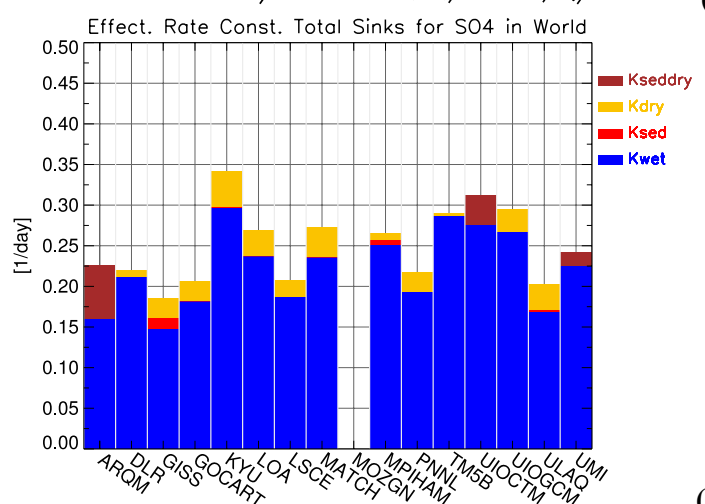

(b)
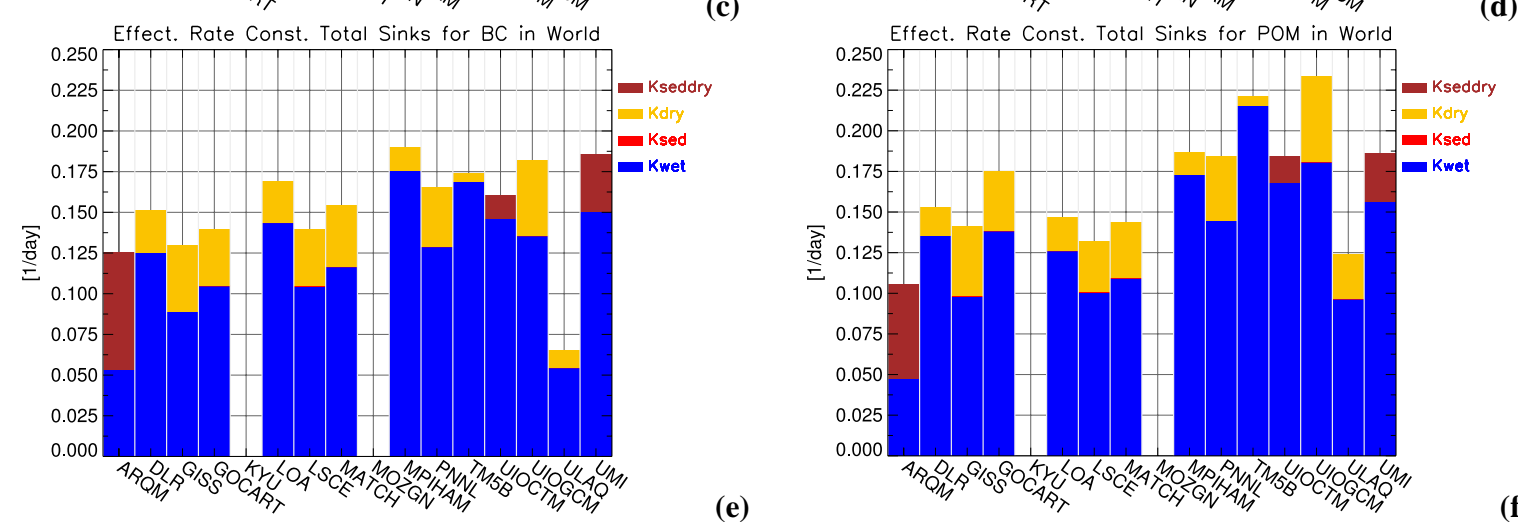

(f)

Fig. 5. Effective sink rate coefficients (annually and globally averaged) for the AeroCom models and for the aerosol species under consideration. The color code is given in the legend: Kwet refers to the wet deposition rate. If possible we show the individual dry sink rate coefficients (Kdry: dry deposition, and Ksed: sedimentation), otherwise the sum of the two processes (KSeddry) is plotted. Please note that the ordinates have different scales.

excluding sedimentation) increases with the aerosol concentration close to the surface and is therefore also a result of the dynamics in the global model. It depends on the intensity of turbulence in the boundary layer, and on the surface properties. In some models, dry deposition is connected to the boundary layer turbulence scheme (e.g., MPI_HAM), others solve it simultaneously with sedimentation (e.g., TM5). Some models use regionally constant dry deposition velocities for all species (e.g., LSCE). In contrast to other dry deposition processes, sedimentation is controlled by the particles properties, as it depends mainly on particle size, density (and shape). It becomes the dominant dry removal process for coarse particles and is therefore ignored in some models for $\mathrm{SO}_{4}, \mathrm{BC}$, and POM. It is neglected for all species in PNNL and UIO_GCM. The particle sizes, and thus the dry deposition rates, are influenced by water uptake in humid ambient air leading to particles growth, for a discussion of this process see Sect. 8.2. The dependence on particle size explains why the all-models-average dry deposition rate of SS is more than ten times larger than that of DU, which is in turn about ten times larger than that of the three "anthropogenic" aerosol types, see Fig. 5. For the removal of "natural" species 
Table 7. Dry deposition (excluding sedimentation).

\begin{tabular}{lll}
\hline Model & Approach & Reference \\
\hline ARQM & resistance & Zhang et al. (2001) \\
DLR & resistance & Binkowski and Shankar (1995); Ganzeveld and Lelieveld (1995) \\
GISS & resistance & Chin et al. (1996); Koch et al. (2006); Schmidt et al. (2005) \\
GOCART & resistance & Wesely (1989), \\
KYU & resistance & Takemura et al. (2000) \\
LSCE & constant velocities & \\
LOA & constant velocities & Boucher et al. (2002); Reddy et al. (2005) \\
MATCH & constant velocities & Rasch et al. (2000) \\
MPI_HAM & resistance & Ganzeveld et al. (1998); Stier et al. (2005) \\
MOZGN & constant velocities & Horowitz et al. (2003); Tie et al. (2005) \\
PNNL & resistance & Zhang et al. (2001) \\
TM5 & resistance & Ganzeveld et al. (1998) \\
UIO_CTM & resistance for DU, SS;constant for BC, POM, SO 4 & Seinfeld and Pandis (1997) \\
UIO_GCM & resistance & Barth et al. (2000) \\
ULAQ & constant velocities & Lohmann et al. (1999) \\
UMI & resistance & Zhang et al. (2001) \\
\hline
\end{tabular}

which are mostly contained in larger particles all three sink processes are important. SS has the highest rate coefficients both for wet and dry deposition, because of its high solubility and large particle size. For the smaller "anthropogenic" particles wet deposition is the dominant sink.

The diversities of the removal rate coefficients simulated for the individual species are shown in Fig. 6. Models agree least for "natural" species, both on the wet and especially on the dry deposition rate coefficients, which can probably be attributed to the contrasting particle sizes. High diversities both for dry and wet deposition are found for the removal rate of AER, transmitted from its components. The results are more coherent for the "anthropogenic" species. As these are predominantly removed by wet deposition, the diversities of the dry deposition rate coefficients are not relevant, and the diversities of their residence times shown in Fig. 4 are mainly due to the scatter in the wet deposition rate.

For the "natural" species, there is no overall agreement among the AeroCom models on whether wet or dry deposition is the dominant removal pathway. Wet deposition contributes on average about one third to the total sinks with fairly high diversities of $\delta=54 \%$ for DU and $\delta=65 \%$ for SS (see Table 10). This diversity of the dominant deposition pathway reflects the disagreement on both wet and dry removal rate coefficients. The diversity of wet deposition rate coefficients $\left(0.08\right.$ days $^{-1}, \delta=42 \%$ for DU and 0.79 days $^{-1}, \delta=77 \%$ for SS) can be attributed to differences in the parameterizations of wet deposition. In some models, DU is internally mixed with other aerosols, thus increasing its solubility. We did not however find systematically enhanced wet deposition of DU in these models. Additional diversity is caused by differences among the models in the simulated distributions of aerosols and precipitation.
The diversities of the total dry deposition rate coefficients are much higher $\left(0.23\right.$ days $^{-1}, \delta=84 \%$ for DU and 4.28 days $^{-1}, \delta=219 \%$ for SS) than for wet deposition. This is probably mainly caused by the large discrepancies of the simulated particle sizes, which then lead to different dry removal rate coefficients (see also the discussion in Sect. 8.1). Furthermore, the models do not agree on the contribution of turbulent deposition and sedimentation for the total dry deposition rate coefficients. Sedimentation contributes on average $46 \%(\delta=66 \%)$ for DU and $59 \%(\delta=65 \%)$ for SS. This finding indicates significant differences in the particle sizes and the parameterizations of these processes. Contrasting contributions of sedimentation are also found for models with large total dry deposition rate coefficients (e.g., DU in KYU and TM5). This might be caused by the parameterizations of the dry deposition processes themselves. Another reason is connected to the aerosol mass at the surface, and thus to the transport provided by global model, and other source and sink processes. The separation of these factors is not within the scope of this survey, and we only consider the sum of the two dry deposition processes in the following.

The high diversity in the contribution of the individual processes to the total removal rate could also be associated with the method of numerically integrating the advectiondiffusion-processes equation. This complex equation is solved numerically in order to obtain the aerosol concentration changes with time. In most current numerical models it is assumed that some or all source and removal (and other) processes are independent from each other. This simplification allows for the separate integration of the single processes in time using a so-called operator splitting method (Marchuk, 1975). Operator splitting is widely used in global numerical models in order to save computational costs. However, the 
Table 8. Wet scavenging.

\begin{tabular}{|c|c|c|c|c|c|c|}
\hline Model & Stratiform clouds & Convective clouds & In-cloud scavenging & Below-cloud scavenging & Scavenging by ice & $\begin{array}{l}\text { Interstitial fraction* of compo- } \\
\text { nents }\end{array}$ \\
\hline ARQM & Lohmann et al. (1999) & Zhang and McFarlane (1995b) & Giorgi and Chameides (1986) & $\begin{array}{l}\text { strat: Slinn (1984) } \\
\text { conv: von Salzen and } \\
\text { Schlunzen (1999) }\end{array}$ & depending on $\mathrm{T}$ & $\begin{array}{l}\text { all: } 0 \\
\text { internally mixed }\end{array}$ \\
\hline DLR & $\begin{array}{l}\text { Lohmann et al. (1999); } \\
\text { Lohmann (2002); } \\
\text { Lohmann and Kärcher } \\
\text { (2002) }\end{array}$ & $\begin{array}{l}\text { Tiedtke (1989) modified after } \\
\text { Brinkop and Sausen } \\
\text { (1997) }\end{array}$ & $\begin{array}{l}\text { strat\&conv: } \\
\text { nucl: impact scav by Brownian mo- } \\
\text { tion, } \\
\text { accm: activation (Henning et al., } \\
\text { 2002), } \\
\text { dust in accm: fix }\end{array}$ & $\begin{array}{l}\text { strat: Berge }(1993) \\
\text { conv: none }\end{array}$ & $\begin{array}{l}\text { strat: } \\
\text { nuclm: none, } \\
\text { accm: } 5 \text { wt } \% \\
\text { conv: none }\end{array}$ & $\begin{array}{l}\text { prog scav coeff } \\
\text { except for DU in accm: } 0.9\end{array}$ \\
\hline GISS & $\begin{array}{l}\text { Del Genio et al. (1996); } \\
\text { Schmidt et al. (2006) }\end{array}$ & $\begin{array}{l}\text { Del Genio and Yao (1993); Del } \\
\text { Genio et al. (2005); Schmidt et } \\
\text { al. (2006) }\end{array}$ & Koch et al. $(1999,2006)$ & Koch et al. (1999) & $\begin{array}{l}\text { strat: } 5 \% \text { liquid } \\
\text { conv: as liquid }\end{array}$ & $\begin{array}{l}\text { strat: } \\
\text { fresh industrial: } \\
\text { BC: } 1, \text { POM: } 11 \text {, } \\
\text { biomass: } \\
\text { POM: } 0.2, \mathrm{BC} 0.4 \text {. } \\
\text { SS: } 0, \mathrm{SO}_{4}: 0 \text {. } \\
\text { conv: } 1 / 2 \text { way between solubil- } \\
\text { ity and } 1 \text {. }\end{array}$ \\
\hline GOCART & $\begin{array}{l}\text { offline } \\
\text { GEOS-DAS } \\
\text { version } 3\end{array}$ & $\begin{array}{l}\text { offline } \\
\text { GEOS-DAS } \\
\text { version } 3\end{array}$ & $\begin{array}{l}\text { Balkanski et al. (1993); Giorgi and } \\
\text { Chameides (1986) }\end{array}$ & $\begin{array}{l}\text { Balkanski et al. (1993); } \\
\text { Giorgi and Chameides } \\
\text { (1986) }\end{array}$ & as liquid & $\begin{array}{l}\mathrm{BC}=0.6, \mathrm{POM}: 0.6, \mathrm{SO}_{4}: 0.6 \text {, } \\
\text { SS: } 0.6 \\
\text { DU: } 0.8\end{array}$ \\
\hline $\begin{array}{l}\text { KYU } \\
\text { (SPRINT) }\end{array}$ & Le Treut and Li (1991) & Arakawa and Schubert (1974) & Takemura et al. (2000) & Takemura et al. (2000) & as liquid & $\begin{array}{l}\text { BC: } 1, \text { OC: } 0.6 \text {, carbonaceous: } \\
0.6, \mathrm{SO}_{4}: 0.5 \text {, SS: } 0.7 \text {, DU: } \\
0.95\end{array}$ \\
\hline LSCE & Le Treut and Li (1991) & $\begin{array}{l}\text { Tiedtke (1989), contributes to } \\
\text { total condensed water }\end{array}$ & Balkanski et al. (1993) & Liu et al. (2001) & as liquid & $\begin{array}{l}\text { BC: } 0.4, \text { POM: } 0.4 \\
\text { SO }_{4}: 0.3, \text { SS: } 0 \\
\text { DU: } 0.5\end{array}$ \\
\hline LOA & Le Treut and Li (1991) & $\begin{array}{l}\text { Tiedtke (1989), contributes to } \\
\text { total condensed water but not to } \\
\text { cloud fraction }\end{array}$ & $\begin{array}{l}\text { Boucher et al. (2002); Reddy et al. } \\
\text { (2005) }\end{array}$ & $\begin{array}{l}\text { Boucher et al. (2002); } \\
\text { Reddy et al. (2005) }\end{array}$ & $\begin{array}{l}\text { as liquid } \\
\text { (in cloud-scav only) }\end{array}$ & all: 0.3 \\
\hline MATCH & Rasch and Kristjansson (1998) & Zhang and McFarlane (1995b) & Rasch et al. (2000) & Rasch et al. (2000) & as liquid & $\begin{array}{l}\text { BC: } 0.8, \text { POM: } 0.8, \mathrm{SO}_{4}: 0.8 \text {; } \\
\text { DU: Zender et al. }(2003)\end{array}$ \\
\hline MPI_HAM & $\begin{array}{l}\text { Lohmann and } \\
\text { Roeckner (1996); } \\
\text { Tompkins (2002) }\end{array}$ & $\begin{array}{l}\text { Tiedtke }(1989) \text { with modifica- } \\
\text { tion for penetrative } \\
\text { convection according to } \\
\text { Nordeng (1994) }\end{array}$ & Stier et al. (2005) & Stier et al. (2005) & Stier et al. (2005) & Stier et al. (2005) \\
\hline MOZGN & Rasch and Kristjansson (1998) & $\begin{array}{l}\text { Hack (1994); Zhang and Mc- } \\
\text { Farlane (1995b) }\end{array}$ & Giorgi and Chameides (1985) & Brasseur et al. (1998) & as liquid & $\begin{array}{l}\text { BC: } 0.8, \mathrm{POM}: 0.8, \mathrm{SO}_{4}: 0.8 \text {, } \\
\text { SS: } 0.8 \mathrm{HNO}_{3}: 1 \text {; DU: Zender } \\
\text { et al. }(2003)\end{array}$ \\
\hline PNNL & $\begin{array}{l}\text { Rasch and Kristjansson (1998); } \\
\text { Zhang (2003) }\end{array}$ & $\begin{array}{l}\text { Hack (1994); Zhang and Mc- } \\
\text { Farlane (1995b) }\end{array}$ & $\begin{array}{l}\text { strat: } \\
\text { activation scav based on mass and } \\
\text { number fraction activated (Abdul- } \\
\text { Razzak, 1998) with Gaussian spec- } \\
\text { trum of updrafts (Ghan et al., 1997), } \\
\text { conv: convective updraft velocity } \\
\text { used }\end{array}$ & $\begin{array}{l}\text { impaction and intercep- } \\
\text { tion (Slinn, 1984) }\end{array}$ & as liquid & $\begin{array}{l}\text { interstitial + activated number } \\
+ \text { mass for each mode pre- } \\
\text { dicted (and transported sepa- } \\
\text { rately) }\end{array}$ \\
\hline TM5 & offline from ECMWF & $\begin{array}{l}\text { offline from ECMWF } \\
\text { (Tiedtke, 1989) }\end{array}$ & $\begin{array}{l}\text { strat: Jeuken et al. (2001) } \\
\text { conv: coupled to updraft mass flux } \\
\text { and conv. precip. }\end{array}$ & $\begin{array}{l}\text { strat: Dana and Hales } \\
\text { (1976) } \\
\text { conv: coupled to updraft } \\
\text { mass flux and conv. pre- } \\
\text { cip. }\end{array}$ & as liquid* 0.2 & $\begin{array}{l}\text { strat: all: } 0.3 \\
\text { conv: all: } 0.0\end{array}$ \\
\hline UIO_CTM & $\begin{array}{l}\text { offline from Inte- } \\
\text { grated Forecast } \\
\text { System (IFS) } \\
\text { ECMWF }\end{array}$ & $\begin{array}{l}\text { mass entrainment/ } \\
\text { detrainment in "elevator" } \\
\text { (Tiedtke, 1989; } \\
\text { Berglen et al., 2004) }\end{array}$ & $\begin{array}{l}\text { soluble: proportional to fraction of } \\
\text { clouds which rains out, } \\
\text { partly soluble: also scaled according } \\
\text { to Henry's law }\end{array}$ & $\begin{array}{l}\text { DU, SS: None } \\
\text { BC, POM, } \mathrm{SO}_{4}: \text { Berge } \\
(1993)\end{array}$ & $\begin{array}{l}\text { as liquid } \\
\text { except for } \mathrm{SO}_{4}\end{array}$ & all: 0 \\
\hline UIO_GCM & Rasch and Kristjansson (1998) & Zhang and McFarlane (1995b) & Iversen and Seland (2002) & $\begin{array}{l}\text { Iversen and Seland } \\
(2002)\end{array}$ & none & $\begin{array}{l}\mathrm{BC}, \mathrm{POM}: 0.5 \\
\mathrm{SO}_{4} \text { : } \\
1 \text { fine particles } \\
0.4 \text { gaseous prod } \\
0.2 \text { aqueous prod strat } \\
0 \text { aqueous prod conv } \\
\text { (Iversen and Seland, 2002) } \\
\text { (DU, SS: prescribed) }\end{array}$ \\
\hline ULAQ & $\begin{array}{l}\text { offline from clima- } \\
\text { tology (Rossow et } \\
\text { al., 1987) }\end{array}$ & $\begin{array}{l}\text { convective mass } \\
\text { fluxes from ECHAM4.L39 } \\
\text { (Grewe et al., 2001) }\end{array}$ & $\begin{array}{l}\text { first order loss as in } \\
\text { Mueller and Brasseur } \\
\text { (1995); BC-OC scav } \\
\text { coeff }=2.1 \mathrm{~cm}-1 \text { (Liousse et al., } \\
\text { 1996); scav coeffs for } \mathrm{SO}_{4} \text {, DU, SS } \\
\text { scaled with factors } 2,0.3,1 \text {, respec- } \\
\text { tively. }\end{array}$ & $\begin{array}{l}\text { first order loss as in } \\
\text { Mueller and Brasseur } \\
\text { (1995); BC-OC scav } \\
\text { coeff }=2.1 \mathrm{~cm}-1 \text { (Liousse } \\
\text { et al., } 1996 \text { ); scav coeffs } \\
\text { for } \mathrm{SO}_{4} \text {, DU, SS scaled } \\
\text { with factors } 2,0.3,1 \text {, } \\
\text { respectively. }\end{array}$ & as liquid & all: 0 \\
\hline UMI & Hack (1998) & $\begin{array}{l}\text { fraction: from Xu } \\
\text { and Krueger (1991), contributes } \\
\text { to total condensed water }\end{array}$ & $\begin{array}{l}\text { Giorgi and Chameides (1986) conv: } \\
\text { convective updrafts as in Rasch et al. } \\
\text { (1997) }\end{array}$ & Balkanski et al. (1993) & as liquid & $\begin{array}{l}\text { BC: } 0.6, \text { POM: } 0.6, \mathrm{SO}_{4}: 0 \text {, } \\
\text { SS: } 0, \text { DU: } 0\end{array}$ \\
\hline
\end{tabular}

*) The interstitial fraction indicates the mass fraction of aerosol which is not scavenged. Insoluble BC and POM (before aging) have interstitial fractions of 1 , except for ULAQ, where they are 0.8 . 

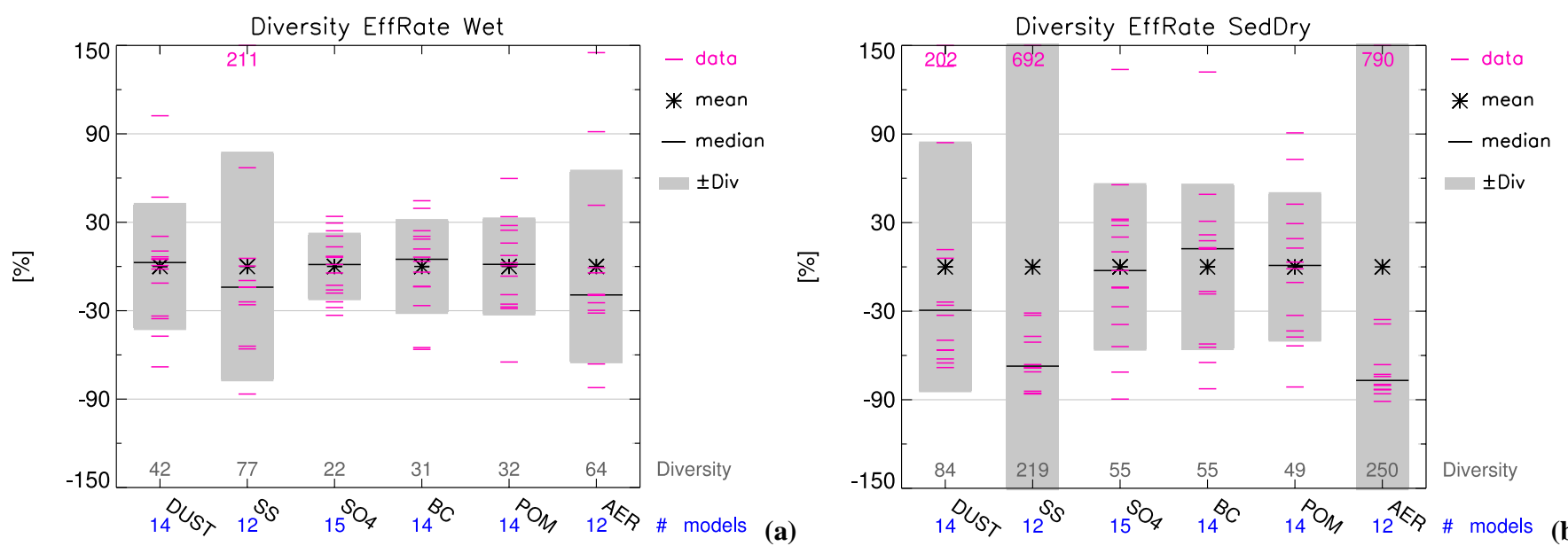

Fig. 6. Diversities plots of the globally and annually averaged effective sink rates coefficients for wet deposition (a) and dry deposition (b). For explanations please refer to the caption of Fig. $1 \mathrm{~b}$.
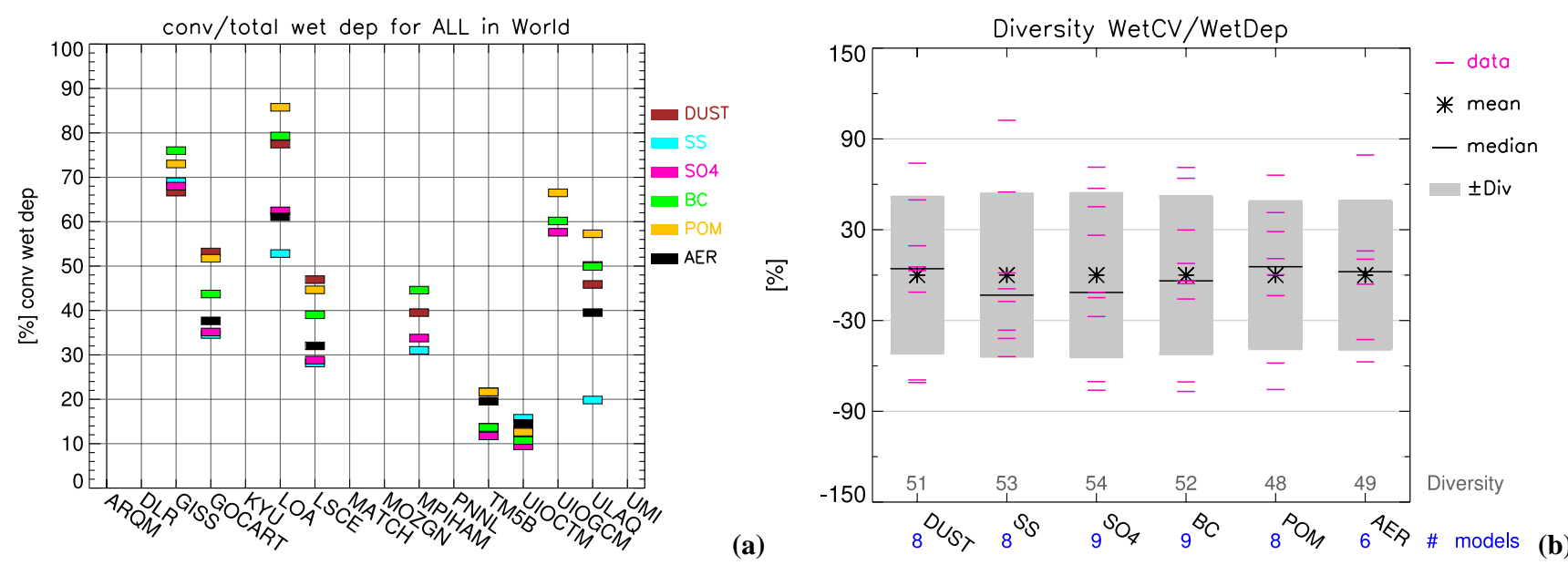

Fig. 7. (a) Mass fractions of global annual convective in relation to total wet deposition (convective + stratiform). (b) Diversity plot for mass fractions of global annual convective in relation to total wet deposition. For explanations please refer to the caption of Fig. $1 b$.

results can be influenced by the sequence of calculating the removal processes thus artificially increasing the contribution of those which are calculated first.

The residence times discussed in Sect. 6 are a result of the different intensities and interactions of the processes involved. It is illustrative to associate these residence times with the individual removal rate coefficients for the single models examined in this section. In ARQM, dry deposition is always faster than in the other models, and it is the dominant sink for all species (except for $\mathrm{SO}_{4}$ ). The short residence times of DU in ARQM, KYU, and TM5 are caused by highly efficient dry deposition while wet deposition is of similar efficiency as in the other models. The models with long DU residence times (GISS, LSCE, and MATCH) have small dry deposition rate coefficients. In GISS, wet deposition is also quite slow, so that this model has the longest life time for DU. PNNL displays the fastest wet deposition rate, but dry deposition is rather slow. (As mentioned above, sedimentation is neglected in PNNL). Therefore, its overall removal rate is in the range of the other models. Dry deposition is the dominant sink for DU in most models apart from LSCE, MPI_HAM, PNNL, and ULAQ. The fast SS-removals in LSCE and in TM5 are caused by relatively large dry deposition rate coefficients. In PNNL, wet deposition is the dominant removal process, it is about three times faster than the all-models-average, and the SS residence time is at the lower end. Wet deposition is also dominant in KYU, MPI_HAM, and ULAQ. However, the wet deposition rate coefficients in these models are well within the range of the other models. Their dry deposition rate coefficients are even slower, and so the SS residence times are rather long. 
The "anthropogenic" aerosols are predominantly removed by wet deposition in all models with the exception of $\mathrm{BC}$ and POM in ARQM. In this model wet deposition is slowest and dry deposition fastest, thus total removal rate of $\mathrm{BC}$ and POM is similar to that in the other models. The contributions of wet deposition to total removal are $79 \%$ $(\delta=17 \%), 80 \%(\delta=16 \%)$, and $89 \%(\delta=8 \%)$ for BC, POM, and $\mathrm{SO}_{4}$, respectively. The wet deposition rate coefficients are $0.12 \mathrm{day}^{-1}(\delta=31 \%)$ for BC, $0.14 \mathrm{day}^{-1}(\delta=32 \%)$ for POM, and 0.22 day $^{-1}(\delta=22 \%)$ for $\mathrm{SO}_{4}$. It is difficult to differentiate between the effects on the scavenging efficiency from particular parameterizations on the one hand, and from the coincidences of different spatial and temporal distributions of aerosols and precipitation on the other hand. The relationship between the spatial distributions and the removal rate is discussed in Sect. 9.

We expect higher wet deposition rate coefficients for the "anthropogenic" species, which are mainly removed by wet deposition, with increased precipitation rates. This should especially be true if the increase is due to a higher rain frequency and not due to heavier single events. The globally and annually averaged precipitation rate (liquid and ice) of the AeroCom-models is between 2.5 and $3.5 \mathrm{~mm}$ per day. We do not find clear correlations between the precipitation rates and the contributions of wet deposition to the total removal, nor between the precipitation rates and the wet deposition rate coefficients (not shown). In some models, however, the scavenging rate is not based on the liquid water content provided by the microphysical scheme of the global model. Instead, a constant liquid water content is used as for example proposed in Giorgi and Chameides (1986). Furthermore, the fractions of the model grid cells that are covered by clouds are treated in various ways. Scavenging in multi-phase clouds and by ice particles is still not well known and thus poorly represented in large-scale aerosol models (e.g., Lohmann and Feichter, 2004). For an overview of the references and parameterizations employed within the AeroCom models for clouds microphysics and aerosol scavenging see Table 8 .

Most models distinguish between in-cloud and belowcloud, and between stratiform (large scale) and convective scavenging, due to the somewhat artificial distinction of cloud types in numerical models. In order to determine the relative importance of the latter two wet deposition pathways we plot in Fig. 7a the contributions of convective wet deposition to total wet depositions for all models for which data are available. The diversity of the results is shown in Fig. 7b. We find similar convective wet deposition efficiencies for aerosols of different type within a given model. However, the AeroCom models do not agree on the rain type which is most efficient in removing aerosols from the atmosphere. In addition, models do not agree on the order in species along which the contribution of convective rain increases. For example for SS we find in some models the highest and in others the lowest contributions of convective wet deposition. The fractions of wet deposition through convective rain range be- tween $10 \%$ and $85 \%$. The diversities of the models results are around $\delta=50 \%$ for all species, in spite of their different properties. These findings indicate that more detailed research is required on wet deposition pathways in global models. As a first step, more information on the simulation of precipitation, especially the types of rain, is needed to separate the effects of the wet scavenging parameterizations from those of the cloud microphysics provided by the global model.

In this section we explained the different residence times for the single aerosol components by their properties and the rate of their individual removal processes. The diversity among the models for the simulated residence times of a given species could be attributed to differences in the representations of individual removal processes. However, we cannot identify in which way the specific parameterizations employed in the models caused the established diversities. Sensitivity studies in a given model examining the effects of different particle size distributions and of different dry deposition parameterizations are needed. In the next section, we examine the effect of the microphysical properties and the spatial distributions of aerosols on the simulated removal rate coefficients.

\section{Particle microphysical properties}

The removal rate coefficients depend on aerosol microphysical properties. In the first part of this section we investigate the simulated particle sizes, and in the second the uptake of ambient water.

\subsection{Sizes}

The description of the particle size distributions depends on the type of the scheme (bulk, modal, or spectral, see Sect. 2.2 and Table 2) and on the number of prognostic variables. The attribution of aerosol mass to three size ranges (diameters of $\mathrm{d}<1 \mu \mathrm{m}, 1 \mu \mathrm{m}<\mathrm{d}<2.5 \mu \mathrm{m}$, and $2.5 \mu \mathrm{m}<\mathrm{d}$ ) was compiled within the AeroCom exercise. The modelers distributed the mass in different ways on these size ranges, based on their model-representation of aerosol sizes. For spectral schemes, the bins within the intervals were simply summed up. In this case the results can be somewhat misleading for schemes with only a few size classes, if these are situated close to the interval boundaries. For modal schemes some participants have used the mass median diameter for classification of the size, and others have more accurately integrated over the distribution within the interval boundaries. In addition, the specific contributions of the accumulation, Aitken and nucleation modes to the fine fraction are not resolved within the AeroCom diagnostics. Furthermore, the results do not document the differences regarding the largest simulated particle sizes, which is especially important for "natural" aerosol as mentioned in Sect. 4. Despite these reservations the analysis of AeroCom data provides for the first time an overview 

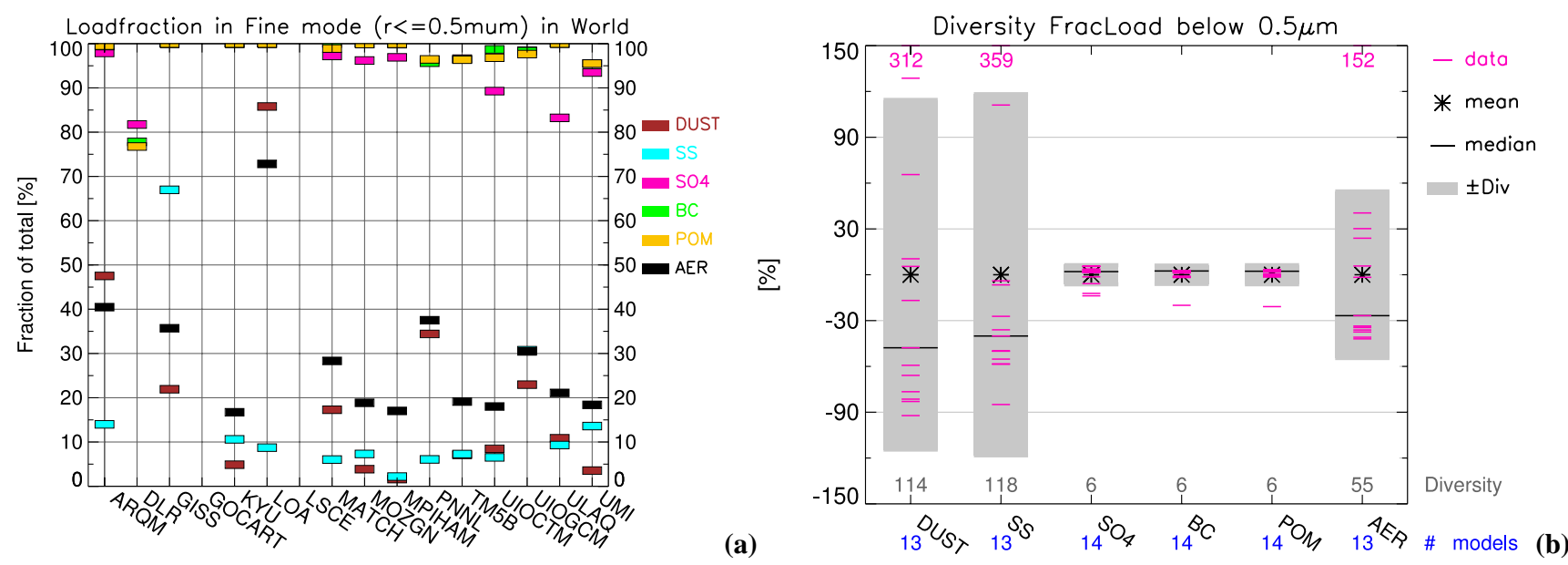

Fig. 8. (a) Mass fraction [\%] in the fine mode (diameter $\mu \mathrm{m}$ ) for DU, SS, $\mathrm{SO}_{4}, \mathrm{BC}, \mathrm{POM}$, and AER. (b) Diversity plot for size fractions, for explanations please refer to the caption of Fig. 1b. (Please note, that there are less models than for most other parameters.)

of the actual size distributions of dry particles and allows for a general view of the diversity of particle sizes in current aerosol modules. For simplification, we focus here on the split of "fine" $(\mathrm{d}<1 \mu \mathrm{m})$ and coarse $(\mathrm{d}>1 \mu \mathrm{m})$ mode particles. In Fig. 8a, we show the mass fractions of particles in the fine mode, and Fig. 8b shows the corresponding model diversities, the numbers are given in Table 10. We concentrate on mass fractions rather than on total burdens in order to remove the effects of contrasting burdens.

The models agree quite well on the mass fractions of "anthropogenic" particles in the fine mode. BC and POM are up to $97 \%$ contained in this size range with very low diversities of $\delta=6 \%$ each. The all-models-average for $\mathrm{SO}_{4}$ in the fine fraction is $95 \%(\delta=6 \%)$. DLR simulates a mass fraction of about $20 \%$ of $\mathrm{SO}_{4}$ larger than the fine mode since the log-normally distributed accumulation mode in this model contains a significant fraction of particles slightly larger than $1 \mu \mathrm{m}$. The fairly large particle size for $\mathrm{SO}_{4}$ found in ULAQ is due to a model artifact. The all-models-average mass fractions for SS, DU and AER in the fine mode are $15 \%$ $(\delta=118 \%), 21 \%(\delta=114 \%)$ and $29 \%(\delta=55 \%)$, respectively. In general, the "natural" components and total aerosol are larger than $1 \mu \mathrm{m}$, but with very high diversities. Models agree better on the fine mass fractions of total aerosol than on those of the "natural" aerosols, by which AER is dominated in mass. This is because in several models a larger fine fraction of one of the "natural" species is compensated by a smaller fine fraction of the other, leading to the smaller diversity of total aerosol (see also Sect. 3.2). Another reason is the additional mass contributing to AER from the more homogeneous size distributions of "anthropogenic" aerosols. The diversity of the fine mass fractions of "natural" aerosols can be associated with specific models: LOA simulates the highest mass fraction of AER in the fine mode ( $>70 \%)$, because of its fine DU. In ARQM, PNNL, and GISS almost $40 \%$ of AER is contained in the fine mode. In the first two this is caused by the small size of DU and in the latter by SS. The differences in the simulated aerosol sizes have important implications for the calculated aerosol radiative forcing, which we do not explore in this paper. The AeroCom data reveal a considerable contribution of "natural" aerosols to the fine fraction, ranging from mass fractions of $10 \%$ in MPI_HAM to $77 \%$ in GISS. Note that this finding indicates that the fine aerosol mode is not purely composed of $\mathrm{SO}_{4}, \mathrm{BC}$, and POM.

We would expect that the simulated SS particle sizes are larger in those models where we find large SS burdens, because these larger particles contribute strongly to the burden but are less relevant for radiative aerosol properties, which are validated against observations. When we compare the mass fractions of SS larger than $2.5 \mu \mathrm{m}$ in diameter with the SS burdens, we do not find a positive correlation. These data do however not resolve the contributions of super-sized SS particles. We conclude that the diversity in the SS burdens is not associated with differences in the simulated particle sizes.

The divergence of the size distributions of "natural" species among the models is partly caused by the sizes of the emitted particles, and partly by the simulated removal processes. We cannot differentiate between these two reasons based on the information available from the AeroCom datasets, because a large fine mass fraction in the burden can either be the reason for slow dry removal rate coefficients for fine particles (calculated here from the burdens and the fluxes), or - in contrast - be the result of a fast dry removal rate for large particles. It would be more suitable to investigate the sizes of emitted particles rather than those of the burdens when examining the relationship between dry removal rate and particle size. More detailed information about the 

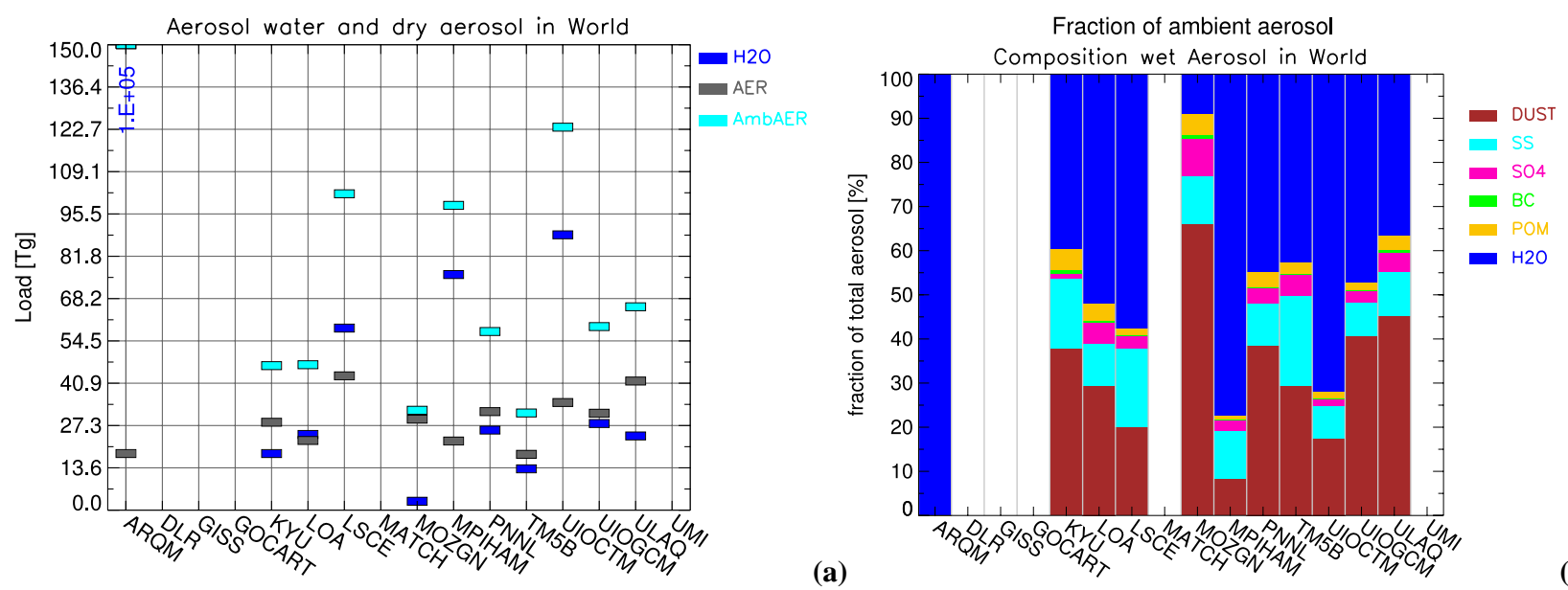

(b)

Fig. 9. (a) Global, annual average masses of dry aerosol $(A E R)$ and of aerosol water $\left(\mathrm{H}_{2} \mathrm{O}\right)$, and ambient aerosol $\left(A m b A E R=A E R+\mathrm{H}_{2} \mathrm{O}\right)$ in [Tg] for all models. (b) Global, annual average composition of ambient aerosol in component mass fractions in relation to total ambient mass $[\%]$.

emitted particle sizes are needed than those summarized in Table 4. In addition, the AeroCom experiment $B$ with unified emissions gives us the opportunity to examine the aerosol processes with lesser effects from contrasting particle sizes.

When we compare the dry deposition rate coefficients with the mass fractions in the fine mode we do not find a clear correlation. (There is also no such correlation for the supercoarse particles fractions with diameters $\mathrm{d}>2.5 \mu \mathrm{m}$.) This can be due to the concerns about the particle size data available for analysis within AeroCom discussed above, but also due to the fact that the investigated sizes refer to dry particles. The simulated removal rate coefficients are actually controlled by ambient particles that grow in the presence of water vapor. Aerosol water uptake is discussed in the next section.

\subsection{Water uptake}

Aerosol particles absorb water depending on their hygroscopicity and the relative humidity in the local environment. Various parameterizations of different complexity are used to describe water uptake by aerosol particles in the ambient atmosphere, for an overview see Table 9. Differences between models are for the first time diagnosed here. The burdens of aerosol water, dry and ambient aerosol are plotted in Fig. 9a. Figure $9 \mathrm{~b}$ shows the composition of ambient aerosol. The corresponding numbers and the diversities of the model results are given in Table 10.

The agreement on the burdens of ambient aerosol (AmbAER) is much less than for the dry aerosol (AER) shown in Fig. 3. The all-models-average aerosol water mass $\left(\mathrm{H}_{2} \mathrm{O}\right)$ (Fig. 9a) is $9480 \mathrm{Tg}$ with a diversity of $\delta=330 \%$, which is mainly caused by the very large $\mathrm{H}_{2} \mathrm{O}$ uptake in ARQM. If we exclude this model, all-models-average $\mathrm{H}_{2} \mathrm{O}$ mass is $35 \mathrm{Tg}$ with a diversity of $\delta=81 \%$. Aerosol water contributes an av- erage mass fraction (Fig. $9 \mathrm{~b})$ of $53 \%(\delta=46 \%)$ to the ambient aerosol composition ranging from $9 \%$ in MOZGN to more than $99.9 \%$ in ARQM. Excluding ARQM we obtain a water fraction of $48 \%(\delta=42 \%)$. (The agreement on the water mass fraction is greater than for the burden, because the high value of ARQM is limited to $100 \%$.)

Water uptake depends on the aerosol composition. Note, that contrasting compositions of the dry aerosol particles are simulated. Therefore, two aerosol models would not obtain the same water uptake, even if they used the same relative humidity and parameterization for hygroscopic growth. Water uptake is most effective for SS containing particles, which is the most hygroscopic aerosol component. Thus, we find an increase of the aerosol water mass (fraction) with increasing $\mathrm{SS}$ mass (fraction) in the model results (not shown). If $\mathrm{H}_{2} \mathrm{O}$ was mainly associated with large, very short-lived SS particles, the high diversity would not be of relevance, e.g., for aerosol radiative forcing. In Sect. 8.1 we show, however, that an all-models-average of $\sim 15 \%$ of the SS burden contains particles in the fine mode leading to significant hygroscopic growth of these particles. More information on how the models attribute $\mathrm{H}_{2} \mathrm{O}$ to the individual aerosol components would be of interest for a follow up study.

Water uptake should increase with increasing local relative humidity for an aerosol of given composition. We compared the simulated annually and globally averaged optical-depthweighted relative humidities and found a range of grid cell averages between $55 \%$ and $77 \%$. There was no relationship between these relative humidities and aerosol water masses for the ensemble of AeroCom models (not shown). However, several models use a local, sub-grid scale relative humidity instead of the grid cell average. The various methods to obtain the local relative humidity represent additional sources for the model diversity (see Table 9). 
Table 9. Hygroscopic growth.

\begin{tabular}{|c|c|c|c|}
\hline Model & Reference & $R H^{*}$ & $R H \max ^{* *}[\%]$ \\
\hline ARQM & Hänel (1976) & mean & 100 \\
\hline DLR & $\begin{array}{l}\text { aerosol liquid water content calculated by EQSAM } \\
\left(\mathrm{SO}_{4} / \mathrm{NH}_{4} / \mathrm{NO}_{3} / \mathrm{H} 2 \mathrm{O} \text { system, (Metzger et al., }\right. \\
2002 \mathrm{a}, \mathrm{b}) \text {, assuming thermodyn equilibrium }\end{array}$ & mean & 99.8 \\
\hline GISS & $\begin{array}{l}\text { Schmidt et al. (2006) based on Tang et al. (1981, } \\
\text { 1996); Tang and Munkelwitz (1991, 1994) }\end{array}$ & mean & $\begin{array}{l}99.9 \\
\text { (not used for radiation) }\end{array}$ \\
\hline GOCART & $\begin{array}{l}\text { d'Almeida (1991) for BC, Koepke et al. (1997) for } \\
\text { the other components }\end{array}$ & mean & none \\
\hline KYU & $\begin{array}{l}\mathrm{SO}_{4} \text { : Tang and Munkelwitz (1994), carbonaceous: } \\
\text { Hobbs et al. (1997), SS: } 30 \% \text { water, DU: hydropho- } \\
\text { bic }\end{array}$ & mean & none \\
\hline LSCE & parameterization adjusted to Gerber (1991) & mean & none \\
\hline LOA & $\begin{array}{l}\mathrm{SO}_{4} \text { Tang and Munkelwitz (1994), SS Tang et } \\
\text { al. (1997), soluble POM as } \mathrm{SO}_{4}\end{array}$ & fraction & 95 \\
\hline MATCH & $\begin{array}{l}\text { POM, } \mathrm{SO}_{4} \text { Hess et al. (1998), Tang and Munkelwitz } \\
\text { (1994) }\end{array}$ & mean & 99.9 \\
\hline MPI_HAM & Vignati et al. (2004) & complex, fraction & complex \\
\hline MOZGN & $\begin{array}{l}\mathrm{SO}_{4} \text { Tang and Munkelwitz (1994), SS Tang et } \\
\text { al. (1997), POM Ming and Russell (2001) }\end{array}$ & mean & 95 \\
\hline PNNL & $\begin{array}{l}\text { Koehler theory, Pruppacher and Klett (1997), Ghan } \\
\text { (2001) for internal mixture of soluble, insoluble } \\
\text { component. Volume mean hygroscopicity. Deliques- } \\
\text { cence depends on } R H \text { and aerosol water from previ- } \\
\text { ous timestep. }\end{array}$ & mean & 100 \\
\hline TM5 & SS: Gerber (1991), $\mathrm{SO}_{4} / \mathrm{NO}_{3}$ Metzger et al. (2002b) & fraction & complex \\
\hline UIO_CTM & Fitzgerald (1975) & mean & 99.5 \\
\hline UIO_GCM & Kirkevåg and Iversen (2002) & mean & 98 \\
\hline ULAQ & as in Kinne et al. (2003) & $\begin{array}{l}\text { daily averaged, } \\
\text { climatological } R H \\
\text { (usually }<90 \% \text { ) }\end{array}$ & 90 \\
\hline UMI & Gerber (1991) for $\mathrm{SO}_{4}, \mathrm{SS}$, and POM & mean & 95 \\
\hline
\end{tabular}

*) Relative humidity used for hygroscopic aerosol growth: grid box mean value (mean) or scaled to cloud-free fraction of grid box (fraction).

**) Relative humidity threshold to distinguish between wet aerosols and cloud droplets.

The rate coefficients of aerosol removal are influenced by hygroscopic growth, because the added water modifies the particle sizes and densities. The diameter of ambient particles with the all-models-average water fraction of about $50 \%$ is about $30 \%$ larger than that of the dry particles, assuming for simplicity the same density for dry aerosol and water. The simulated removal rate coefficients are controlled by these expanded ambient particles. The AeroCom diagnostics do not permit clear association of aerosol water with specific parts of the particle size spectrum in order to investigate the 
Table 10. Statistics of the investigated parameters (The mass of $\mathrm{SO}_{4}$ is given in $\operatorname{Tg}\left(\mathrm{SO}_{4}\right)$ ). The sign "\#” indicates the number of models considered for the calculation of the statistics.

\begin{tabular}{|c|c|c|c|c|c|c|c|c|c|c|c|c|c|}
\hline Parameter & Unit & $\begin{array}{l}\text { DUST } \\
\#\end{array}$ & Mean & Median & $\begin{array}{l}\text { Stdev } \\
{[\%]}\end{array}$ & $\begin{array}{l}\text { SS } \\
\#\end{array}$ & Mean & Median & $\begin{array}{l}\text { Stdev } \\
{[\%]}\end{array}$ & $\begin{array}{l}\text { SO4 } \\
\#\end{array}$ & Mean & Median & $\begin{array}{l}\text { Stdev } \\
{[\%]}\end{array}$ \\
\hline emission & $\mathrm{Tg} / \mathrm{a}$ & 14 & 1840,00 & 1640,00 & 49 & 12 & 16600,00 & 6280,00 & 199 & 12 & 179,00 & 186,00 & 22 \\
\hline burden & $\mathrm{Tg}$ & 15 & 19,20 & 20,50 & 40 & 15 & 7,52 & 6,37 & 54 & 16 & 1,99 & 1,98 & 25 \\
\hline fine mass fraction & $\%$ & 13 & 20,80 & 10,80 & 114 & 13 & 14,60 & 8,72 & 118 & 14 & 94,80 & 96,80 & 6 \\
\hline mass fraction $>5 \mathrm{~km}$ & $\%$ & 15 & 14,10 & 14,10 & 51 & 15 & 8,65 & 6,94 & 92 & 16 & 32.23 & 32.0 & 36 \\
\hline mass fraction in pol, regions & $\%$ & 15 & 1,54 & 1,00 & 102 & 15 & 3,32 & 1,88 & 140 & 16 & 5,91 & 5,60 & 55 \\
\hline residence time & days & 14 & 4,14 & 4,04 & 43 & 12 & 0,48 & 0,41 & 58 & 15 & 4,12 & 4,13 & 18 \\
\hline total removal rate & 1/day & 14 & 0,31 & 0,25 & 62 & 12 & 5,07 & 2,50 & 188 & 15 & 0,25 & 0,24 & 18 \\
\hline wet removal rate & 1/day & 14 & 0,08 & 0,09 & 42 & 12 & 0,79 & 0,68 & 77 & 15 & 0,22 & 0,23 & 22 \\
\hline dry removal rate & 1/day & 14 & 0,23 & 0,16 & 84 & 12 & 4,28 & 1,40 & 219 & 15 & 0,03 & 0,03 & 55 \\
\hline (wet removal)/(total removal) & $\%$ & 14 & 33,00 & 31,70 & 54 & 12 & 30,50 & 30,30 & 65 & 15 & 88,50 & 88,50 & 8 \\
\hline sed/(dry dep) & $\%$ & 10 & 46,20 & 40,90 & 66 & 9 & 58,90 & 59,50 & 65 & 12 & 7,33 & 0,00 & 202 \\
\hline$($ conv,wet dep)/(wet dep) & $\%$ & 8 & 44,50 & 46,40 & 51 & 8 & 34,10 & 29,60 & 53 & 9 & 39,70 & 35,20 & 54 \\
\hline \multirow[t]{2}{*}{ Parameter } & Unit & $\mathrm{BC}$ & & & & POM & & & & AER & & & \\
\hline & & $\#$ & Mean & Median & $\begin{array}{l}\text { Stdev } \\
{[\%]}\end{array}$ & \# & Mean & Median & $\begin{array}{l}\text { Stdev } \\
{[\%]}\end{array}$ & \# & Mean & Median & $\begin{array}{l}\text { Stdev } \\
{[\%]}\end{array}$ \\
\hline emission & $\mathrm{Tg} / \mathrm{a}$ & 16 & 11,90 & 11,30 & 23 & 16 & 96,60 & 96,00 & 26 & 12 & 18800,00 & 9050,00 & 176 \\
\hline burden & $\mathrm{Tg}$ & 16 & 0,24 & 0,21 & 42 & 16 & 1,70 & 1,76 & 27 & 15 & 30,60 & 29,30 & 29 \\
\hline fine mass fraction & $\%$ & 14 & 97,30 & 99,70 & 6 & 14 & 97,00 & 99,20 & 6 & 13 & 28,80 & 21,10 & 55 \\
\hline mass fraction $>5 \mathrm{~km}$ & $\%$ & 16 & 21.20 & 18.30 & 52 & 16 & 20.40 & 18.30 & 56 & 15 & 14,50 & 13,50 & 47 \\
\hline mass fraction in pol, regions & $\%$ & 16 & 4,18 & 4,16 & 71 & 16 & 3,27 & 3,57 & 76 & 15 & 2,45 & 1,98 & 90 \\
\hline residence time & days & 16 & 7,12 & 6,54 & 33 & 16 & 6,54 & 6,16 & 27 & 12 & 1,42 & 1,27 & 65 \\
\hline total removal rate & 1/day & 16 & 0,15 & 0,15 & 21 & 16 & 0,16 & 0,16 & 24 & 12 & 2,27 & 0,79 & 223 \\
\hline wet removal rate & 1/day & 14 & 0,12 & 0,13 & 31 & 14 & 0,14 & 0,14 & 32 & 12 & 0,30 & 0,24 & 64 \\
\hline dry removal rate & 1/day & 14 & 0,03 & 0,04 & 55 & 14 & 0,03 & 0,03 & 49 & 12 & 1,98 & 0,46 & 250 \\
\hline (wet removal)/(total removal) & $\%$ & 14 & 78,60 & 79,50 & 17 & 14 & 79,90 & 78,90 & 16 & 12 & 31,10 & 32,50 & 57 \\
\hline sed/(dry dep) & $\%$ & 11 & 0,47 & 0,00 & 251 & 11 & 0,61 & 0,00 & 198 & 9 & 55,90 & 62,50 & 61 \\
\hline (conv,wet dep)/(wet dep) & $\%$ & 9 & 46,30 & 44,60 & 52 & 8 & 51,60 & 54,50 & 48 & 6 & 34,10 & 34,80 & 49 \\
\hline aerosol water mass & $\mathrm{Tg}$ & & & & & & & & & 11 & 9480,00 & 25,80 & 330 \\
\hline aerosol water mass fraction & $\%$ & & & & & & & & & 11 & 52,50 & 47,20 & 46 \\
\hline
\end{tabular}

relationship between the ambient particle sizes and the removal rate coefficients.

Aerosol water uptake affects the radiative properties of particles as the effective refractive index and the size, both depending on water, determine optical properties of the aerosol. The high diversity in water uptake demonstrated here is thus highly critical for the comparability of the simulation of aerosols climate effects. In addition, the chemical reactivity depends on the available water in the aerosol particle.

\section{Spatial aerosol distributions}

The differences in residence times and dry or wet removal rate coefficients between the models can be better understood by extending the analysis of the burdens to the spatial aerosol distributions. The relatively short residence times of aerosol particles result in large spatial gradients, both vertically and horizontally, in the troposphere. In the remainder of the text we use the term model "dispersivity" to qualitatively characterize the degree of vertical and horizontal aerosol dispersal in a given model, as compared to the other AeroCom models.
As discussed above for the life cycles, the model dispersivity is controlled by the interaction of various removal and transport processes including sub-grid scale mixing. Identical model dispersivities could result from identically simulated transport and internal aerosol processes among models. However, they could also result from different, but mutually compensating transport and aerosol processes. The separation of their effects requires independent information about transport and is therefore beyond the scope of this paper. In the following, we firstly present the zonally average vertical aerosol concentration; next we focus on the vertical dispersivity. Finally we discuss the meridional dispersivity, i.e., long-range transport versus the poles.

\subsection{Zonal mean vertical aerosol concentrations}

In Fig. 10 we show the zonally averaged vertical concentrations of AER for all AeroCom models (except for DLR, in which the coarse fractions of DU and SS are neglected). The differences in the spatial model resolution (see Table 2), ranging from to 304 to 51200 grid points in the horizontal, and from 18 to 40 grid points in the vertical, are evident. 

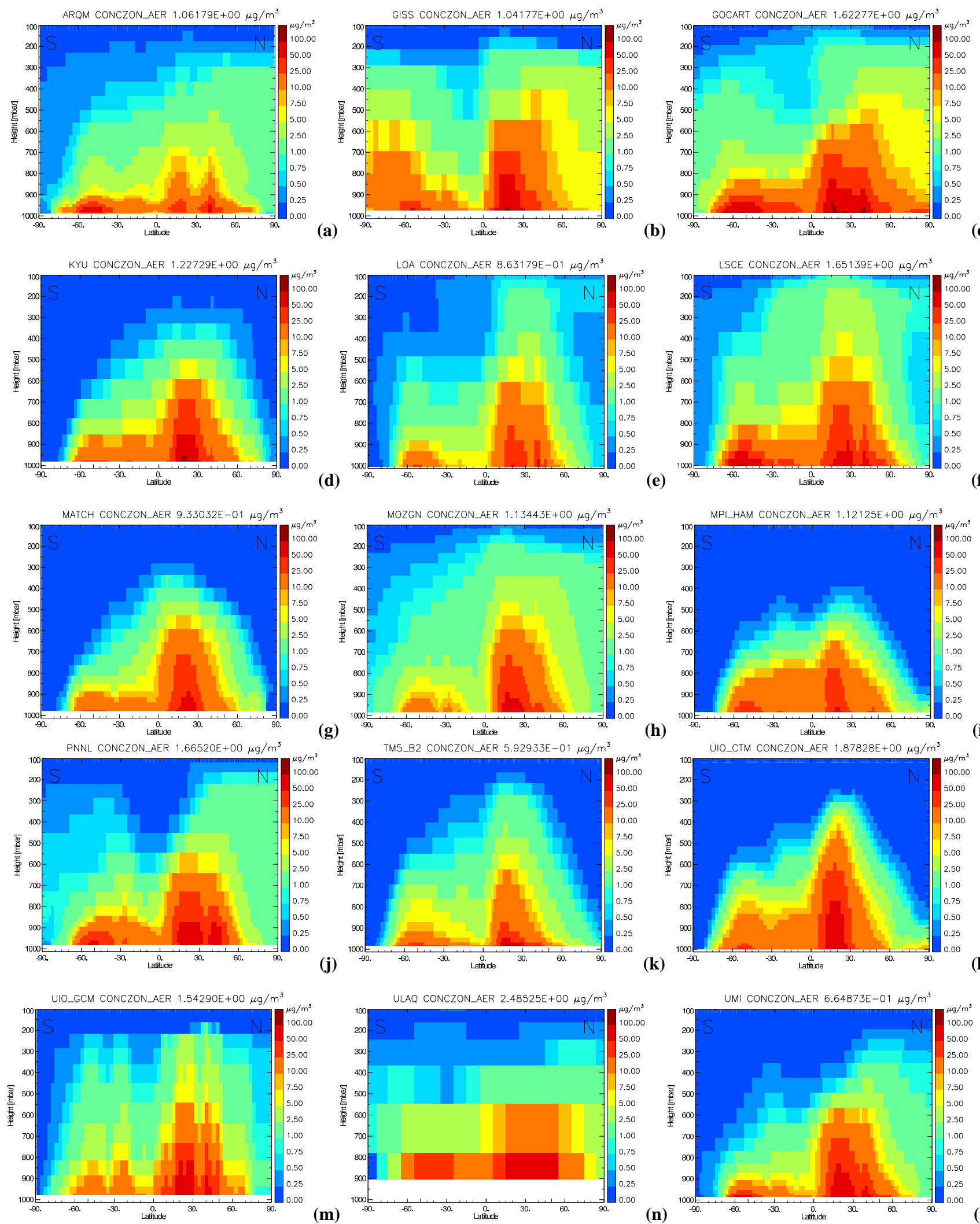

Fig. 10. Zonally and annually averaged concentration of total aerosol in $\left[\mu \mathrm{g} / \mathrm{m}^{3}\right]$ (DLR is not shown because of its disregard of the coarse fractions of SS and DU.) Please note, we use a non-linear color scale. The white shading of lowest layer above ground in some models indicates that no data have been available in this layer. 
The AER concentrations show two maxima in all models: the one in the northern hemisphere results mostly from DU, while the one in the Southern Hemisphere is caused by SS emissions in the "roaring forties" of the South Pacific. However, there are remarkable discrepancies: in some models the aerosol is quite dispersed both in the vertical and in the horizontal direction, in others it is confined to the source regions. The model dispersivity is not linked to the model resolution; compare for example MPI_HAM and MOZGN, or UMI and GOCART that have almost the same resolution. The latter two models also use the same meteorological data, but they still have very contrasting dispersivities, especially regarding the transport towards the poles.

Several models show a minimum of the aerosol concentration in the lowest model layer. This minimum is not easily visible in Fig. 10 for all models concerned, because the lowest layers are sometimes very shallow. Such a minimum is probably not real for the total aerosol concentration, and it is not seen in observational data. Aerosols sometimes show a layered vertical stratification. However, the minima are not located directly at the surface but at higher altitudes (e.g., Bahreini et al., 2003; Kline et al., 2004; Guibert et al., 2005; Meloni et al., 2005). We propose three hypotheses to explain this feature. Firstly, the different parameterizations of dry deposition and their temporal and vertical integrations might play a role in causing this minimum. The dry aerosol removal at the Earth surface also includes the turbulent transport from a reference height in the surface layer $(\sim 10 \%$ of planetary boundary layer (PBL) depth) to the surface where deposition occurs. Overestimation of surface removal together with underestimation of downward turbulent transport in the PBL, which would compensate for the efficient surface removal, could cause a minimum close to the surface. In contrast, there could also be potential too fast upward transport of the emitted species explaining the possible underestimation of surface layer concentrations. The third reason for the surface minimum could be operator splitting, see Sect. 7. We presume that the surface minimum concentration can especially be found in those models in which the diagnostic output is obtained just after calculating the aerosol removal processes. We cannot test our hypotheses with the information available, and the causes for the minimum in surface concentration seen in the AeroCom model results need further attention. This is particularly important, as the simulated aerosol concentrations at the surface are compared to observational data for model validation purposes. Further analysis of a comparison of models to surface observations is provided in Guibert et al. $\left(2006^{1}\right)$.

The AER distributions shown in Fig. 10 are dominated by the masses of DU and SS. In the following we investigate the spatial distributions of the individual species, first focusing on the vertical dispersivity.

\subsection{Vertical dispersivity}

Wet scavenging becomes increasingly less significant when aerosols reach altitudes where the clouds show decreased precipitation efficiency (see also Lohmann et al., 1999). Especially small particles, for which sedimentation is not significant, can in that case have rather extended residence times. We choose for diagnostic purposes a characteristic height of $5 \mathrm{~km}$ and discuss the mass fractions above this height as an indicator for the vertical dispersivity, see Fig. 11. Additional layers of different depth $(0-1,1-2.5,2.5-5 \mathrm{~km})$ are discussed in the text.

The ranges of vertical dispersivity, i.e., the differences for each model between the species with the largest and the smallest mass fractions above $5 \mathrm{~km}$, respectively, are indicated by the gray shadings in Fig. 11a. These ranges, which indicate the degree of similarity of the vertical dispersivity among the species within a given model, differ among the models. The all-models-average range is $25 \%(\delta=37 \%)$, varying from $10 \%$ in KYU to $45 \%$ in PNNL. In general, KYU, MATCH, MPI_HAM, and TM5 show characteristically lower vertical dispersivities for all species than LOA, LSCE, MOZGN, and UIO_GCM. LSCE and LOA are based on the same global model, the French GCM (LMDzT) (see Table 2). Assuming that the effects of differences in precipitation resulting from slightly different nudging constants in these two models are small (see Sect. 4), we can mainly attribute the deviations of their vertical dispersivities to differences in the parameterizations of aerosol processes. The highest diversities of vertical dispersivities among model results are found for SS, followed by POM, BC, DU, and $\mathrm{SO}_{4}$, see Fig. $11 b$.

Vertical dispersivity is weaker for the "natural" species. The all-models-average mass fractions of SS and DU above $5 \mathrm{~km}$ are $9 \%(\delta=92 \%)$, and $14 \%(\delta=51 \%)$, respectively. The SS masses decrease most with height, with largest gradients in MATCH, MPI_HAM, PNNL, and UMI, where more than $90 \%$ of the SS mass are within the planetary boundary layer (PBL) below an altitude of $2.5 \mathrm{~km}$. SS reaches greater heights in LOA, LSCE, UIO_GCM, and especially in MOZGN, where $25 \%$ of SS are above $5 \mathrm{~km} .15 \%(\delta=47 \%)$ of AER occur above $5 \mathrm{~km}$ height. The AER composition close to the surface below $1 \mathrm{~km}$ is dominated by SS in eight models and by DU in seven models. In this layer, the SS contribution to the AER composition varies from about $20 \%$ to $80 \%$, and DU contributes between $15 \%$ and $70 \%$. All models have a DU maximum in the upper PBL from 1 to $2.5 \mathrm{~km}$, where DU is the dominant species in all models (mass fractions from $50 \%$ to $80 \%$ ), except for ARQM and MPI_HAM, where SS is still dominant. Although the aerosol particle ascent in the atmosphere depends on its size, we do not find a correlation between the sizes and the mass fractions of DU or SS above $5 \mathrm{~km}$ for the ensemble of AeroCom models (not shown). It might nevertheless exist, but is probably not evident in information on the size distribution as we have argued in Sect. 8.1. 

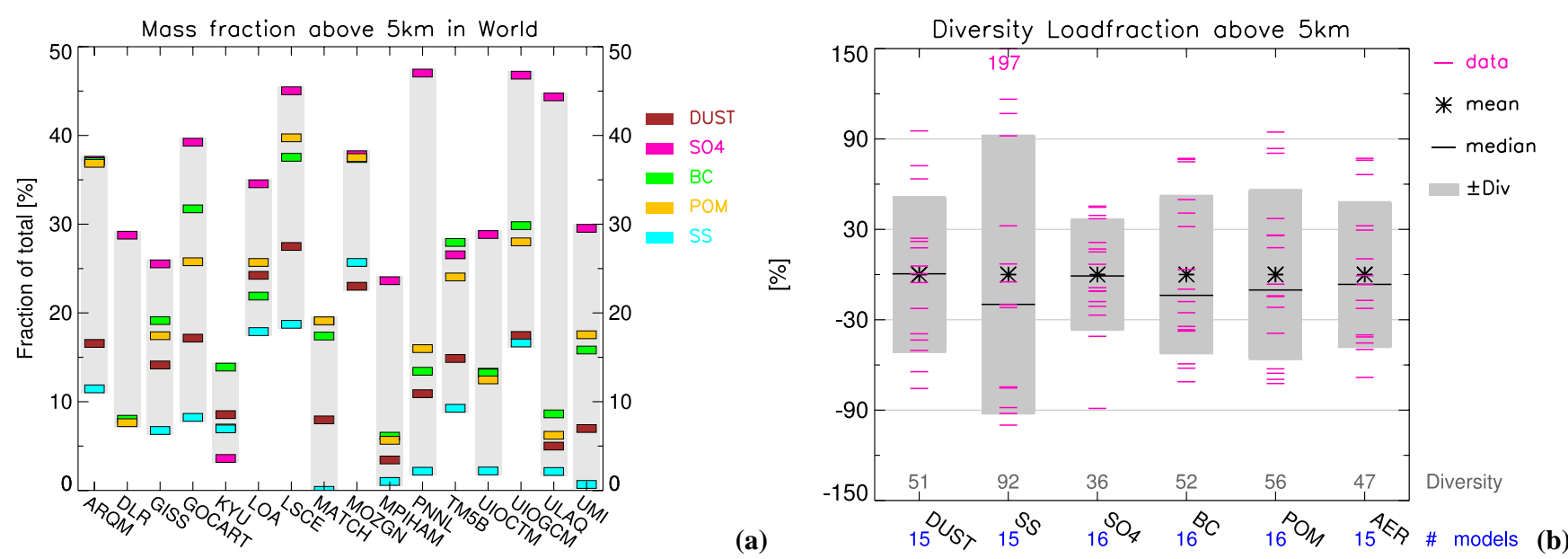

Fig. 11. (a) Global, annual average mass fractions in [\% ] of total mass above $5 \mathrm{~km}$ altitude for the AeroCom models. The ranges of vertical dispersivity, i.e., the differences for each model between the species with the largest and the smallest mass fractions above $5 \mathrm{~km}$, respectively, are indicated by the gray shadings. (b) Diversity plot of the annual average aerosol mass fractions above $5 \mathrm{~km}$ altitude, for explanations please refer to the caption of Fig. 1b.

The vertical distributions of BC and POM are similar in most models; larger differences are found in TM5, GOCART, and LOA. The two species are well dispersed in the vertical with the maximum situated in the upper PBL between 1 and $2.5 \mathrm{~km}$ in all models. The all-models-average mass fractions above $5 \mathrm{~km}$ are $21 \%(\delta=52 \%)$ and $20 \%$ ( $\delta=56 \%$ ) for BC and POM, respectively. In eight models, the vertical dispersivity is stronger for BC than for POM, in six models the situation is reversed, and in two models similar. There are several reasons for the differences of the simulated vertical distributions of BC and POM within a given model. The formation of secondary POM from organic precursor gases within the atmosphere, if included in the model, increases the amount of POM at greater heights. At the same time, the greater solubility of this species leads to an increased wet scavenging rate, thus reducing highaltitude-POM. Finally, divergent spatial distributions of the two species resulting from transport influence their deposition rate coefficients, which in turn affect the spatial distributions. Weaker vertical dispersivity of BC than of POM could explain the faster wet deposition rate coefficients of this species in three models (LOA, LSCE, MATCH). However, not all models with weaker vertical dispersivity for BC show faster wet removal rate coefficients for this species.

$\mathrm{SO}_{4}$ is present at the highest altitudes of all species (exceptions: TM5 and especially KYU). The all-models-average $\mathrm{SO}_{4}$ mass fraction above $5 \mathrm{~km}$ is $32 \%(\delta=36 \%)$. The main $\mathrm{SO}_{4}$ burden is situated in the upper PBL between $1-2.5 \mathrm{~km}$ in nine models, in the lower free troposphere between 2.5 and $5 \mathrm{~km}$ in three models, in the upper free troposphere between 5 and $10 \mathrm{~km}$ in one model, and above $10 \mathrm{~km}$ in the tropopause region in three models. In $\mathrm{KYU}, \mathrm{SO}_{4}$ is the aerosol species which is most confined to lower levels, and only $\sim 5 \%$ can be found above $5 \mathrm{~km}$. In general, the $\mathrm{SO}_{4}$-contribution to the AER composition becomes increasingly important with height due to the removal of DU and SS, and due to chemical $\mathrm{SO}_{4}$-production at greater altitudes within the atmosphere. In several models, $\mathrm{SO}_{4}$ dominates the aerosol composition above $10 \mathrm{~km}$, and injection into the stratosphere is probable. Its vertical distribution depends on the distributions of the emissions, on the chemical production, on the distribution of clouds and precipitation, on the parameterization of the wet deposition process, and on transport provided by the global model. An extensive analysis of sulfur cycle simulations in large scale atmospheric models was performed in the COSAM exercise (Barrie et al., 2001; Lohmann et al., 2001; Roelofs et al., 2001). The authors concluded that the uncertainty in predicting the global $\mathrm{SO}_{4}$ distribution is related to vertical mixing of emitted sulfur species from the planetary boundary layer into the free troposphere. In addition, they suggested that cloud physics and cloud distributions play a major role as they influence cloud-related processes, i.e., the aqueous oxidation of $\mathrm{SO}_{2}$ and wet deposition. Our results confirm the sensitivity of the sulfur cycle to the vertical distribution, which in turn acts on the efficiency of both the aqueous phase production and the removal rate coefficients. In addition, chemical production of $\mathrm{SO}_{4}$ at high altitudes explains why its mass fractions at higher altitudes are higher than those of DU, although these components have similar atmospheric residence times.

We would expect slower removal rate coefficients in models where vertical dispersivity is stronger. We do find such a correlation for the "anthropogenic" aerosols, where wet deposition is the dominant removal, but not for SS and DU, which are by two thirds removed through dry deposition. Dry deposition rate increases with increasing concentrations in 

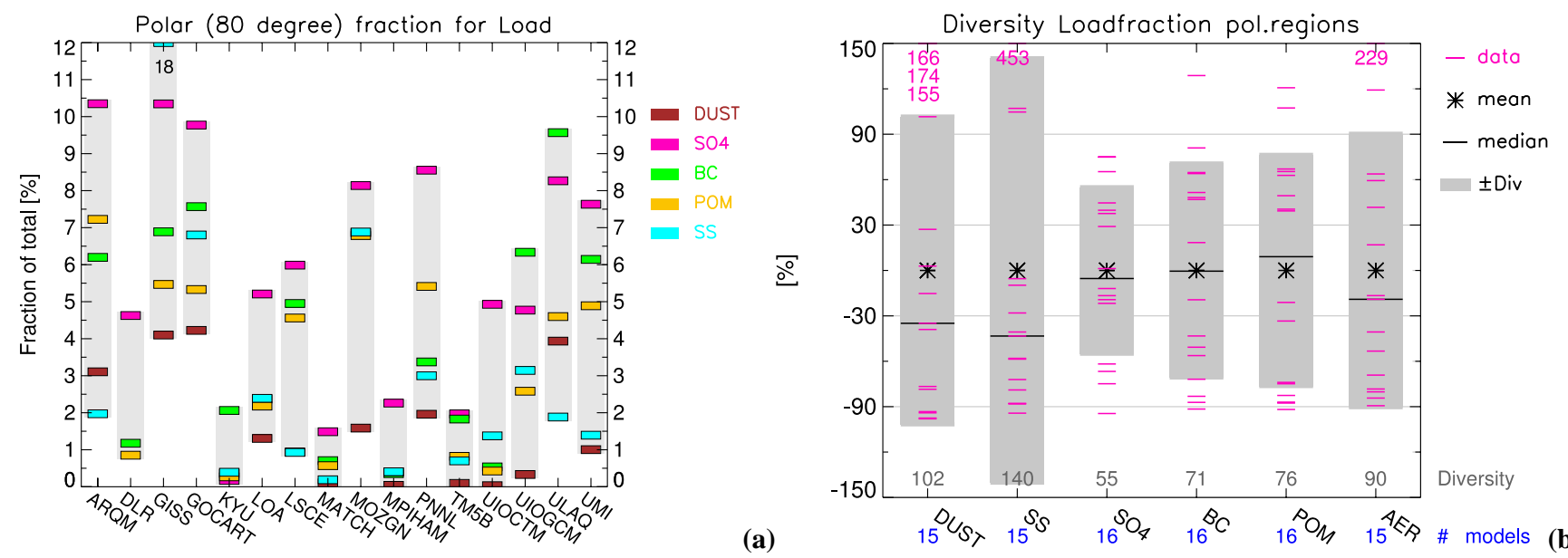

Fig. 12. (a) Global, annual average mass fractions in [\%] of total mass in polar regions (south of $80^{\circ} \mathrm{S}$ and north of $80^{\circ} \mathrm{N}$ ) for all AeroCom models. The ranges of meridional dispersal, i.e., the differences for each model between the species with the largest and the smallest mass fractions in polar regions, respectively, are indicated by the gray shadings. (b) Diversity plot of the annual average aerosol mass fractions in polar regions. For explanations please refer to the caption of Fig. $1 b$.

the lower atmosphere, but we do not diagnose a relationship between dry deposition rate coefficients and vertical dispersivity for the ensemble of AeroCom models, although such a relationship might exist within individual models.

Note that the vertical aerosol dispersivity is also strongly influenced by the intensity of the vertical mixing, which lifts the aerosol to greater heights, at that location of the emission. In addition, the vertical dispersivity depends on the height of emission. This is an issue for volcanic, industrial and also for wildfire emissions. These have sources that are often situated above the planetary boundary layer, leading to elevated residence times. Another sensitive, but not well-constrained process is the representation of aerosol scavenging by ice at low temperatures in the higher atmosphere (e.g., Hendricks et al., 2004; Henning et al., 2004) (see also Table 8).

\subsection{Meridional long-range transport}

A similar diagnostic as for the vertical dispersivity can be performed for the horizontal distribution of aerosol. The mass fractions in polar regions can serve as an indicator for the horizontal dispersivity, i.e., for meridional long-range transport, because polar regions are far from the aerosol sources. We assume here that the differences in the simulated spatial distributions of the sources have no influence on the results. This assumption is justified as our analysis on the AeroCom experiment with unified sources demonstrated (Textor et al., $2006^{3}$ ). Figure 12 shows the mass fractions of aerosols south of $80^{\circ} \mathrm{S}$ and north of $80^{\circ} \mathrm{N}$, and the corresponding diversities of the model results.

The all-models-averages of the mass fractions at the poles in relation to total aerosol are $2.5 \%(\delta=90 \%)$ for AER, $6 \%$ $(\delta=55 \%)$ for $\mathrm{SO}_{4}, 4.2 \%(\delta=71 \%)$ for BC, $3.3 \%(\delta=140 \%)$ for SS, 3.3\% ( $\delta=76 \%$ ) for POM, and $1.5 \%$ ( $\delta=102 \%)$ for DU, respectively. We obtain very high diversities, especially for the "natural" species. In twelve of the 16 models the highest mass fractions at the poles are found for $\mathrm{SO}_{4}$, in three models long-range transport is most efficient for $\mathrm{BC}$, and in one for SS. In twelve models the lowest mass fractions are found for DU, in two for POM, in two for SS. The allmodels-average composition of aerosol particles in polar regions is dominated by SS with $32 \%$ of the total mass, followed by $31 \% \mathrm{DU}, 26 \% \mathrm{SO}_{4}, 9 \% \mathrm{POM}$, and finally $2.1 \%$ $\mathrm{BC}$. We also find little agreement on the ranges of meridional dispersivity in the different models indicated by the gray shadings in Fig. 12, ranging from 1.4\% in MATCH to $14 \%$ in GISS. The models with characteristically low vertical dispersivity (KYU, MATCH, MPI_HAM, TM5) also show fairly weak meridional long-range transport, apart from TM5, where meridional transport is weak, but vertical dispersivity is not. Models with characteristically stronger vertical dispersivity (LOA, LSCE, MOZGN, and UIO_GCM) are not systematically linked with stronger meridional longrange transport. Instead, the latter is more effective in GISS and GOCART than in the other models.

The transport of SS towards polar regions is not very significant in most models, although its most important source regions are in the southern Pacific. This can be attributed to its short residence time. Somewhat higher fractions can however be found in GOCART, MOZGN, and especially in GISS. Long-range transport is most significant for small particles, which have longer residence times. Therefore, the mass fractions in polar regions depend on the contribution of the fine fraction to the total SS mass, and thus on the emitted SS size distribution. This explains the large amount of SS in polar regions found in the GISS model, where the fine mode 
contributes almost $70 \%$ of its total mass, see Fig. 8a. This figure does not however explain the high SS mass fractions in polar regions for the three other models mentioned above, which do not have exceptional contributions of fine mode particles. This can be caused by the quality of our information on the particle sizes (see the discussions in Sect. 8.1) and by the lack of information on the size of the expanded, ambient particles, which are subject to the simulated transport and deposition processes, see Sect. 8.2. Water uptake is particularly weak in MOZGN, see Fig. 9, leading to smaller particles with longer residence times. This could explain the elevated long-range SS-transport towards the poles in this model.

When we compare meridional long-range transport and vertical dispersivity of DU we do not diagnose a correlation (not shown). Hence, we suggest that long-range transport takes place at different heights in the models. The travel distances between the simulated DU sources and polar regions are similar in all models, and we would thus assume more important long-range transports for longer simulated DU residence times. We do not however find such a relationship (not shown) and conclude that the velocities of meridional DU transport differ among the AeroCom models. For "anthropogenic" aerosols we find moderately increased efficiencies of meridional long-range transport in models with slower removal rate coefficients. The (wet) removal rate coefficients of "anthropogenic" aerosols are correlated with the vertical dispersivity, as shown above. Consequently, we also find a positive correlation of vertical dispersivity with the efficiency of meridional long-range transport for the "anthropogenic" aerosols (not shown). We suppose that long-range transport is efficient for fine particles once they have reached the upper free troposphere, where they have extended residence times. This effect stands out of the differences in simulated meridional transport provided by the global models.

\section{Summary and conclusions}

In this paper, we investigated the parameters and processes that govern the simulated aerosol life cycles in sixteen global aerosol models. The diversities among the models' results have been quantified. Aerosol life cycles are influenced by many processes that are highly related. The meteorology of the global model governs horizontal and vertical aerosol transport and provides the relative humidity that influences hygroscopic particle growth. In addition, it controls together with the parameterizations of cloud microphysics the spatial distribution and the strength of precipitation, which in turn affect the aerosol wet deposition efficiency. Boundary layer turbulence and surface cover properties largely control aerosol dry deposition. Furthermore, the aerosol distribution is influenced by the processes and parameters described in the aerosol modules themselves. Based on the present AeroCom data set it was not possible to differentiate whether the transport provided by the global model controls the removal rate coefficients, or if instead contrasting removal processes result in the observed aerosol dispersal. The separation of internal aerosol from transport processes would require the investigation of an inert tracer (e.g., Denning, 1999). The main conclusions from the AeroCom model intercomparison are shortly listed below, see also Table 10.

The AeroCom-models-average results for the properties involved in the life cycle are the following:

- emissions are dominated in mass by SS, followed by $\mathrm{DU}, \mathrm{SO}_{4}, \mathrm{POM}$, and $\mathrm{BC}$,

- burdens from greatest to least are: DU, $\mathrm{SS}, \mathrm{SO}_{4}, \mathrm{POM}$, $\mathrm{BC}$,

- residence times from longest to shortest are: $\mathrm{BC}, \mathrm{POM}$, $\mathrm{DU}, \mathrm{SO}_{4}, \mathrm{SS}$,

- rate coefficients for wet deposition increase with the solubility from $\mathrm{DU}, \mathrm{BC}, \mathrm{POM}$ to $\mathrm{SO}_{4}$ and $\mathrm{SS}$,

- rate coefficients for dry deposition generally correspond to the particle size and are larger for the SS and DU,

- $\mathrm{SO}_{4}, \mathrm{BC}$, and $\mathrm{POM}$ are predominantly removed by wet deposition,

- DU and SS are removed by about two thirds by dry deposition (high model diversity),

- $\mathrm{BC}, \mathrm{POM}$, and $\mathrm{SO}_{4}$ are mainly contained in particles smaller than $1 \mu \mathrm{m}$ in diameter,

- BC, POM, and especially $\mathrm{SO}_{4}$ reach greater heights than the other components.

The established diversities are highest for:

- emissions, particle sizes, residence times, deposition pathways and rate coefficients of SS, DU, AER,

- aerosol composition,

- water content due to hygroscopic growth and ambient (wet) aerosol mass

- vertical dispersivity, mass in the free troposphere,

- long-range transport towards the poles,

- the split between convective and stratiform wet deposition.

The high diversity of the emissions of SS and DU is due to the differences in the parameterizations of their source fluxes and the particle sizes. In addition, the meteorology, the methods employed for nudging and data interpolation, and the model resolutions also play important roles. The high diversity of the deposition pathways and removal rate coefficients of SS and DU are probably related to the disagreement on 
the particle sizes, and possibly also to the application of operator splitting techniques, i.e., the sequential solution of the process equations in numerical models.

The established emission diversities are generally lower for $\mathrm{SO}_{4}, \mathrm{BC}$, and $\mathrm{POM}$ because similar data sets are employed to prescribe their emissions in time and space, and the diversities reflect therefore mainly the differences of these data sets. The diversity of the $\mathrm{SO}_{4}$ sources is mainly caused by different amounts of losses of sulfur-containing precursor gases and by different chemical production rates in the models.

The wet deposition rate coefficients depend on the effects of the scavenging efficiency from the particular parameterizations. These include the calculation of the available water, and the treatment of scavenging by ice, which differ greatly among the models. In addition, the wet deposition rate depends on the temporal and spatial coincidences of aerosols and precipitation.

Aerosol dispersal of a given aerosol component should be more favorable in those models where it has a longer residence time. For $\mathrm{SO}_{4}, \mathrm{BC}$, and $\mathrm{POM}$, we find slightly enhanced dispersals in models with the longer residence times, and a positive correlation between the vertical dispersivity and meridional long-range transport. Once fine aerosols have reached the upper free troposphere, wet scavenging shows a reduced efficiency, and thus meridional long-range transport is more pronounced. However, we do not find such a relationship for SS and DU, nor do we find a systemic correlation between their dispersivities and dry particle sizes within the ensemble of AeroCom models. We suggest that the simulated meridional long-range transport of these species takes place at different heights and at different velocities. The degree of aerosol dispersal is not linked to the model resolution, although it covers a wide range from 304 to 51200 grid points in the horizontal and 18 to 40 layers in the vertical direction, respectively. The diversity of vertical dispersal is smaller than that of meridional dispersal, but they are of similar size for a given species. We therefore conclude that model diversity is propagated from vertical dispersivity to meridional long-range transport.

In some models, a minimum of the aerosol concentration is simulated in the lowest model layer. As potential reasons for this minimum we propose the parameterizations of surface exchange processes by turbulent transport, dry deposition, as well as the use of operator splitting methods to solve the advection-diffusion-processes equation. Further research is needed to clarify the reasons for the simulated surface minimum, especially, because surface concentrations are compared to observational data for model validation.

Sixteen global aerosol models took part in the comparison. The global models are of different architecture, resolution, and include various parameterizations for the subgrid scale processes, some are climatological models. Implemented into these models are aerosol modules of very different complexity. In this study we did not examine the effects of the different model architectures and types. Instead we focused on the investigation of globally and annually averaged quantities to identify disagreements in the simulated aerosol life cycles. The averaging procedure might smooth out divergences resulting from different model formulations. We did not investigate any time dependent quantities, like the amplitude or variability of the simulated annual cycles. We believe, however, that such studies make more sense in the context of a model comparison to observations, which will be discussed in follow-up papers. The data collected in the framework of AeroCom offer many possibilities to examine specific processes, and several surveys are under way. These include comparisons of simulated optical properties and concentrations with ground based and satellite data (Guibert et al., 2006 ${ }^{1}$, Kinne et al., 2006; Schulz et al., 2006 ${ }^{2}$ ), with vertical profiles from LIDAR, aerosol light absorption, and more.

Model intercomparisons have often been criticized for creating peer pressure among the participating groups to convert to all-models-average quantities, but not to improve the scientific knowledge of the investigated system. The establishment of the AeroCom initiative has enhanced interactions within the aerosol scientific community. The publication of the simulation results on the internet has led to the identification of weak components and has provoked the improvement of specific process parameterizations. In addition, model errors could be identified and removed. The diversities established here indicate that aerosol processes in the atmosphere are still not completely understood. Models might give erroneous results under different ambient conditions in climate change scenarios. A process analysis as carried out in this study helps to understand the effects of model-specific parameterizations on macroscopic aerosol features, which can be validated with observations and within climate simulations. Several processes and parameters, which are particularly relevant for aerosol radiative forcing calculations, with high diversities are:

- masses of aerosol in the radiatively active fine mode

- dry aerosol composition

- aerosol water content

- vertical aerosol dispersal.

Consequently the improved representation of these processes and parameters in large-scale aerosol models deserves a high priority in order to reduce the uncertainty of the climatic impact attributed to aerosol.

Acknowledgements. This work was supported by the European Projects PHOENICS (Particles of Human Origin Extinguishing "natural" solar radiation In Climate Systems) and CREATE (Construction, use and delivery of a European aerosol database), and the French space agency CNES (Centre National des Etudes Spatiales). The authors would like to thank the Laboratoire des Sciences du Climat et de l'Environnement, Gif-sur-Yvette, France, and the 
Max-Planck-Institut für Meteorologie, Hamburg, Germany. Work at PNNL was supported largely by the National Aeronautics and Space Administration under Grant NAG5-9531. Pacific Northwest National Laboratory is operated by the U.S. Department of Energy by Battelle Memorial Institute under contract DE-AC06-76RLO 1830. The work of O. Boucher forms part of the Climate Prediction Programme of the UK Department for the Environment, Food and Rural Affairs (DEFRA) under contract PECD 71237.

Edited by: U. Pöschl

\section{References}

Abdul-Razzak, H., Ghan, S. J., and Rivera-Carpio, C.: A parameterization of aerosol activation, Part I: Single aerosol type, J. Geophys. Res., 103, 6123-6132, 1998.

Ackermann, I. J., Hass, H., Ebel, M. M., Binkowski, F. S., and Shankar, U.: Modal Aerosol Dynamics for Europe: Development and first applications, Atmos. Environ., 32, 2981-2999, 1998.

Anderson, B. E. J., Sachse, G. W., Bagwell, D. R., Hudgins, C. H., Blake, D. R., and Blake, N. J.: Aerosols from biomass burning over the tropical South Atlantic region: Distributions and impacts, J. Geophys. Res., 101(D19), 24 117-24 138, doi:10.1029/96JD00717, 1996.

Andreae, M. O. and Merlet, P.: Emission of trace gases and aerosols from biomass burning, Global Biogeochem. Cycles, 15, 955966, 2001

Andres, R. J. and Kasgnoc, A. D.: A time-averaged inventory of subaerial volcanic sulfur emissions, J. Geophys. Res., 103(D19), 25 251-25 261, 1998.

Arakawa, A. and Schubert, W. H.: Interactions of cumulus cloud ensemble with the large-scale environment, J. Atmos. Sci., 31, 671-701, 1974.

Atlas, R. M. and Lucchesi, R.: File Specification for GEOS-DAS Gridded Output, Document No. DAO-1001v4.3, Data Assimilation Office, Goddard Laboratory for Atmospheres, Goddard Space Flight Center, Greenbelt, Maryland, 2000.

Bahreini, R., Jimenez, J. L., Wang, J., Flagan, R. C., Seinfeld, J. H., Jayne, J. T., and Worsnop, D. R.: Aircraft-based aerosol size and composition measurements during ACE-Asia using an Aerodyne aerosol mass spectrometer, J. Geophys. Res. A., 108(D23), 8645, doi:10.1029/2002JD003226, 2003.

Balkanski, Y., Jacob, D. J., and Gardner, G. M.: Transport and Residence Times of Tropospheric Aerosols Inferred from a Global Three-Dimensional Simulation of 210Pb, J. Geophys. Res., 98(D11), 20 573-20 586, 1993.

Balkanski, Y., Schulz, M., Claquin, T., Moulin, C., and Ginoux, P.: Global emissions of mineral aerosol: formulation and validation using satellite imagery, in: Emission of Atmospheric Trace Compounds, edited by: Granier, C., Artaxo, P., and Reeves, C. E., Kluwer, 253-282, 2003.

Barrie, L. A., Yi, Y., Leaitch, W. R., Lohmann, U., Kasibhatla, P., Roelofs, G. J., Wilson, J., McGovern, F., Benkovitz, C., Melieres, M. A., Law, K., Prospero, J., Kritz, M., Bergmann, D., Bridgeman, C., Chin, M., Christensen, J., Easter, R., Feichter, J., Land, C., Jeuken, A., Kjellstrom, E., Koch, D., and Rasch, P.: A comparison of large-scale atmospheric sulphate aerosol models
(COSAM): overview and highlights, Tellus B, 53 (5), 615-645, 2001.

Barth, M. C., Rasch, P. J., Kiehl, J. T., Benkovitz, C. M., and Schwartz, S. E.: Sulfur chemistry in the NCAR CCM: Description, evaluation, features and sensitivity to aqueous chemistry, J. Geophys. Res., 106, 20 311-20 322, 2000.

Bates, T. S., Charlson, R. J., and Gammon, R. H.: Evidence for the climate role of marine biogenic sulphur, Nature, 329, 319-321, 1987.

Bauer, S. E., Balkanski, Y., Schulz, M., Hauglustaine, D. A., and Dentener, F.: Global modeling of heterogeneous chemistry on mineral aerosol surfaces: Influence on tropospheric ozone chemistry and comparison to observations, J. Geophys. Res. A., 109(D2), D02304, doi:10.1029/2003JD003868, 2004.

Bell, N., Koch, D., and Shindell, D. T.: Impacts of chemistryaerosol coupling on tropospheric ozone and sulfate simulations in a General Circulation Model, J. Geophys. Res., 110, D14305, doi:10.1029/2004JD005538, 2005.

Benkovitz, C. M., Berkowitz, C. M., Easter, R. C., Nemesure, S., Wagener, R., and Schwartz, S. E.: Sulfate over the NorthAtlantic and Adjacent Continental Regions - Evaluation for October and November 1986 Using a 3-Dimensional ModelDriven by Observation-Derived Meteorology, J. Geophys. Res. A., 99(D10), 20 725-20 756, 1994.

Benkovitz, C. M., Scholtz, M. T., Pacyna, J., Tarrason, L., Dignon, J., Voldner, E. C., Spiro, P. A., Logan, J. A., and Graedel, T. E.: Global gridded inventories of anthropogenic emissions of sulfur and nitrogen, J. Geophys. Res., 101, 29239-29 253, 1996.

Benkovitz, C. M. and Schwartz, S. E.: Evaluation of Modeled Sulfate and SO2 over North America and Europe for Four Seasonal Months in 1986-87, J. Geophys. Res., 102(D21), 25 305-25338, 1997.

Berge, E.: Coupling of wet scavenging of sulfur to clouds in a numerical weather prediction model, Tellus B, 45, 1-22, 1993.

Berglen, T. F., Berntsen, T. K., Isaksen, I. S. A., and Sundet, J. K.: A global model of the coupled sulfur/oxidant chemistry in the troposphere: The sulfur cycle, J. Geophys. Res., 109, D19310, doi:10.1029/2003JD003948, 2004.

Berntsen, T. and Isaksen, I. S. A.: A global 3-D chemical transport model for the troposphere: 1. Model description and CO and ozone results, J. Geophys. Res., 102(D17), 21 239-21 280, 1997.

Berntsen, T. K., Fuglestvedt, J. S., Myhre, G., Stordal, F., and Berglen, T. F.: Abatement of greenhouse gases: Does location matter?, Climatic Change, in press, 2006.

Binkowski, F. S. and Shankar, U.: The Regional Particulate Matter Model, 1. Model description and preliminary results, J. Geophys. Res., 100, 26 191-26 209, 1995.

Bond, T. C., Streets, D. G., Yarber, K. F., Nelson, S. M., Woo, J.H., and Klimont, Z.: A technology-based global inventory of black and organic carbon emissions from combustion, J. Geophys. Res., 109, D14203, doi:10.1029/2003JD003697, 2004.

Boucher, O. and Anderson, T. L.: GCM assessment of the sensitivity of direct climate forcing by anthropogenic sulfate aerosols to aerosol size and chemistry, J. Geophys. Res., 100, $26117-$ $26134,1995$.

Boucher, O., Pham, M., and Venkataraman, C.: Simulation of the atmospheric sulfur cycle in the Laboratoire de Meteorologie Dynamique General Circulation Model, Model description, model evaluation, and global and European budgets, Note scientifique 
de l'IPSL, 23, 2002.

Brasseur, G. P., Hauglustaine, D. A., Walters, S., Rasch, P. J., Muller, J. F., Granier, C., and Tie, X. X.: MOZART, a global chemical transport model for ozone and related chemical tracers, 1. Model description, J. Geophys. Res. A., 103(D21), 28 265$28289,1998$.

Brinkop, S. and Sausen, R.: A Finite Difference Approximation for Convective Transports Which Maintains Positive Tracer Concentrations, Beitr. Phys. Atmos., 70, 245-248, 1997.

Cakmur, R. V., Miller, R. L., Perlwitz, J., Koch, D., Geogdzhayev, I. V., Ginoux, P., Tegen, I., and Zender, C. S.: Constraining the global dust emission and load by minimizing the difference between the model and observations, J. Geophys. Res., in press, 2006.

Chin, M., Chu, D. A., Levy, R., Remer, L. A., Kaufman, Y. J., Holben, B. N., Eck, T., and Ginoux, P.: Aerosol distribution in the northern hemisphere during ACE-Asia: Results from global model, satellite observations, and sunphotometer measurements, J. Geophys. Res., 109, D23S90, doi:10.1029/2004JD004829, 2004.

Chin, M., Ginoux, P., Kinne, S., Torres, O., Holben, B. N., Duncan, B. N., Martin, R. V., Logan, J. A., Higurashi, A., and Nakajima, T.: Tropospheric aerosol optical thickness from the GOCART model and comparisons with satellite and Sun photometer measurements, J. Atmos. Sci., 59(3), 461-483, 2002.

Chin, M., Rood, R. B., Lin, S.-J., Muller, J. F., and Thompson, A. M.: Atmospheric sulfur cycle in the global model GOCART: Model description and global properties, J. Geophys. Res., 105, 24 671-24 687, 2000.

Chin, M. A., Jacob, D. J., Gardner, G. M., Foremanfowler, M. S., Spiro, P. A., and Savoie, D. L.: A global three-dimensional model of tropospheric sulfate, J. Geophys. Res. A., 101(D13), 18 667-18 690, 1996.

Chuang, C. C., Penner, J. E., Prospero, J. M., Grant, K. E., Rau, G. H., and Kawamoto, K.: Cloud susceptibility and the first aerosol indirect forcing: Sensitivity to black carbon and aerosol concentrations, J. Geophys. Res., 107(D21), 4564, doi:10.1029/2000JD000215, 2002.

Claquin, T., Schulz, M., and Balkanski, Y.: Modeling the mineralogy of atmospheric dust, J. Geophys. Res., 104, 22 243-22 256, 1999.

Claquin, T., Schulz, M., Balkanski, Y., and Boucher, O.: The influence of mineral aerosol properties and column distribution on solar and infrared forcing by dust, Tellus B, 50, 491-505, 1998.

Cooke, W. F., Liousse, C., Cachier, H., and Feichter, J.: Construction of a $1 \times 1$ fossil fuel emission data set for carbonaceous aerosol and implementation and radiative impact in the ECHAM4 model, J. Geophys. Res., 104, 22 137-22 162, 1999.

Cooke, W. F. and Wilson, J. J. N.: A global black carbon aerosol model, J. Geophys. Res. A., 101(D14), 19395-19409, 1996.

Corbett, J. J., Fischbeck, P. S., and Pandis, S. N.: Global nitrogen and sulfur inventories for oceangoing ships, J. Geophys. Res., 104, 3457-3470, 1999.

d'Almeida, G. A.: Atmospheric Aerosols, Deepak Publishing, 561, 1991.

Dana, M. T. and Hales, J. M.: Statistical aspects of the washout of polydisperse aerosols, Atmos. Environ., 10, 45-50, 1976.

Del Genio, A. D. and Yao, M.-S.: Efficient cumulus parameterization for long-term climate studies: The GISS scheme, in:
The Representation of Cumulus Convection in Numeric Models, Amer. Meteorol. Soc., 181-184, 1993.

Del Genio, A. D., Kovari, W., Yao, M.-S., and Jonas, J.: Cumulus microphysics and climate sensitivity, J. Climate, 18, 2376-2387, doi:10.1175/JCLI3413.1, 2005.

Del Genio, A. D., Yao, M.-S., Kovari, W., and Lo, K. K.-W.: A prognostic cloud water parameterization for global climate models, J. Climate, 9, 270-304, doi:10.1175/1520-0442, 1996.

Denning, A. S. M. H., Gurney, K. R., Heimann, M., Law, R. M., Rayner, P. J., Fung, I. Y., Fan, S., Taguchi, S., Friedlingstein, P., Balkanski, Y., Maiss, M., and Levin, I.: Three-dimensional transport and concentration of SF6: A model intercomparison study (Transcom 2), Tellus B, 51, 266-297, 1999.

Dentener, F. J. and Crutzen, P. J.: Reaction of N2O5 on tropospheric aerosols: Impact on the global distributions of $\mathrm{NOx}, \mathrm{O} 3$ and $\mathrm{OH}$, J. Geophys. Res., 98, 7149-7163, 1993.

Dentener, F., Kinne, S., Bond, T., Boucher, O., Cofala, J., Generoso, S., Ginoux, P., Gong, S., Hoelzemann, J. J., Ito, A., Marelli, L., Penner, J., Putaud, J.-P., Textor, C., Schulz, M., van der Werf, G. R., and Wilson, J.: Emissions of primary aerosol and precursor gases for the years 2000 and 1750 prescribed data-sets for AeroCom, Atmos. Chem. Phys. Discuss., 6, 2703-2763, 2006.

Duncan, B. N.,Martin, R. V., Staudt, A. C., Yevich, R., and Logan, J. A.: Interannual and Seasonal Variability of Biomass Burning Emissions Constrained by Satellite Observations, J. Geophys. Res., 108(D2), 4040, doi:10.1029/2002JD002378, 2003.

Easter, R. C., Ghan, S. J., Zhang, Y., Saylor, R. D., Chapman, E., Laulainen, N. S., Abdul-Razzak, H., Leung, L. R., Bian, X., and Zaveri, R. A.: MIRAGE: Model description and evaluation of aerosols and trace gases, J. Geophys. Res., 109, D20210, doi:10.1029/2004JD004571, 2004.

Endresen, Ø., Sørgård, E., Sundet, J. K., Dalsøren, S. B., Isaksen, I. S. A., Berglen, T. F., and Gravir, G.: Emission from international sea transportation and environmental impact, J. Geophys. Res., 108(D17), 4560, doi:10.1029/2002JD002898, 2003.

Erickson, D. J., Merrill, J. T., and Duce, R. A.: Seasonal estimates of global atmospheric sea-salt distributions, J. Geophys. Res., 91, 1067-1072, 1986.

Feichter, J., Kjellstrom, E., Rodhe, H., Dentener, F., Lelieveld, J., and Roelofs, G. J.: Simulation of the tropospheric sulfur cycle in a global climate model, Atmos. Environ., 30(10-11), 16931707, 1996.

Fitzgerald, J. W.: Approximation formulas for the equilibrium size of an aerosol particle as a function of its dry size and composition and the ambient relative humidity, J. Appl. Meteorol., 14, 10441049, 1975.

Fuchs, N. A.: Evaporation and Droplet Growth in Gaseous Media, Pergamon Press, New York, 1959.

Fuchs, N. A.: The Mechanics of Aerosols, Pergamon Press, New York, 1964.

Fuchs, N. A. and Sutugin, A. G.: Highly Dispersed Aerosols, Butterworth-Heinemann, Newton, Mass., USA, 105, 1970.

Ganzeveld, L. and Lelieveld, J.: Dry deposition parametrization in a chemistry general circulation model and its influence on the distribution of reactive trace gases, J. Geophys. Res., 100, 20999 $21012,1995$.

Ganzeveld, L., Lelieveld, J., and Roelofs, G. J.: A dry deposition parameterization for sulfur oxides in a chemistry and general circulation model, J. Geophys. Res., 103(D5), 5679-5694, 1998. 
Generoso, S., Breon, F. M., Balkanski, Y., Boucher, O., and Schulz, M.: Improving the seasonal cycle and interannual variations of biomass burning aerosol sources, Atmos. Chem. Phys., 3, 12111222, 2003.

Gerber, H. E.: Supersaturation and Droplet Sectral Evolution in Fog, J. Atmos. Sci., 48(24), 2569-2588, 1991.

Ghan, S., Laulainen, N., Easter, R., Wagener, R., Nemesure, S., Chapman, E., Zhang, Y., and Leung, R.: Evaluation of aerosol direct radiative forcing in MIRAGE, J. Geophys. Res., 106, 52955316, 2001.

Ghan, S. J., Leung, L. R., Easter, R. C., and Abdul-Razzak, H.: Prediction of droplet number in a general circulation model, $\mathrm{J}$. Geophys. Res., 102, 21 777-21 794, 1997.

Gillette, D.: A wind tunnel simulation of the erosion of soil: Effect of soil texture, sandblasting, wind speed, and soil consolidation on dust production, Atmos. Environ., 12, 1735-1743, 1978.

Ginoux, P., Chin, M., Tegen, I., Prospero, J., Holben, B., Dubovik, O., and Lin, S.-J.: Sources and global distributions of dust aerosols simulated with the GOCART model, J. Geophys. Res., 106, 20 255-20 273, 2001.

Ginoux, P., Prospero, J. M., Torres, O., and Chin, M.: Long-term simulation of global dust distribution with GOCART model: correlation with North Atlantic Oscillation, Environ. Modeling \& Software, 19, 113-128, 2004.

Giorgi, F. and Chameides, W. L.: The rainout parameterization in a photochemical model, J. Geophys. Res., 90, 7872-7880, 1985.

Giorgi, F. and Chameides, W. L.: Rainout lifetimes of highly soluble aerosols and gases as inferred from simulations with a general circulation model, J. Geophys. Res., 91, 14 367-14376, 1986.

Gong, S., Barrie, L. A., and Blanchet, J.-P.: Modeling sea salt aerosols in the atmosphere, 1: Model development, J. Geophys. Res., 102, 3805-3818, 1997.

Gong, S. L.: A parameterization of sea-salt aerosol source function for sub- and super- micron particles, Global Biogeochem. Cycles, 17(4), 1097, doi:1029/2003GB002079, 2003.

Gong, S. L., Barrie, L. A., Blanchet, J.-P., Salzen, K. V., Lohmann, U., Lesins, G., Spacek, L., Zhang, L. M., Girard, E., Lin, H., Leaitch, R., Leighton, H., Chylek, P., and Huang, P.: Canadian Aerosol Module: A size-segregated simulation of atmospheric aerosol processes for climate and air quality models 1. Module development, J. Geophys. Res., 108(D1), 4007, doi:10.1029/2001JD002002, 2003.

Gong, S .L., Barrie, L. A., and Lazare, M.: Canadian Aerosol Module (CAM): A size-segregated simulation of atmospheric aerosol processes for climate and air quality models, 2. Global seasalt aerosol and its budgets, J. Geophys. Res., 107(D24), 4779, doi:10.1029/2001JD002004, 2002.

Grewe, V., Brunner, D., Dameris, M., Grenfell, J. L., Hein, R., Shindell, D., and Staehlin, J.: Origin and variability of upper tropospheric nitrogen oxides and ozone at northern mid-latitudes, Atmos. Environ., 35, 3421-3433, 2001.

Griffin, R. J., Dabdub, D., III, D. R. C., and Seinfeld, J. H.: Estimate of Global Atmospheric Organic Aerosol from Oxidation of Biogenic Hydrocarbons, Geophys. Res. Lett., 26, 2721-2724, 1999.

Grini, A., Myhre, G., Sundet, J. K., and Isaksen, I. S. A.: Modeling the annual cycle of sea salt in the global 3-D model Oslo CTM2: Concentrations, fluxes, and radiative impact, J. Climate, 15, 1717-1730, 2002a.
Grini, A., Myhre, G., Zender, C. S., and Isaksen, I. S. A.: Model simulations of dust sources and transport in the global atmosphere: Effects of soil erodibility and wind speed variability, J. Geophys. Res., 110, D02205, doi:10.1029/2004JD005037, 2005.

Grini, A., Zender, C. S., and Colarco, P. R.: Saltation Sandblasting behavior during mineral dust aerosol production, Geophys. Res. Lett., 29(18), 1868, doi:10.1029/2002GL015248, 2002 b.

Guelle, W., Balkanski, Y. J., Dibb, J. E., Schulz, M., and Dulac, F.: Wet deposition in a global size-dependent aerosol transport model, 2. Influence of the scavenging scheme on $210 \mathrm{~Pb}$ vertical profiles, surface concentrations, and deposition, J. Geophys. Res., 103, 28 875-28 891, 1998a.

Guelle, W., Balkanski, Y. J., Schulz, M., Dulac, F., and Monfray, P.: Wet deposition in a global size-dependent aerosol transport model, 1. Comparison of a 1 year $210 \mathrm{~Pb}$ simulation with ground measurements, J. Geophys. Res., 103, 11 429-11 445, 1998b.

Guelle, W., Balkanski, Y. J., Schulz, M., Marticorena, B., Bergametti, G., Moulin, C., Arimoto, R., and Perry, K. D.: Modeling the atmospheric distribution of mineral aerosol: Comparison with ground measurements and satellite observations for yearly and synoptic timescales over the North Atlantic, J. Geophys. Res., 105, 1997-2012, 2000.

Guenther, A., Hewitt, C., Erickson, D., Fall, R., Geron, C., Graedel, T., Harley, P., Klinger, L., Lerdau, M., McKay, W., Pierce, T., Scholes, B., Steinbrecher, R., Tallamraju, R., Taylor, J., and Zimmerman, P.: A global model of natural volatile organic compound emissions, J. Geophys. Res., 100, 8873-8892, 1995.

Guibert, S., Matthias, V., Schulz, M., Bsenberg, J., Eixmann, R., Mattis, I. ,Pappalardo, G.,Perrone, M. R. ,Spinelli, N., and Vaughan, G.: The vertical distribution of aerosol over Europe Synthesis of one year of EARLINET aerosol lidar measurements and aerosol transport modeling with LMDzT-INCA, Atmos. Environ., 39, 2933-2943, 2005.

Hack, J. J.: Parameterization of moist convection in the National Center for Atmospheric Research community climate model (CCM2), J. Geophys. Res., 99, 5551-5568, 1994.

Hack, J. J.: Sensitivity of the simulated climate to a diagnostic formulation for cloud liquid water, J. Climate, 11, 1497-1515, 1998.

Hänel, G.: The properties of atmospheric aerosol particles as functions of the relative humidity at thermodynamic equilibrium with the surrounding moist air, Adv. Geophys., 19, 73-188, 1976.

Hao, W. M. and Liu, M.-H.: Spatial distribution of tropical biomass burning in 1980 with $5 \times 5$ resolution, Global Biogeochem. $\mathrm{Cy}-$ cles, 8, 495-503, 1994.

Hao, W. M., Liu, M. H., and Crutzen, P. J.: Estimates of annual and regional releases of $\mathrm{CO} 2$ and other trace gases to theatmosphere from fires in the tropics, based on the FAO statistics for the period 1975-80, in: Fire in the tropical biota, Ecosystem processes and global challenges, edited by: Goldammer, J. G., Springer Verlag, New York, USA, 440-462, 1990.

Harrington, D. Y. and Kreidenweis, S. M.: Simulations of sulfate aerosol dynamics-I. Model description, Atmos. Environ., 32, 1691-1700, 1998.

Hasumi, H. and Emori, S.: K-1 coupled GCM (MIROC) description, Center for Climate System Research, University of Tokyo, National Institute for Environmental Studies, Frontier Research Center for Global Change, Tokyo, Japan, 38, 2004.

Hauglustaine, D. A., Brasseur, G. P., Walters, S., Rasch, P. J., 
Muller, J. F., Emmons, L. K., and Carroll, C. A.: MOZART, a global chemical transport model for ozone and related chemical tracers, 2. Model results and evaluation, J. Geophys. Res. A., 103(D21), 28 291-28 335, 1998.

Hauglustaine, D. A., Hourdin, F., Jourdain, L., Filiberti, M.-A., Walters, S., Lamarque, J.-F., and Holland, E. A.: Interactive chemistry in the Laboratoire de Météorologie Dynamique general circulation model: Description and background tropospheric chemistry evaluation, J. Geophys. Res., 109, D04314, doi:10.1029/2003JD003957, 2004.

Hendricks, J., Kärcher, B., Döpelheuer, A., Feichter, J., Lohmann, U., and Baumgardner, D.: Simulating the global atmospheric black carbon cycle: a revisit to the contribution of aircraft emissions, Atmos. Chem. Phys., 4, 2521-2541, 2004.

Henning, S., Bojinski, S., Diehl, K., Ghan, S., Nyeki, S., Weingartner, E., Wurzler, S., and Baltensperger, U.: Aerosol partitioning in natural mixed-phase clouds, Geophys. Res. Lett., 31, L06101, doi:10.1029/2003GL019025, 2004.

Henning, S., Weingartner, E., Schmidt, S., Wendisch, M., Gäggeler, H. W., and Baltensperger, U.: Size-dependent aerosol activation at the high-alpine site Jungfraujoch (3580 m a.s.1.), Tellus B, 54, 82-95, 2002.

Hess, M., Koepke, P., and Schult, I.: Optical Properties of Aerosols and Clouds: The software package OPAC, Bull. Amer. Meteorol. Soc., 79, 831-844, 1998.

Hobbs, P. V., Reid, J. S., Kotchenruther, R. A., Ferek, R. J., and Weiss, R.: Direct radiative forcing by smoke from biomass burning, Science, 275(5307), 1776-1778, 1997.

Horowitz, L. W., Walters, S. M., Mauzerall, D. L., Emmons, L. K., Rasch, P. J., Granier, C., Tie, X., Lamarque, J.-F., Schultz, M. G., and Brasseur, G. P.: A global simulation of tropospheric ozone and related tracers: Description and evaluation of MOZART, version 2, J. Geophys. Res., 108, 4784, doi:10.1029/2002JD002853, 2003.

Hourdin, F. and Armengaud, A.: The use of finite-volume methods for atmospheric advection of trace species, 1. test of various formulations in a general circulation model, Mon. Wea. Rev., 127, 822-837, 1999.

Houweling, S., Dentener, F., and Lelieveld, J.: The impact of nonmethane hydrocarbon compounds on tropospheric photochemistry, J. Geophys. Res., 103, 10673-10 696, 1998.

Iversen, T. and Seland, O.: A scheme for process-tagged SO4 and $\mathrm{BC}$ aerosols in NCAR CCM3: Validation and sensitivity to cloud processes, J. Geophys. Res. A., 107(D24), 4751, doi:10.1029/2001JD000885, 2002.

Jeuken, A. B. M., Veefkind, J. P., Dentener, F., Metzger, S., and Gonzalez, C. R.: Simulation of the aerosol optical depth over Europe for August 1997 and a comparison with observations, J. Geophys. Res. A., 106(D22), 28 295-28 311, 2001.

Jeuken, A.B.M., Siegmund, P.C., Heijboer, L.C., Feichter, J., and Bengtsson, L.: On the potential of assimilating meteorological analyses in a global climate model for the purpose of model validation, J. Geophys. Res., 101(16), 16 939-16 950, 1996.

Kanakidou, M., Seinfeld, J. H., Pandis, S. N., Barnes, I. Dentener, F. J., Facchini, M. C., Van Dingenen, R., Ervens, B., Nenes, A., Nielsen, C. J., Swietlicki, E., Putaud, J. P., Balkanski, Y., Fuzzi, S., Horth, J., Moortgat, G. K., Winterhalter, R., Myhre, C. E. L., Tsigaridis, K., Vignati, E., Stephanou, E. G., and Wilson, J.: Organic aerosol and global climate modelling: a review, Atmos.
Chem. Phys., 5, 1053-1123, 2005.

Kettle, A., Andreae, M. O., Amouroux, D., Andreae, T. W., Bates, T. S., Berresheim, H., Bingemer, H., Boniforti, R., Curran, M. A. J., DiTullio, G. R., Helas, G., Jones, G. B., Keller, M. D., Kiene, R. P., Leck, C., Levasseur, M., Malin, G., Maspero, M., Matrai, P., McTaggart, A. R., Mihalopoulos, N., Nguyen, B. C., Novo, A., Putaud, J. P., Rapsomanikis, S., Roberts, G., Schebeske, G., Sharma, S., Simó, R., Staubes, R., Turner, S., and Uher, G.: A global database of sea surface dimethylsulfide (dms) measurements and a method to predict sea surface dms as a function of latitude, longitude and month, Global Biogeochem. Cycles, 13, 399-444, 1999.

Kettle, A., Andreae, M. O., Amouroux, D., Helas, G., Rapsomanikis, S., Roberts, G., Schebeske, G., Uher, G., Bates, T. S., Berresheim, H., Bingemer, H., Staubes, R., Boniforti, R., Matrai, P., Mihalopoulos, A. R. M., Putaud, B. C. N. P., and Turner, S.: A preliminary global database of sea surface dimethyl sulfide as a function of latitude, longitude, and month, EOS Trans. AGU, Fall Meet. Suppl., 77(46), 417, 1996.

Kettle, A. J. and Andreae, M. O.: Flux of dimethylsulfide from the oceans: A comparison of updated data sets and flux models, J. Geophys. Res., 105, 26 793-26 808, 2000.

Kiehl, J. T. and Gent, P. R.: The Community Climate System Model, Version Two, J. Climate, 17, 3666-3682, 2004.

Kiehl, J. T., Hack, J. J., Bonan, G. B., Boville, B. A., Williamson, D. L., and Rasch, P. J.: The National Center for Atmospheric Research Community Climate Model: CCM3, J. Climate, 11, 1131-1149, 1998.

Kinne, S., Lohmann, U., Feichter, J., Schulz, M., Timmreck, C., Ghan, S., Easter, R., Chin, M., Ginoux, P., Takemura, T., Tegen, I., Koch, D., Herzog, M., Penner, J., Pitari, G., Holben, B., Eck, T., Smirnov, A., Dubovik, O., Slutsker, I., Tanre, D., Torres, O., Mishchenko, M., Geogdzhayev, I., Chu, D.A., and Kaufman, Y.: Monthly averages of aerosol properties: A global comparison among models, satellite data, and AERONET ground data, J. Geophys. Res. A., 108(D20), 4634, doi:10.1029/2001JD001253, 2003.

Kinne, S., Schulz, M., Textor, C., Guibert, S., Bauer, S., Berntsen, T., Berglen, T., Boucher, O., Chin, M., Dentener, F., Diehl, T., Feichter, H., Fillmore, D., Ghan, S., Ginoux, P., Gong, S., Grini, A., Hendricks, J., Horowitz, L., Isaksen, I., Iversen, T., Koch, D., Krol, M., Lauer, A., Lamarque, J. F., Liu, X., Montanaro, V., Myhre, G., Penner, J., Pitari, G., Reddy, S., Seland, O., Stier, P., Takemura, T., and Tie, X.: An AeroCom initial assessment - optical properties in aerosol component modules of global models, Atmos. Chem. Phys., 1815-1834, 2006.

Kirkevåg, A. and Iversen, T.: Global direct radiative forcing by process-parameterized aerosol optical properties, J. Geophys. Res., 107(D20), 4433, 2002.

Kirkevåg, A., Iversen, T., Seland, Ø., and Kristjánsson, J. E.: Revised schemes for aerosol optical parameters and cloud condensation nuclei in CCM-Oslo, in: Institute Report Series No. 28, Department of Geosciences, University of Oslo, Oslo, Norway, 2005.

Kline, J., Huebert, B., Howell, S., Blomquist, B., Zhuang. J., Bertram, T., and Carillo, J.: Aerosol composition and size versus altitude measured from the C-130 during ACE-Asia, J. Geophys. Res. A., 109, D19S08, doi:10.1029/2004JD004540, 2004.

Koch, D.: Transport and direct radiative forcing of carbonaceous 
and sulfate aerosols in the GISS GCM, J. Geophys. Res., 106, 20311-20 322, 2001.

Koch, D. and Hansen, J.: Distant origins of Arctic Black Carbon: A GISS ModelE experiment, J. Geophys. Res., 110, D04204, doi:10.1029/2004JD005296, 2005.

Koch, D., Jacob, D., Tegen, I., Rind, D., and Chin, M.: Tropospheric sulfur simulation and sulfate direct radiative forcing in the Goddard Institute for Space Studies general circulation model, J. Geophys. Res. A., 104(D19), 23 799-23 822, 1999.

Koch, D., Schmidt, G. A., and Field, C.: Sulfur, sea salt and radionuclide aerosols in GISS, ModelE, J. Geophys. Res., in press, 2006.

Koepke, P., Hess, M., Schult, I., and Shettle, E. P.: Global aerosol data set, Max-Planck Institute for Meteorology, 44, 1997.

Krol, M., Houweling, S., Bregman, B., Broek, M. v. d., Segers, A., Velthoven, P. V., Peters, W., Dentener, F., and Bergamaschi, P.: The two-way nested global chemistry-transport zoom model TM5:algorithm and applications, Atmos. Chem. Phys., 5, 417432, 2005.

Kulmala, M., Laaksonen, A., and Pirjola, L.: Parameterizations for sulfuric acid/water nucleation rates, J. Geophys. Res., 103(D7), 8301-8307, 1998.

Lavoue, D., Liousse, C., Cachie, R. H., Stocks, B. J., and Goldammer, J. G.: Modeling of carbonaceous particles emitted by boreal and temperate wildfires at northern latitudes, J. Geophys. Res. A., 105(D22), 26 871-26890, 2000.

Le Treut, H. and Li, Z.-X.: Sensitivity of an atmospheric general circulation model to prescribed SST changes: feedback effects associated with the simulation of cloud optical properties, Clim. Dyn., 5, 175-187, 1991.

Liousse, C., Penner, J. E., Chuang, C., Walton, J. J., Eddleman, H., and Cachier, H.: A global three-dimensional model study of carbonaceous aerosols, J. Geophys. Res. A., 101(D14), 19411$19432,1996$.

Liss, P. S. and Merlivat, L.: Air-sea gas exchange rates: introduction and synthesis, in: The role of air-sea exchange in geochemical cycling, edited by: Buat-Menard, P., D. Reidel Publishing Company, 113-127, 1986.

Liu, H., Jacob, D. J., Bey, I., and Yantosca, R. M.: Constraints from $210 \mathrm{~Pb}$ and $7 \mathrm{Be}$ on wet deposition and transport in a global threedimensional chemical tracer model driven by assimilated meteorological fields, J. Geophys. Res., 106(D11), 12 109-12 128, 2001.

Liu, X. and Penner, J. E.: Effect of Mt. Pinatubo H2SO4/H2O aerosol on ice nucleation in the upper troposphere using a global chemistry and transport model (IMPACT), J. Geophys. Res., 107(D12), doi:10.1029/2001JD000455, 2002.

Lohmann, U.: Possible Aerosol Effects on Ice Clouds via Contact Nucleation, J. Atmos.Sci., 59, 647-656, 2002.

Lohmann, U. and Feichter, J.: Global indirect aerosol effects: a review, Atmos. Chem. Phys. Discuss., 4, 7561-7614, 2004.

Lohmann, U., Feichter, J., Chuag, C. C., and Penner, J. E.: Predicting the number of cloud droplets in the ECHAM GCM, J. Geophys. Res., 104, 9169-9198, 1999.

Lohmann, U. and Kärcher, B.: First interactive simulations of cirrus clouds formed by homogeneous freezing in the ECHAM general circulation model, J. Geophys. Res., 107(D10), doi:10.1029/2001JD000767, 2002.

Lohmann, U., Leaitch, W. R., Barrie, L., Law, K., Yi, Y., Bergmann,
D., Bridgeman, C., Chin, M., Christensen, J., Easter, R., Feichter, J., Jeuken, A., Kjellstrom, E., Koch, D., Rasch, P., and Roelofs, G. J.: Vertical distributions of sulfur species simulated by large scale atmospheric models in COSAM: Comparison with observations, Tellus B, 53(5), 646-672, 2001.

Lohmann, U. and Roeckner, E.: Design and performance of a new cloud microphysics scheme developed for the ECHAM general circulation model, Climate Dynamics, 12, 557-572, 1996.

Marchuk, G. I.: Methods of Numerical Mathematics, Springer Verlag, New York, 316, 1975.

Marticorena, B. and Bergametti, G.: Modeling the atmospheric dust cycle: 1. Design of a soil-derived dust emission scheme, J. Geophys. Res., 100, 16415-16430, 1995.

Meloni, D., Alcide di Sarra, Tatiana Di Iorio, and Fiocco, G.: Influence of the vertical profile of Saharan dust on the visible direct radiative forcing, J. Quant. Spectr. Radiat. Trans., 93, 397-413, 2005.

Metzger, S., Dentener, F., Krol, M., Jeuken, A., and Lelieveld, J.: Gas/aerosol partitioning II: global modeling results, J. Geophys. Res. A., 107(D16), 4313, doi:10.1029/2001JD001103, 2002a.

Metzger, S. M., Dentener, F. J., Lelieveld, J., and Pandis, S. N.: Gas/aerosol partitioning I: a computationally efficient model, J. Geophys. Res., 107(D16), 4312, doi:10.1029/2001JD001102, 2002b.

Miller, R. L., Cakmur, R. V., Perlwitz, J., Koch, D., Schmidt, G. A., Geogdzhayev, I. V., Ginoux, P., Prigent, C., and Tegen, I.: Mineral dust aerosols in the NASA Goddard Institute for Space Sciences ModelE atmospheric general circulation model., J. Geophys. Res., in press, 2006.

Ming, Y., and Russell, L. M.: Predicted hygroscopic growth of sea salt aerosol, J. Geophys. Res., 106, 28 259-28 274, 2001.

Monahan, E. C., Spiel, D. E., and Davidson, K. L.: A model of marine aerosol generation via whitecaps and wave disruption, in: Oceanic Whitecaps and Their Role in Air-Sea Exchange, edited by: Monahan, E. C., Spiel, D. E., and Davidson, K. L., D. Reidel, 167-174, 1986.

Mueller, J.-F.: Geographical distribution and seasonal variation of surface emissions and deposition velocities of atmospheric trace gases, J. Geophys. Res., 97, 3787-3804, 1992.

Mueller, J.-F. and Brasseur, G.: IMAGES: A three-dimensional chemical transport model of the global troposphere, J. Geophys. Res., 100, 16445-16490, 1995.

Myhre, G., Berntsen, T. K., Haywood, J. M., Sundet, J. K., Holben, B. N., Johnsrud, M., and Stordal, F.: Modelling the solar radiative impact of aerosols from biomass burning during the Southern African Regional Science Initiative (SAFARI-2000) experiment, J. Geophys. Res., 108, 8501, doi:10.1029/2002JD002313, 2003.

Nakicenovic, N., Alcamo, J., Davis, G., Vries, B. d., Fenhann, J., Gaffin, S., Gregory, K., Grübler, A., Jung, T. Y., Kram, T., Rovere, E. L. L., Michaelis, L., Mori, S., Morita, T., Pepper, W., Pitcher, H., Price, L., Riahi, K., Roehrl, A., Rogner, H.-H., Sankovski, A., Schlesinger, M., Shukla, P., Smith, S., Swart, R., Rooijen, S. v., Victor, N., and Dadi, Z.: Emissions Scenarios, A Special Report of Working Group III of the Intergovernmental Panel on Climate Change, Cambridge University Press, Cambridge, United Kingdom and New York, NY, USA, 599, 2000.

Nightingale, P., Malin, G., Law, C., Watson, A., Liss, P., Liddicoat, M., Boutin, J., and Upstill-Goddard, R.: In situ evaluation of air-sea exchange parameterizations using novel conservative and 
volatile tracers, Global Biogeochem. Cycles, 14, 373-387, 2000.

Nordeng, T. E.: Extended version of the convective parametrization scheme at ECMWF and their impact on the mean and transient activity of the model in the tropics, ECMWF Tech. Report 206, 1994

Nozawa, T. and Kurokawa, J.: Historical and future emissions of sulfur dioxide and black carbon for global and regional climate change studies, CGER-Report, CGER/NIES, Tsukuba, Japan, in press, 2006.

Numaguti, A., Takahashi, M., Nakajima, T., and Sumi, A.: Developmentof an atmospheric general circulation model, in: Climate SystemDynamics and Modeling, edited by: Matsuno, T., Cent. for Clim. Syst. Res., Univ. of Tokyo, Tokyo, 1-27, 1995.

Olivier, J. G. J., Bouwman, A. F., Maas, C. W. M. V. d., Berdowski, J. J. M., Veldt, C., Bloss, J. P. J., Vesschedijk, A. J. H., Zandveldt, P. Y. J., and Haverlag, J. L.: Description of EDGAR Version 2.0: A set of global emission inventories of greenhouse gases and ozone-depleting substances for all antrhopogenic and most natural sources on a per country basis and on a $1 \times 1$ grid, pp. RIVM report 771060002/ TNO-MEP report R96/119, Rijkinstituut voor Volksgezondheid en Milieu, Bilthoven, Netherlands, 1996.

Olivier, J. G. J., Berdowski, J. J. M., Peters, J. A. H. W., Bakker, J., Visschedijk, A. J. H., and Bloos, J. P. J.: Applications of EDGAR including a description of EDGAR V3.0: reference database with trend data for 1970-1995, NRP Report, 410200 051, RIVM, Bilthoven, The Netherlands, 2002.

Penner, J. E., Andreae, M., Annegarn, H., Barrie, L., Feichter, J., Hegg, D., Jayaraman, A., Leaitch, R., Murphy, D., Nganga, J., Pitari, G., et al.: Aerosols, their Direct and Indirect Effects, in: Climate Change 2001: The Scientific Basis, Contribution of Working Group I to the Third Assessment Report of the Intergovernmental Panel on Climate Change (IPCC), Chapter 5, edited by: Houghton, J. T., Ding, Y., Griggs, D. J., Noguer, M., Linden, P. J. v. d., and Xiaosu, D., Cambridge University Press, Cambridge, 289-348, 2001.

Penner, J. E., Atherton, C. A., and Graedel, T. .: Global emissions and models of photochemically active compounds, in: Global Atmospheric-Biospheric Chemistry, edited by: Prinn, R., Plenum Publishing, NY, 223-248, 1994.

Penner, J. E., Eddleman, H., and Novakov, T.: Towards the development of a global inventory of black carbon emissions, Atmos. Environ., 27A, 1277-1295, 1993.

Penner, J. E., Zhang, S. Y., Chin, M., Chuang, C. C., Feichter, J., Feng, Y., Geogdzhayev, I. V., Ginoux, P., Herzog, M., Higurashi, A., Koch, D., Land, C., Lohmann, U., Mishchenko, M., Nakajima, T., Pitari, G., Soden, B., Tegen, I., and Stowe, L.: A comparison of model- and satellite-derived aerosol optical depth and reflectivity, J. Atmos. Sci., 59(3), 441-460, 2002.

Pham, M., Muller, J.-F. M., Brasseur, G. P., Granier, C., and Megie, G. M.: A three-dimensional study of the tropospheric sulfur cycle, J. Geophys. Res., 100(D12), 26 061-26 092, 1995.

Pitari, G., Mancini, E., Rizi, V., and Shindell, D. T.: Impact of future climate and emissions changes on stratospheric aerosols and ozone, J. Atmos. Sci., 59, 414-440, 2002.

Pitari, G., Rizi, V., Ricciardulli, L., and Visconti, G.: High-speed civil transport impact: Role of sulfate, nitric acid trihydrate, and ice aerosol studied with a two-dimensional model including aerosol physics, J. Geophys. Res., 98, 23 141-23 164., 1993.

Pruppacher, H. R. and Klett, J. D.: Microphysics of Clouds and Pre- cipitation, second edition, Kluwer Academic Publishers, 1997.

Pueschel, R. F., Blake, D. F., Snetsinger, K. G., Hansen, A. D. A., Verma, S., and Kato, K.: Black Carbon (soot) aerosol in the lower stratosphere and upper troposhere, Geophys. Res. Lett., 19, 16 1659-16 1662, 1992.

Radke, L. F., Hegg, D. A., Lyons, J. H., Brock, C. A., Hobbs, P. V., Weiss, R., and Rasmussen, R.: Airborne measurements on smokes from biomass burning, in: Aerosols and Climate, edited by: Hobbs, P. V. and McCormick, M. P., A Deepak Publishing, Hampton, VA, 411-422, 1988.

Rasch, P. J., Collins, W. D., and Eaton, B. E.: Understanding the INDOEX aerosol distributions with an aerosol assimilation, J. Geophys. Res., 106, 7337-7355, 2001.

Rasch, P. J., Feichter, J., Law, K., Mahowald, N., Penner, J., Benkovitz, C., Genthon, C., Giannakopoulos, C., Kasibhatla, P., Koch, D., Levy, H., Maki, T., Prather, M., Roberts, D.L., Roelofs, G.-J., Stevenson, D., Stockwell, Z., Taguchi, S., M., K., Chipperfield, M., Baldocchi, D., McMurry, P., Barrie, L., Balkanski, Y., Chatfield, R., Kjellström, E., Lawrence, M., Lee, H. N., Lelieveld, J., Noone, K. J., Seinfeld, J., Stenchikov, G., Schwartz, S., Walcek, C., and Williamson, D.L.: A comparison of scavenging and deposition processes in global models: results from the WCRP Cambridge Workshop of 1995, Tellus B, 52, 1025-1056, 2000

Rasch, P. J. and Kristjansson, J. E.: A comparison of the CCM3 model climate using diagnosed and predicted condensate parameterizations, J. Climate, 11, 1587-614, 1998.

Rasch, P. J., Mahowald, N. M., and Eaton, B. E.: Representations of transport, convection, and the hydrological cycle in chemical transport models: Implications for the modeling of short-lived and soluble species, J. Geophys. Res., 102, 28 127-28 138, 1997.

Reddy, M. S. and Boucher, O.: Global carbonaceous aerosol transport and assessment of radiative effects in the LMDZ GCM, J. Geophys. Res., 109(D14), D14202, doi:10.1029/2003JD004048, 2004.

Reddy, M. S., Boucher, O., Bellouin, N., Schulz, M., Balkanski, Y., Dufresne, J.-L., and Pham, M.: Estimates of global multicomponent aerosol optical depth and radiative forcing perturbation in the Laboratoire de Meteorologie Dynamique general circulation model, J. Geophys. Res., 110, D10S16, doi:10.1029/2004JD004757, 2005.

Roeckner, E., Arpe, K., Bengtsson, L., Christoph, M., Claussen, M., Dümenil, L., Esch, M., Giorgetta, M., Schlese, U., and Schulzweida, U.: The atmospheric general circulation model (ECHAM-4): Model description and simulation of the presentday climate, Max-Planck-Institut für Meteorologie, Hamburg, 1996.

Roeckner, E., Brokopf, R., Esch, M., Giorgetta, M., Hagemann, S., Kornblueh, L., Manzini, E., Schlese, U., and Schulzweida, U.: The atmospheric general circulation model ECHAM5 Part II: Sensitivity of simulated climate to horizontal and vertical resolution, Max-Planck-Institute for Meteorology, Report No. 354 , Hamburg, Germany, 2004.

Roeckner, E., Bäuml, G., Bonaventura, L., Brokopf, R., Esch, M., Giorgetta, M., Hagemann, S., Kirchner, I., Kornblueh, L., Manzini, E., Rhodin, A., Schlese, U., Schulzweida, U., and Tompkins, A.: The atmospheric general circulation model ECHAM5, Part I: Model description, Max-Planck-Institute for Meteorology, Report No. 349, Hamburg, Germany, 2003. 
Roelofs, G. J., Kasibhatla, P., Barrie, L., Bergmann, D., Bridgeman, C., Chin, M., Christensen, J., Easter, R., Feichter, J., Jeuken, A., Kjellstrom, E., Koch, D., Land, C., Lohmann, U., and Rasch, P.: Analysis of regional budgets of sulfur species modeled for the COSAM exercise, Tellus B, 53(5), 673-694, 2001.

Roelofs, G.-J. and Lelieveld, J.: Distribution and budget of O3 in the troposphere calculated with a chemistry-general circulation model, J. Geophys. Res., 100, 20 983-20 998, 1995.

Rossow, W. B., Garder, B. L., Lu, P. J., and Walker, A. W.: Satellite cloud climatology project (ISCCP) documentation on cloud data, in: Tech. Doc. WMO/TD-266, WMO, 78, 1987.

Rotman, D. A., Atherton, C. S., Bergmann, D. J., Cameron-Smith, P. J., Chuang, C. C., Connell, P. S., Dignon, J. E., Franz, A., Grant, K. E., Kinnison, D. E., Molenkamp, C. R., Proctor, D. D., and Tannahill, J. R.: IMPACT, the LLNL 3-D global atmospheric chemical transport model for the combined troposphere and stratosphere: Model description and analysis of ozone and other trace gases, J. Geophys. Res., 109, D04303, doi:10.1029/2002JD003155, 2004.

Sadourny, R. and Laval, K.: January and July performance of the LMD general circulation model, in: New Perspectives in Climate Modelling, edited by: Berger, A. and Nicolis, C., Elsevier Sci., New York, 173-198, 1984.

Schmidt, G. A., Ruedy, R., Hansen, J. E., Aleinov, I., Bell, N., Bauer, M., Bauer, S., Cairns, B., Canuto, V., Cheng, Y., Del Genio, A., Faluvegi, G., Friend, A. D., Hall, T. M., Hu, Y., Kelley, M., Kiang, N. Y., Koch, D., Lacis, A. A., Lerner, J., Lo, K. K., Miller, R. L., Nazarenko, L., Oinas, V., Perlwitz, Ja., Perlwitz, Ju., Rind, D., Romanou, A., Russell, G. L., Sato, Mki., Shindell, D. T., Stone, P. H., Sun, S., Tausnev, N., Thresher, D., and Yao, M.-S.::Present day atmospheric simulations using GISS ModelE: Comparison to in-situ, satellite and reanalysis data, J. Climate, 19, 153-192, 2006.

Schubert, S. D., Rood, R. B., and Pfaendtner, J.: An assimilated data set for earth science applications, Bull. Am. Meteorol. Soc., 74, 2331-2342, 1993.

Schulz, M., Balkanski, Y. J., Guelle, W., and Dulac, F.: Role of aerosol size distribution and source location in a threedimensional simulation of a Saharan dust episode tested against satellite-derived optical thickness, J. Geophys. Res. A., 103(D9), 10 579-10 592, 1998.

Schulz, M., De Leeuw, G., and Balkanski, Y.: Sea-salt aerosol source functions and emissions, in: Emission of Atmospheric Trace Compounds, edited by: Granier, C., Artaxo, P., and Reeves, C. E., Kluwer, 333-359, 2004.

Seinfeld, J. H. and Pandis, S. N.: Atmospheric chemistry and physics, Wiley-Interscience publication, 1360, 1997.

Slinn, W. G. N.: Precipitation scavenging, in: Atmospheric Science and Power Production, edited by: Randerson, D., Tech. Inf. Cent., Off. Of Sci. and Tech. Inf., U.S. Dep. Of Energy, Doc. DOE/TIC-27601, Washington, D.C., 466-532 1984.

Smith, M. H. and Harrison, N. M.: The sea spray generation function, J. Aerosol Sci., 29, S189-S190, 1998.

Spiro, P. A., Jacob, D. J., and Logan, J. A.: Global inventory of sulfur emissions with $1^{\circ} \times 1^{\circ}$ resolution, J. Geophys. Res., 97, 6023-6036, 1992.

Stier, P., Feichter, J., Kinne, S., Kloster, S., Vignati, E., Wilson, J., Ganzeveld, L., Tegen, I., Werner, M., Balkanski, Y., Schulz, M., and Boucher, O.: The aerosol-climate model ECHAM5-HAM,
Atmos. Chem. Phys., 5, 1125-1156, 2005.

Streets, D. G., Bond, T. C., Carmichael, G. R., Fernandes, S. D., Fu, Q., He, D., Klimont, D., Nelson, S. M., Tsai, N. Y., Wang, M. Q., Woo, J. H., and Yarber, K. F.: An inventory of gaseous and primary aerosol emissions in Asia in the year 2000, J. Geophys. Res., 108(D21), 8809, doi:10.1029/2002JD003093, 2003.

Sudo, K., Takahashi, M., and Akimoto, J. K.: CHASER: A global chemical model of the troposphere: 1. Model description, J. Geophys. Res., 107(D17), 4339, doi:10.1029/2001JD001113, 2002.

Takemura, T., Nozawa, T. T., Emori, S., Nakajima, T. Y., and Nakajima, T.: Simulation of climate response to aerosol direct and indirect effects with aerosol transport-radiation model, J. Geophys. Res., 110, D02202, doi:10.1029/2004JD005029, 2005.

Takemura, T., Nakajima, T., Dubovik, O., Holben, B. N., and Kinne, S.: Single-scattering albedo and radiative forcing of various aerosol species with a global three-dimensional model, J. Climate, 15(4), 333-352, 2002.

Takemura, T., Okamoto, H., Maruyama, Y., Numaguti, A., Higurashi, A., and Nakajima, T.: Global three-dimensional simulation of aerosoloptical thickness distribution of various origins, J. Geophys. Res., 105, 17 853-17 873, 2000.

Tang, I. N.: Chemical and size effects of hygroscopic aerosols on light scattering coefficients, J. Geophys. Res., 101(D14), 19245$19250,1996$.

Tang, I. N. and Munkelwitz, H. R.: Determination of vapor pressure from droplet evaporation kinetics, J. Colloid Interface Sci., 141, 109-118, 1991.

Tang, I. N. and Munkelwitz, H. R.: Water activities, densities, and refractive indices of aqueous sulfates and sodium nitrate droplets of atmospheric importance, J. Geophys. Res., 99(D9), 18 801$18808,1994$.

Tang, I. N., Tridico, A. C., and Fung, K. H.: Thermodynamic and optical properties of sea salt aerosols, J. Geophys. Res., 102, 23 269-23 275, 1997.

Tang, I. N., Wong, W. T., and Munkelwitz, H. R.: The relative importance of atmospheric sulfates and nitrates in visibility reduction, Atmos. Environ., 15, 2463-2471, 1981.

Tegen, I., Harrison, S. P., Kohfeld, K., Prentice, I. C., Coe, M., and Heimann, M.: Impact of vegetation and preferential source areas on global dust aerosol: Results from a model study, J. Geophys. Res. A., 107(D21), 4576, doi:10.1029/2001JD000963, 2002.

Tie, X., Brasseur, G., Emmons, L., Horowitz, L., and Kinnison, D.: Effects of aerosols on tropospheric oxidants: A global model study, J. Geophys. Res., 106, 22 931-22 964, 2001.

Tie, X. X., Madronich, S., Walters, S., Edwards, D. P., Ginoux, P., Mahowald, N., Zhang, R. Y., Lou, C., and Brasseur, G.: Assessment of the global impact of aerosols on tropospheric oxidants, J. Geophys. Res. A., 110, D03204, doi:10.1029/2004JD005359, 2005.

Tiedtke, M.: A comprehensive mass flux scheme for cumulus parameterization in large-scale models, Mon. Wea. Rev., 117, 1779-1800, 1989.

Timmreck, C. and Schulz, M.: Significant dust simulation differences in nudged and climatological operation mode of the AGCM ECHAM, J. Geophys. Res., 109, D13202, doi:10.1029/2003JD004381, 2004.

Tompkins, A. M.: A Prognostic Parameterization for the SubgridScale Variability of Water Vapor and CLouds in Large-Scale Models and Its Use to Diagnose Cloud Cover, J. Atmos. Sci., 
59(12), 1917-1942, 2002.

Van der Werf, G. R., Randerson, J. T., Collatz, G. J., and Giglio, L.: Carbon emissions from fires in tropical and subtropical ecosystems, Global Change Biology, 9, 547-562, 2003.

Vehkamäki, H., Kulmala, M., Napari, I., Lehtinen, K. E. J., Timmreck, C., Noppel, M., and Laaksonen, A.: An improved parameterization for sulfuric acid-water nucleation rates for tropospheric and stratospheric conditions, J. Geophys. Res., 107(D22), 46224631, doi:10.1029/2002JD002184, 2002.

Vignati, E., Wilson, J., and Stier, P.: M7: An efficient sizeresolved aerosol microphysics module for large-scale aerosol transport models, J. Geophys. Res., 109(D22), D22202, doi:10.1029/2003JD004485, 2004.

von Salzen, K. and Schlunzen, K. H.: A prognostic physicochemical model of secondary and marine inorganic multicomponent aerosols II, Model tests, Atmos. Environ., 33(10), 15431552, 1999.

Wanninkhof, R.: Relationship Between Wind Speed and Gas Exchange Over the Ocean, J. Geophys. Res., 97, 7373-7382, 1992.

Wesely, M. L.: Parameterization of surface resistance to gaseous dry deposition in regional-scale numerical models, Atmos. Environ., 23, 1293-1304, 1989.
$\mathrm{Xu}, \mathrm{K} . \mathrm{M}$. and Krueger, S. K.: Evaluation od cloudiness parameterizations using a cumulus ensemble model, Mon. Wea. Rev., 119, 342-367, 1991.

Zender, C. S., Bian, H., and Newman, D.: The mineral Dust Entrainment And Deposition (DEAD) model: Description and 1990's dust climatology, J. Geophys. Res., 108(D14), 4416, doi:10.1029/2002JD002775, 2003.

Zhang, G. J. and McFarlane, N. A.: Role of convective-scale momentum transport in climate simulation, J. Geophys. Res., 100, 1417-1426, 1995a.

Zhang, G. J., and McFarlane, N. A.: Sensitivity of Climate Simulations to the Parameterization of Cumulus Convection in the Canadian Climate Centre General Circulation Model, Atmosphere-Ocean, 33, 407-446, 1995 b.

Zhang, L., Gong, S.-L., Padro, J., and Barrie, L.: A Size-segregated Particle Dry Deposition Scheme for an Atmospheric Aerosol Module, Atmos. Environ., 35(3), 549-560, 2001.

Zhang, M. H., Lin, W. Y., Bretherton, C., Hack, J. J., and Rasch, P. J.: A modified formulation of fractional stratiform condensation rate in the NCAR Community Atmospheric Model CAM2, J. Geophys. Res., 108(D1), 4035, doi:10.1029/2002JD002523, 2003. 